\title{
Robust Likelihood Estimation of Dynamic Panel Data Models*
}

\author{
Javier Alvarez ${ }^{1} \quad$ Manuel Arellano ${ }^{2}$
}

This version: December 2004

\begin{abstract}
We develop likelihood-based estimators for autoregressive panel data models that are consistent in the presence of time series heteroskedasticity. Bias corrected conditional score estimators, random effects maximum likelihood (RML) in levels and first differences, and estimators that impose mean stationarity are considered for $\operatorname{AR}(p)$ models with individual effects. We investigate identification under unit roots, and show that RML in levels may achieve substantial efficiency gains relative to estimators from data in differences. In an empirical application, we find evidence against unit roots in individual earnings processes from the PSID and the Spanish section of the European Panel.
\end{abstract}

\footnotetext{
${ }^{1}$ Universidad de Alicante, Departamento de Fundamentos del Análisis Económico, 03071 Alicante, Spain (e-mail: alvarez@merlin.fae.ua.es).

${ }^{2}$ CEMFI, Casado del Alisal 5, 28014 Madrid, Spain. (e-mail: arellano@cemfi.es).
}

*We are grateful to Olympia Bover, Enrique Sentana, and seminar audiences at Alicante, CEMFI, Princeton, University College London, and Yale for helpful comments, and to Laura Hospido and Gema Zamarro for very able research assistance. 


\section{Introduction}

The generalized method of moments (GMM) is routinely employed in the estimation of autoregressive models from short panels, because it provides simple estimates that are fixed- $T$ consistent and optimally enforce the model's restrictions on the data covariance matrix. Yet they are known to frequently exhibit poor properties in finite samples and may be asymptotically biased if $T$ is not treated as fixed.

There are also available in the literature fixed- $T$ consistent maximum likelihood methods that are likely to have very different properties to GMM in finite samples and double asymptotics. This category includes random effects estimators of the type considered by Blundell and Smith (1991) and Alvarez and Arellano (2003), the conditional likelihood estimator in Lancaster (2002), and the estimators for first-differenced data in Hsiao, Pesaran, and Tahmiscioglu (2002). However, the existing likelihood-based estimators require that the error variances remain constant through time for fixed- $T$ consistency. Lack of robustness to time series heteroskedasticity is an important limitation because the dispersion of the cross-sectional distribution of errors at each period may differ not only due to nonstationarity at the individual level but also as a result of aggregate effects.

In this paper we develop likelihood-based estimators of autoregressive models that are robust in the sense that remain consistent under the same assumptions as standard panel GMM procedures. ${ }^{1}$ From a GMM perspective, likelihood-based estimation can be motivated as a way of reducing the number of moments available for estimation, and hence the extent of bias in second-order or double asymptotics. Our methods are robust in the sense used in Gourieroux, Monfort, and Trognon (1984) of providing consistent estimates of the conditional mean parameters when the chosen likelihood function does not necessarily contain the true distribution.

The paper is organized as follows. Section 2 presents the model and a discussion of the assumptions. Section 3 explains how to obtain fixed- $T$ consistent estimates of $\operatorname{AR}(p)$ coefficients from bias-corrected first-order conditions of a heteroskedastic within-groups likelihood (BCS).

Section 4 presents ML estimates from a likelihood averaged with respect to normally distributed effects and initial observations (RML). We show that such an

\footnotetext{
${ }^{1}$ Cf. Holtz-Eakin, Newey, and Rosen (1988), Arellano and Bond (1991), Arellano and Bover (1995), and Ahn and Schmidt (1995).
} 
averaging leads to a modified within-groups criterion that balances off the within and between biases. The modification term, which depends on the data in levels, may lead to substantial efficiency gains relative to estimators from differenced data alone, and is crucial for identification in very short panels. Heteroskedastic RML is our recommended likelihood-based method. It is computationally straightforward and can be easily extended to unbalanced and multivariate panels.

Section 5 presents RML estimates from data in differences, and Section 6 discusses conditional and marginal ML estimation under stationarity in mean. Interestingly, we show that the random effects likelihood for the differenced data coincides with the likelihood conditioned on the estimated effects under mean stationarity, so that this restriction is immaterial to the data in differences when homoskedasticity is not imposed.

Section 7 discusses the possibility of identification failure for a first-order process with an unit root, in view that in a three-wave panel a random walk without heterogeneous drift is known to be underidentified. We show that in a four-wave panel there is local identification but not global identification under heteroskedasticity, and global identification but first-order underidentification under homoskedasticity. In panels with more than four waves, we find that the autoregressive coefficient is globally identified unless the error variances change with a constant rate of growth.

Section 8 reports numerical calculations of the asymptotic variances of BCS and RML estimators in differences relative to RML in levels, calculated under the assumption of normality. In Section 9 we present estimates of first- and second-order autoregressive equations for individual labour income using data from the PSID and the Spanish section of the European Panel, and find evidence against unit roots in earnings. The PSID result is in contrast with the income processes that impose a unit root, often employed in the empirical literatures on consumption and labour supply (e.g. Hall and Mishkin, 1982; Abowd and Card, 1989, or Meghir and Pistaferri, 2004). Our result is unaffected by adding moving average components to the specification of the earnings process.

Finally, Section 10 contains some concluding remarks on double asymptotic properties. Proofs and technical material are in the Appendix. 


\section{Model and Assumptions}

We consider an autoregressive model for panel data given by

$$
y_{i t}=\alpha_{1} y_{i(t-1)}+\ldots+\alpha_{p} y_{i(t-p)}+\eta_{i}+v_{i t} \quad(t=1, \ldots, T ; i=1, \ldots, N) .
$$

The variables $\left(y_{i(1-p)}, \ldots, y_{i 0}, \ldots, y_{i T}\right)$ are observed but $\eta_{i}$ is an unobservable individual effect. The $p \times 1$ vector of initial observations is denoted as $y_{i}^{0}=$ $\left(y_{i(1-p)}, \ldots, y_{i 0}\right)^{\prime}{ }^{2}$ We abstract from additive aggregate effects by regarding $y_{i t}$ as a deviation from a time effect. It is convenient to introduce the notation $x_{i t}=\left(y_{i(t-1)}, \ldots, y_{i(t-p)}\right)^{\prime}, \alpha=\left(\alpha_{1}, \ldots, \alpha_{p}\right)^{\prime}$, and write the model in the form:

$$
y_{i}=X_{i} \alpha+\eta_{i} \iota+v_{i}
$$

where $y_{i}=\left(y_{i 1}, \ldots, y_{i T}\right)^{\prime}, X_{i}=\left(x_{i 1}, \ldots, x_{i T}\right)^{\prime}, \iota$ is a $T \times 1$ vector of ones, and $v_{i}=\left(v_{i 1}, \ldots, v_{i T}\right)^{\prime}$.

The following assumption will be maintained throughout:

Assumption $A:\left\{\eta_{i}, y_{i}^{0}, y_{i 1}, \ldots, y_{i T}\right\}_{i=1}^{N}$ is a random sample from a well defined joint distribution with finite fourth-order moments that satisfies

$$
E\left(v_{i t} \mid \eta_{i}, y_{i}^{0}, y_{i 1}, \ldots, y_{i(t-1)}\right)=0 \quad(t=1, \ldots, T) .
$$

This is our core condition in the sense that we wish to consider estimators that are consistent and asymptotically normal for fixed $T$ and large $N$ under Assumption $A$.

Note that neither time series or conditional heteroskedasticity are assumed. That is, the unconditional variances of the errors, denoted as

$$
E\left(v_{i t}^{2}\right)=\sigma_{t}^{2},
$$

are allowed to change with $t$ and to differ from the conditional variances

$$
E\left(v_{i t}^{2} \mid \eta_{i}, y_{i}^{0}, y_{i 1}, \ldots, y_{i(t-1)}\right) \text {. }
$$

Time series homoskedasticity is a particularly restrictive assumption in the context of short micropanels, both because estimators that enforce homoskedasticity are inconsistent when the assumption fails, and because it can be easily violated if aggregate effects are present in the conditional variance of the process.

\footnotetext{
${ }^{2}$ We assume that $y_{i}^{0}$ is observed for notational convenience, so that the actual number of waves in the data is $T^{o}=T+p$.
} 
Also note that under stability of the process, ${ }^{3}$ we do not assume stationarity in mean. Let the covariance matrix of $\left(\eta_{i}, y_{i}^{0}\right)$ be denoted as

$$
\operatorname{Var}\left(\begin{array}{c}
\eta_{i} \\
y_{i}^{0}
\end{array}\right)=\left(\begin{array}{cc}
\sigma_{\eta}^{2} & \gamma_{\eta 0} \\
\gamma_{0 \eta} & \Gamma_{00}
\end{array}\right)
$$

For example, when $p=1$ (so that $\alpha=\alpha_{1}, y_{i}^{0}=y_{i 0}$, and $\left.\Gamma_{00}=\gamma_{00}\right)$ model $(2.1)$ can be written as

$$
y_{i t}=\left(1+\alpha+\ldots+\alpha^{t-1}\right) \eta_{i}+\alpha^{t} y_{i 0}+\left(v_{i t}+\alpha v_{i(t-1)}+\ldots+\alpha^{t-1} v_{i 1}\right) .
$$

Thus, when $|\alpha|<1$, for large $t E\left(y_{i t} \mid \eta_{i}\right)$ tends to the steady state mean $\mu_{i}=$ $\eta_{i} /(1-\alpha)$. If the process started in the distant past we would have

$$
y_{i 0}=\frac{\eta_{i}}{(1-\alpha)}+\sum_{j=0}^{\infty} \alpha^{j} v_{i(-j)}
$$

implying $\gamma_{\eta 0}=\sigma_{\eta}^{2} /(1-\alpha)$ and $\gamma_{00}=\sigma_{\eta}^{2} /(1-\alpha)^{2}+\sum_{j=0}^{\infty} \alpha^{2 j} \sigma_{-j}^{2} \cdot{ }^{4}$ However, here $\gamma_{\eta 0}$ and $\gamma_{00}$ are treated as free parameters. Note that an implication of lack of stationarity in mean is that the data in first differences will generally depend on individual effects.

In a short panel, steady state assumptions about initial observations are also critical since estimators that impose them lose consistency if the assumptions fail. Moreover, there are relevant applied situations in which a stable process approximates well the dynamics of data, and yet there are theoretical or empirical grounds to believe that the distribution of initial observations does not coincide with the steady state distribution of the process (cf. Hause, 1980, or Barro and Sala-i-Martin, 1995, and discussion in Arellano, 2003a).

\section{Bias-Corrected Conditional Score Estimation}

\subsection{Normal Likelihood Given Initial Observations and Effects}

Under the normality assumption

$$
\begin{aligned}
y_{i t} \mid y_{i}^{0}, \ldots, y_{i(t-1)}, \eta_{i} \sim \mathcal{N}\left(\alpha_{1} y_{i(t-1)}+\ldots+\alpha_{p} y_{i(t-p)}+\eta_{i}, \sigma_{t}^{2}\right) & (t=1, \ldots, T), \\
& (\text { Assumption } G 1)
\end{aligned}
$$

\footnotetext{
${ }^{3}$ That is, when the roots of the equation $z^{p}-\alpha_{1} z^{p-1}-\ldots-\alpha_{p}=0$ are inside the unit circle.

${ }^{4}$ With the addition of homoskedasticity $\gamma_{00}=\sigma_{\eta}^{2} /(1-\alpha)^{2}+\sigma^{2} /\left(1-\alpha^{2}\right)$.
} 
the $\log$ density of $y_{i}$ conditioned on $\left(y_{i}^{0}, \eta_{i}\right)$ is given by

$$
\ln f\left(y_{i} \mid y_{i}^{0}, \eta_{i}\right)=-\frac{1}{2} \ln \operatorname{det} \Lambda-\frac{1}{2} v_{i}^{\prime} \Lambda^{-1} v_{i}
$$

where $\Lambda$ is a diagonal matrix with elements $\left(\sigma_{1}^{2}, \ldots, \sigma_{T}^{2}\right)$.

The MLE of $\eta_{i}$ for given $\alpha, \sigma_{1}^{2}, \ldots, \sigma_{T}^{2}$ that maximizes (3.1) is

$$
\widehat{\eta}_{i}=\bar{y}_{i}-\bar{x}_{i}^{\prime} \alpha
$$

where $\bar{y}_{i}$ denotes a weighted average $\bar{y}_{i}=\sum_{t=1}^{T} \varphi_{t} y_{i t}$ with weights

$$
\varphi_{t}=\frac{\sigma_{t}^{-2}}{\sigma_{1}^{-2}+\ldots+\sigma_{T}^{-2}}
$$

Concentrating the log likelihood function with respect to the individual effects we obtain

$$
L^{*}=\frac{N}{2} \ln \operatorname{det} \Phi-\frac{N T}{2} \ln \omega_{T}-\frac{1}{2 \omega_{T}} \sum_{i=1}^{N} v_{i}^{\prime}\left(\Phi-\Phi \iota \iota^{\prime} \Phi\right) v_{i}
$$

where $\Phi$ is a diagonal matrix with elements $\left(\varphi_{1}, \ldots, \varphi_{T}\right)$ and $\omega_{T}$ is the variance of the weighted average error:

$$
\omega_{T}=\operatorname{Var}\left(\bar{v}_{i}\right)=\frac{1}{\sigma_{1}^{-2}+\ldots+\sigma_{T}^{-2}} .
$$

It is useful at this point to note that the following identities hold:

$$
\begin{aligned}
v_{i}^{\prime} D^{\prime}\left(D \Lambda D^{\prime}\right)^{-1} D v_{i} & =\frac{1}{\omega_{T}} v_{i}^{\prime}\left(\Phi-\Phi \iota \iota^{\prime} \Phi\right) v_{i}=\sum_{t=1}^{T} \frac{\left(v_{i t}-\bar{v}_{i}\right)^{2}}{\sigma_{t}^{2}} \\
\ln \operatorname{det}\left(D \Lambda D^{\prime}\right) & =-\ln \operatorname{det} \Phi+(T-1) \ln \omega_{T}
\end{aligned}
$$

where $D$ is the $(T-1) \times T$ first-difference matrix operator. Thus, $L^{*}$ can be equally regarded as a function of the data in first differences or in deviations from (weighted) means. Note that with $T=3$ (i.e. $(3+p)$ time series observations per unit), $D \Lambda D^{\prime}$ is unrestricted:

$$
D \Lambda D^{\prime}=\left(\begin{array}{cc}
\sigma_{1}^{2}+\sigma_{2}^{2} & -\sigma_{2}^{2} \\
-\sigma_{2}^{2} & \sigma_{2}^{2}+\sigma_{3}^{2}
\end{array}\right) .
$$


Moreover, the relationship between period-specific and within-group variances is given by

$$
\sigma_{t}^{2}=E\left[\left(v_{i t}-\bar{v}_{i}\right)^{2}\right]+\omega_{T} \quad(t=1, \ldots T) .
$$

The MLE of $\alpha$ for given weights is the following heteroskedastic within-groups estimator

$$
\widehat{\alpha}=\left[\sum_{i=1}^{N} \sum_{t=1}^{T} \varphi_{t}\left(x_{i t}-\bar{x}_{i}\right)\left(x_{i t}-\bar{x}_{i}\right)^{\prime}\right]^{-1} \sum_{i=1}^{N} \sum_{t=1}^{T} \varphi_{t}\left(x_{i t}-\bar{x}_{i}\right)\left(y_{i t}-\bar{y}_{i}\right),
$$

which in first differences can also be written as

$$
\widehat{\alpha}=\left[\sum_{i=1}^{N} X_{i}^{\prime} D^{\prime}\left(D \Lambda D^{\prime}\right)^{-1} D X_{i}\right]^{-1} \sum_{i=1}^{N} X_{i}^{\prime} D^{\prime}\left(D \Lambda D^{\prime}\right)^{-1} D y_{i} .
$$

Finally, the MLE of $\omega_{T}$ for given weights is

$$
\widehat{\omega}_{T}=\frac{1}{T N} \sum_{i=1}^{N} \sum_{t=1}^{T} \varphi_{t}\left(v_{i t}-\bar{v}_{i}\right)^{2} .
$$

Note that, in common with the situation under homoskedasticity, both $\widehat{\alpha}$ and $\widehat{\omega}_{T}$ suffer from the incidental parameters problem. Firstly, although $x_{i t}$ and $v_{i t}$ are orthogonal, their deviations, $\left(x_{i t}-\bar{x}_{i}\right)$ and $\left(v_{i t}-\bar{v}_{i}\right)$, are not, leading to a bias in $\widehat{\alpha}$. Secondly, $\widehat{\omega}_{T}$ evaluated at the true errors and weights will be inconsistent for fixed $T$ due to lack of degrees of freedom adjustment, as evidenced by the equality

$$
\omega_{T}=E\left[\frac{1}{(T-1)} \sum_{t=1}^{T} \varphi_{t}\left(v_{i t}-\bar{v}_{i}\right)^{2}\right] .
$$

\subsection{Likelihood Conditioned on the ML Estimates of the Effects}

Provided G1 holds, the ML estimates of the effects at the true values of the common parameters $\widehat{\eta}_{i}=\eta_{i}+\bar{v}_{i}$ satisfy

$$
\widehat{\eta}_{i} \mid y_{i}^{0}, \eta_{i} \sim \mathcal{N}\left(\eta_{i}, \omega_{T}\right) .
$$

Moreover, the conditional log density of $y_{i}$ given $y_{i 0}, \eta_{i}, \widehat{\eta}_{i}$ is given by

$$
\ln f\left(y_{i} \mid y_{i}^{0}, \eta_{i}, \widehat{\eta}_{i}\right)=-\frac{1}{2} \ln \operatorname{det}\left(D \Lambda D^{\prime}\right)-\frac{1}{2} v_{i}^{\prime} D^{\prime}\left(D \Lambda D^{\prime}\right)^{-1} D v_{i},
$$


which is a within-group density that does not depend on $\eta_{i}$. Thus, (3.1) admits the decomposition

$$
f\left(y_{i} \mid y_{i}^{0}, \eta_{i}\right)=f\left(y_{i} \mid y_{i}^{0}, \widehat{\eta}_{i}\right) f\left(\widehat{\eta}_{i} \mid y_{i}^{0}, \eta_{i}\right),
$$

which confines the dependence on $\eta_{i}$ to the conditional density of $\widehat{\eta}_{i}$. Similarly, any marginal density for $y_{i} \mid y_{i}^{0}$ which imposes a prior distribution on the effects can be written as

$$
f\left(y_{i} \mid y_{i}^{0}\right)=f\left(y_{i} \mid y_{i}^{0}, \widehat{\eta}_{i}\right) f\left(\widehat{\eta}_{i} \mid y_{i}^{0}\right) .
$$

The log likelihood conditioned on $\widehat{\eta}_{i}$ is therefore given by

$$
L_{C}=\frac{N}{2} \ln \operatorname{det} \Phi-\frac{N(T-1)}{2} \ln \omega_{T}-\frac{1}{2 \omega_{T}} \sum_{i=1}^{N} v_{i}^{\prime}\left(\Phi-\Phi \iota \iota^{\prime} \Phi\right) v_{i}
$$

or

$$
L_{C}=-\frac{N}{2} \ln \operatorname{det}\left(D \Lambda D^{\prime}\right)-\frac{1}{2} \sum_{i=1}^{N} v_{i}^{\prime} D^{\prime}\left(D \Lambda D^{\prime}\right)^{-1} D v_{i}
$$

which is similar to the concentrated likelihood (3.4) except that it incorporates a correction for degrees of freedom. In a model with strictly exogenous $x_{i t}, L_{C}$ coincides with the likelihood conditioned on sufficient statistics for the effects, which provides consistent estimates of both the regression and residual variance parameters. However, in the autoregressive situation, the estimator of $\alpha$ that maximizes $L_{C}$ satisfies a heteroskedastic within-group equation of the same form as (3.9) and is therefore inconsistent for fixed $T$.

Inference from a likelihood conditioned on the ML estimates of the effects may lead to consistent estimates provided the scores of the common parameters and the effects are uncorrelated (Cox and Reid, 1987). Cox and Reid's approximate conditional likelihood approach was motivated by the fact that in an exponential family model, it is optimal to condition on sufficient statistics for the nuisance parameters, and these can be regarded as the MLE of nuisance parameters chosen in a form to be orthogonal to the parameters of interest. From this perspective, the inconsistency of within-groups in the autoregressive model results from lack of orthogonality between the scores of $\alpha$ and the effects.

In the homoskedastic case with $p=1$, Lancaster (2002) showed that a likelihood conditioned on the ML estimate of an orthogonalized effect led to a biascorrected score and a consistent method-of-moments estimator under homoskedasticity. Following a similar approach, we construct a heteroskedasticity-consistent 
estimator as the solution to a bias corrected version of the first-order conditions from the likelihood conditioned on the MLE of the effects.

First-Order Conditions The derivatives of $L_{C}$ with respect to $\alpha$ and $\theta=$ $\left(\sigma_{1}^{2} \ldots \sigma_{T}^{2}\right)^{\prime}$ are given by

$$
\begin{gathered}
\frac{\partial L_{C}}{\partial \alpha}=\sum_{i=1}^{N} X_{i}^{\prime} D^{\prime}\left(D \Lambda D^{\prime}\right)^{-1} D v_{i} \\
\frac{\partial L_{C}}{\partial \theta}=\frac{1}{2} \sum_{i=1}^{N} K^{\prime}\left(D \Lambda D^{\prime} \otimes D \Lambda D^{\prime}\right)^{-1} \operatorname{vec}\left(D v_{i} v_{i}^{\prime} D^{\prime}-D \Lambda D^{\prime}\right)
\end{gathered}
$$

where $K$ is a $(T-1)^{2} \times T$ selection matrix such that vec $\left(D \Lambda D^{\prime}\right)=K \theta$. Let $d_{t}$ and $k_{t}$ be the $t$-th columns of $D$ and $K$, respectively, so that $D \Lambda D^{\prime}=\sum_{t=1}^{T} \sigma_{t}^{2} d_{t} d_{t}^{\prime}$, $K \theta=\sum_{t=1}^{T} \sigma_{t}^{2} k_{t}$, and $k_{t}=d_{t} \otimes d_{t}$. Thus, also

$$
\frac{\partial L_{C}}{\partial \sigma_{t}^{2}}=\frac{1}{2} \sum_{i=1}^{N} d_{t}^{\prime}\left(D \Lambda D^{\prime}\right)^{-1}\left(D v_{i} v_{i}^{\prime} D^{\prime}-D \Lambda D^{\prime}\right)\left(D \Lambda D^{\prime}\right)^{-1} d_{t} \quad(t=1, \ldots, T) .
$$

Maximizing $L_{C}$ with respect to $\omega_{T}$ and $\left(\varphi_{1} \ldots \varphi_{T}\right)$ for given $\alpha$, subject to the adding-up restriction $\iota^{\prime} \Phi \iota=1$, the first-order conditions for variance parameters can also be written in a form analogous to (3.8) and (3.11) as

$$
\begin{aligned}
\sum_{i=1}^{N}\left[\frac{1}{(T-1)} v_{i}^{\prime}\left(\Phi-\Phi \iota \iota^{\prime} \Phi\right) v_{i}-\omega_{T}\right] & =0 \\
\sum_{i=1}^{N}\left[\left(v_{i t}-\bar{v}_{i}\right)^{2}-\left(v_{i(t-1)}-\bar{v}_{i}\right)^{2}-\left(\sigma_{t}^{2}-\sigma_{t-1}^{2}\right)\right] & =0 \quad(t=2, \ldots, T) .
\end{aligned}
$$

Thus, the conditional MLE of $\alpha$ and $\theta$ solve, respectively, (3.10) and

$$
\widehat{\theta}=\left(K^{\prime} \Upsilon^{-1} K\right)^{-1} K^{\prime} \Upsilon^{-1} \frac{1}{N} \sum_{i=1}^{N} \operatorname{vec}\left(D v_{i} v_{i}^{\prime} D^{\prime}\right)
$$

where $\Upsilon=D \Lambda D^{\prime} \otimes D \Lambda D^{\prime}$

Bias corrected conditional ML scores Under Assumption $A$ the expected conditional ML scores are given by

$$
E\left[X_{i}^{\prime} D^{\prime}\left(D \Lambda D^{\prime}\right)^{-1} D v_{i}\right]=-h_{T}(\alpha, \varphi)
$$




$$
E\left[K^{\prime}\left(D \Lambda D^{\prime} \otimes D \Lambda D^{\prime}\right)^{-1} \operatorname{vec}\left(D v_{i} v_{i}^{\prime} D^{\prime}-D \Lambda D^{\prime}\right)\right]=0
$$

where

$$
h_{T}(\alpha, \varphi)=\left(\begin{array}{c}
\varphi^{\prime} C_{1} \iota \\
\vdots \\
\varphi^{\prime} C_{p} \iota
\end{array}\right)
$$

with

$$
C_{j}=\left(\begin{array}{cc}
0 & 0 \\
B_{T-j}^{-1} & 0
\end{array}\right)
$$

and $B_{T-j}$ is a $(T-j) \times(T-j)$ matrix such that

$$
B_{T-j}=\left(\begin{array}{ccccccc}
1 & 0 & \ldots & 0 & \ldots & 0 & 0 \\
-\alpha_{1} & 1 & & 0 & \ldots & 0 & 0 \\
-\alpha_{2} & -\alpha_{1} & \ddots & 0 & \ldots & 0 & 0 \\
& \ddots & \ddots & \ddots & & \vdots & \vdots \\
0 & & & 0 & & 1 & 0 \\
0 & 0 & \ldots & -\alpha_{p} & \ldots & -\alpha_{1} & 1
\end{array}\right) .
$$

When $p=1, h_{T}(\alpha, \varphi)$ is a scalar function given by

$$
h_{T}(\alpha, \varphi)=\sum_{t=1}^{T-1}\left(1+\alpha+\ldots+\alpha^{t-1}\right) \varphi_{t+1} .
$$

Under homoskedasticity $\varphi_{t}=T^{-1}$ for all $t$, and the bias function (3.29) boils down to the expression in Nickell (1981) and Lancaster (2002), which for $|\alpha|<1$ is $^{5}$

$$
h_{T}(\alpha)=\frac{1}{(1-\alpha)}\left[1-\frac{1}{T}\left(\frac{1-\alpha^{T}}{1-\alpha}\right)\right] .
$$

In view of (3.24)-(3.25), heteroskedasticity-consistent GMM estimators can be obtained as the solution to the nonlinear estimating equations

$$
\begin{aligned}
\sum_{i=1}^{N} X_{i}^{\prime} D^{\prime}\left(D \Lambda D^{\prime}\right)^{-1} D v_{i}+N h_{T}(\alpha, \varphi) & =0 \\
K^{\prime}\left(D \Lambda D^{\prime} \otimes D \Lambda D^{\prime}\right)^{-1} v e c \sum_{i=1}^{N}\left(D v_{i} v_{i}^{\prime} D^{\prime}-D \Lambda D^{\prime}\right) & =0 .
\end{aligned}
$$

\footnotetext{
${ }^{5}$ Note that although the bias of the CML scores only depends on $(\alpha, \varphi)$, the asymptotic bias of the CML estimator of $\alpha$ as $N \rightarrow \infty$ also depends on the covariance matrix of $\left(\eta_{i}, y_{i}^{0}\right)$. Approximate bias formulae for homoskedastic WG were derived by Hahn and Kuersteiner (2002), and Alvarez and Arellano (2003). A bias-corrected estimator so constructed removes bias to order $T^{-2}$ but is not fixed- $T$ consistent.
} 
Consistency of the bias-corrected score estimator (BCS) that solves (3.31)-(3.32) does not depend on normality nor on conditional or time-series homoskedasticity.

BCS estimation is not possible from a $(2+p)$-wave panel (i.e. $T=2)$ because in that case $\alpha$ is not identified from the expected scores, which for $p=1$ are given by

$$
\begin{aligned}
E\left[\left(y_{i 1}-y_{i 0}\right)\left(v_{i 2}-v_{i 1}\right)\right] & =-\sigma_{1}^{2} \\
E\left[\left(v_{i 2}-v_{i 1}\right)^{2}\right] & =\sigma_{1}^{2}+\sigma_{2}^{2} .
\end{aligned}
$$

This situation is in contrast with Lancaster's BCS estimator that enforces time series homoskedasticity (hence achieving identification from (3.33)-(3.34)), or the bias-corrected within-group estimator considered in Kiviet (1995).

\subsection{Modified Conditional Likelihood Interpretation}

If the weights $\varphi$ are known and $p=1$, the method of moments estimators of $\alpha$ and $\omega_{T}$ based on the bias corrected scores

$$
\begin{aligned}
& E\left[x_{i}^{\prime} D^{\prime}\left(D \Phi^{-1} D^{\prime}\right)^{-1} D v_{i}\right]=-\omega_{T} h_{T}(\alpha, \varphi) \\
& E\left[v_{i}^{\prime} D^{\prime}\left(D \Phi^{-1} D^{\prime}\right)^{-1} D v_{i}\right]=(T-1) \omega_{T}
\end{aligned}
$$

can be regarded as the maximizers of the criterion function

$$
L_{C R}=L_{C}+N b_{T}(\alpha, \varphi)
$$

where

$$
b_{T}(\alpha, \varphi)=\sum_{t=1}^{T-1} \frac{\left(\varphi_{t+1}+\ldots+\varphi_{T}\right)}{t} \alpha^{t},
$$

which is the integral of $h_{T}(\alpha, \varphi)$ up to an arbitrary constant of integration that may depend on $\varphi$.

Following Lancaster (2002), $L_{C R}$ can be interpreted as a Cox-Reid likelihood conditioned on the ML estimate $\widehat{\lambda}_{i}$ of an orthogonal effect $\lambda_{i}$ (Arellano, 2003a, p. 105)

$$
L_{C R}=\sum_{i=1}^{N} \ln f\left(y_{i} \mid y_{i 0}, \widehat{\lambda}_{i}\right)
$$


or as an integrated likelihood

$$
L_{C R}=\sum_{i=1}^{N} \ln f\left(y_{i} \mid y_{i 0}\right)=\sum_{i=1}^{N} \ln f\left(y_{i} \mid y_{i 0}, \widehat{\eta}_{i}\right)+\sum_{i=1}^{N} \ln f\left(\widehat{\eta}_{i} \mid y_{i 0}\right)
$$

in which the chosen prior distribution of the effects conditioned on $y_{i 0}$ is such that the marginal density of $\widehat{\eta}_{i} \mid y_{i 0}$ satisfies:

$$
f\left(\widehat{\eta}_{i} \mid y_{i 0}\right)=\kappa_{i}(\varphi) e^{b_{T}(\alpha, \varphi)}
$$

where $\kappa_{i}(\varphi)$ is a version of the constant of integration.

The first interpretation is based on a decomposition conditional on $\widehat{\lambda}_{i}$ similar to (3.14), whereas the second relies on factorization (3.15).

With unknown weights and $p>1$ there is no orthogonal reparameterization, but we show in Appendix $\mathrm{C}$ that for a heteroskedastic $\operatorname{AR}(p)$ model with unknown weights, the BCS estimating equations coincide with the modified score vector discussed in Arellano (2003b). Thus, in our setting a first-order bias adjustment to the score is an exact correction that removes fully the bias, hence leading to fixed- $T$ consistency.

\section{Random Effects Estimation}

The analysis so far was conditional on $y_{i}^{0}$ and $\widehat{\eta}_{i}$. Conditioning on $y_{i}^{0}$ avoided steady state restrictions, but by conditioning on $\widehat{\eta}_{i}$ estimation is exclusively based on the data in first-differences. We now turn to explore marginal maximum likelihood estimation based on a normal prior distribution of the effects conditioned on $y_{i}^{0}$, with linear mean and constant variance. A sufficient condition that we use for simplicity is:

Assumption $G 2:\left(\eta_{i}, y_{i}^{0}\right)$ is jointly normally distributed with an unrestricted covariance matrix.

Normality of $y_{i}^{0}$ is unessential because its variance matrix is a free parameter, so the following analysis can be regarded as conditional on $y_{i}^{0}$. Clearly, assumptions $G 1$ and $G 2$ together imply that $\left(\eta_{i}, y_{i}^{0}, y_{i 1}, \ldots, y_{i T}\right)$ are jointly normally distributed.

The random effects log likelihood Under $G 2$,

$$
\widehat{\eta}_{i} \mid y_{i}^{0} \sim \mathcal{N}\left(\phi^{\prime} y_{i}^{0}, \sigma_{\varepsilon}^{2}\right)
$$


where $\phi=\Gamma_{00}^{-1} \gamma_{\eta 0}$ and $\sigma_{\varepsilon}^{2}=\omega_{T}+\sigma_{\eta}^{2}-\gamma_{\eta 0}^{\prime} \Gamma_{00}^{-1} \gamma_{\eta 0}$. So, using factorization (3.15), the density of $y_{i}$ conditioned on $y_{i}^{0}$ but marginal on $\eta_{i}$ is:

$$
\begin{aligned}
\ln f\left(y_{i} \mid y_{i}^{0}\right)= & -\frac{1}{2} \ln \operatorname{det}\left(D \Lambda D^{\prime}\right)-\frac{1}{2} v_{i}^{\prime} D^{\prime}\left(D \Lambda D^{\prime}\right)^{-1} D v_{i} \\
& -\frac{1}{2} \ln \sigma_{\varepsilon}^{2}-\frac{1}{2 \sigma_{\varepsilon}^{2}}\left(\bar{y}_{i}-\alpha^{\prime} \bar{x}_{i}-\phi^{\prime} y_{i}^{0}\right)^{2} .
\end{aligned}
$$

Thus, letting $\bar{u}_{i}=\bar{y}_{i}-\alpha^{\prime} \bar{x}_{i}$, the random effects log likelihood is a function of $\left(\alpha, \sigma_{1}^{2}, \ldots, \sigma_{T}^{2}, \phi, \sigma_{\varepsilon}^{2}\right)$ given by

$$
L_{R}=L_{C}-\frac{N}{2} \ln \sigma_{\varepsilon}^{2}-\frac{1}{2 \sigma_{\varepsilon}^{2}} \sum_{i=1}^{N}\left(\bar{u}_{i}-\phi^{\prime} y_{i}^{0}\right)^{2},
$$

with scores:

$$
\begin{aligned}
\frac{\partial L_{R}}{\partial \alpha} & =\frac{\partial L_{C}}{\partial \alpha}+\frac{1}{\sigma_{\varepsilon}^{2}} \sum_{i=1}^{N} \bar{x}_{i}\left(\bar{u}_{i}-\phi^{\prime} y_{i}^{0}\right) \\
\frac{\partial L_{R}}{\partial \theta} & =\frac{\partial L_{C}}{\partial \theta}+\frac{1}{\sigma_{\varepsilon}^{2}} \sum_{i=1}^{N} \Phi D^{\prime}\left(D \Lambda D^{\prime}\right)^{-1} D u_{i}\left(\bar{u}_{i}-\phi^{\prime} y_{i}^{0}\right) \\
\frac{\partial L_{R}}{\partial \phi} & =\frac{1}{\sigma_{\varepsilon}^{2}} \sum_{i=1}^{N} y_{i}^{0}\left(\bar{u}_{i}-\phi^{\prime} y_{i}^{0}\right) \\
\frac{\partial L_{R}}{\partial \sigma_{\varepsilon}^{2}} & =\frac{1}{2 \sigma_{\varepsilon}^{4}} \sum_{i=1}^{N}\left[\left(\bar{u}_{i}-\phi^{\prime} y_{i}^{0}\right)^{2}-\sigma_{\varepsilon}^{2}\right]
\end{aligned}
$$

Under Assumption $A$ the expectations of the second terms in the scores for $\alpha$ and $\theta$ at true values are:

$$
E\left[\frac{1}{\sigma_{\varepsilon}^{2}} \bar{x}_{i}\left(\bar{u}_{i}-\phi^{\prime} y_{i}^{0}\right)\right]=h_{T}(\alpha, \varphi)
$$

and

$$
E\left[\frac{1}{\sigma_{\varepsilon}^{2}} \Phi D^{\prime}\left(D \Lambda D^{\prime}\right)^{-1} D v_{i}\left(\bar{u}_{i}-\phi^{\prime} y_{i}^{0}\right)\right]=0 .
$$

Therefore, in view of (3.24) and (3.25), under Assumption $A$ the expected scores evaluated at the true values of the parameters are equal to zero:

$$
E\left[X_{i}^{\prime} D^{\prime}\left(D \Lambda D^{\prime}\right)^{-1} D v_{i}+\frac{1}{\sigma_{\varepsilon}^{2}} \bar{x}_{i}\left(\bar{u}_{i}-\phi^{\prime} y_{i}^{0}\right)\right]=0
$$




$$
\begin{gathered}
E\left[\frac{1}{2} K^{\prime}\left(D \Lambda D^{\prime} \otimes D \Lambda D^{\prime}\right)^{-1} \operatorname{vec}\left(D v_{i} v_{i}^{\prime} D^{\prime}-D \Lambda D^{\prime}\right)\right. \\
\left.+\frac{1}{\sigma_{\varepsilon}^{2}} \Phi D^{\prime}\left(D \Lambda D^{\prime}\right)^{-1} D v_{i}\left(\bar{u}_{i}-\phi^{\prime} y_{i}^{0}\right)\right]=0 \\
E\left[y_{i}^{0}\left(\bar{u}_{i}-\phi^{\prime} y_{i}^{0}\right)\right]=0 \\
E\left[\left(\bar{u}_{i}-\phi^{\prime} y_{i}^{0}\right)^{2}-\sigma_{\varepsilon}^{2}\right]=0
\end{gathered}
$$

The random effects maximum likelihood estimator (RML) solves the estimating equations (4.4)-(4.7) and is consistent and asymptotically normal under assumption $A$ regardless of non-normality or conditional heteroskedasticity.

In a $(2+p)$-wave panel $(T=2)$ the model is just-identified and the RML estimator coincides with the Anderson-Hsiao (1981) estimator based on the instrumentalvariable conditions

$$
E\left[y_{i}^{0}\left(\Delta y_{i 2}-\alpha_{1} \Delta y_{i 1}-\ldots-\alpha_{p} \Delta y_{i(2-p)}\right)\right]=0 .
$$

Random effects likelihood functions for homoskedastic autoregressive models under the normality assumption $G 2$ have been considered in Chamberlain (1980, 234-235), Blundell and Smith (1991), Sims (2000), and Alvarez and Arellano (2003).

Efficiency Comparisons In order to compare the relative efficiency of the BCS and RML estimators, it is useful to notice that RML is asymptotically equivalent to an overidentified GMM estimator that uses the moment conditions:

$$
\begin{gathered}
E\left[X_{i}^{\prime} D^{\prime}\left(D \Lambda D^{\prime}\right)^{-1} D v_{i}\right]=-h_{T}(\alpha, \varphi) \\
E\left[K^{\prime}\left(D \Lambda D^{\prime} \otimes D \Lambda D^{\prime}\right)^{-1} \operatorname{vec}\left(D v_{i} v_{i}^{\prime} D^{\prime}-D \Lambda D^{\prime}\right)\right]=0 \\
E\left[\frac{1}{\sigma_{\varepsilon}^{2}} \bar{x}_{i}\left(\bar{u}_{i}-\phi^{\prime} y_{i}^{0}\right)\right]=h_{T}(\alpha, \varphi) \\
E\left[D^{\prime}\left(D \Lambda D^{\prime}\right)^{-1} D v_{i}\left(\bar{u}_{i}-\phi^{\prime} y_{i}^{0}\right)\right]=0 \\
E\left[y_{i}^{0}\left(\bar{u}_{i}-\phi^{\prime} y_{i}^{0}\right)\right]=0 \\
E\left[\left(\bar{u}_{i}-\phi^{\prime} y_{i}^{0}\right)^{2}-\sigma_{\varepsilon}^{2}\right]=0 .
\end{gathered}
$$


and a weight matrix calculated under the assumption of normality. ${ }^{6}$

BCS is based on moments (4.11) and (4.12), but RML is also using the information from the data in levels contained in (4.13) and (4.14). Moments (4.13) give the between-group covariance between the regressors and the error, in the same way as the BCS moments (4.11) specified the within-group covariance. The moments in (4.14) state the orthogonality between within-group and betweengroup errors (partialling out the initial observations). Finally, (4.15) and (4.16) are unrestricted moments that determine $\phi$ and $\sigma_{\varepsilon}^{2}$.

Therefore, if the data are normally distributed RML is asymptotically more efficient than BCS. Otherwise, they cannot be ordered. Nevertheless, a GMM estimator based on (4.11)-(4.16) and a robust weight matrix that remains optimal under nonnormality will never be less efficient asymptotically than BCS, and may achieve a significant reduction in the number of moments relative to standard GMM procedures.

The concentrated random effects log-likelihood Concentrating $L_{R}$ with respect to $\sigma_{\varepsilon}^{2}$ and $\phi$ we obtain the following criterion function that only depends on $\alpha$ and $\theta$ :

$$
L_{R}^{*}=L_{C}-\frac{N}{2} \ln \left[\left(\bar{y}-\alpha^{\prime} \bar{x}\right)^{\prime} S_{0}\left(\bar{y}-\alpha^{\prime} \bar{x}\right)\right]
$$

where $S_{0}=I_{N}-Y_{0}\left(Y_{0}^{\prime} Y_{0}\right)^{-1} Y_{0}^{\prime}$, and $Y_{0}=\left(y_{1}^{0}, \ldots, y_{N}^{0}\right)^{\prime}$.

$L_{R}^{*}$ can be regarded as a modified heteroskedastic within-group criterion with a correction term that becomes less important as $T$ increases.

A linear (OLS) Estimator of Variance Weights A simple consistent estimator of the variance weights for given $\alpha$ can be obtained from the fact that $E\left(\bar{u}_{i} \Delta v_{i t}\right)=0$. Such estimator may be useful for providing starting values for nonlinear likelihood-based estimation.

Enforcing the adding-up constraint, the average error can be written as

$$
\begin{aligned}
\bar{u}_{i} & =\varphi_{1} u_{i 1}+\ldots+\varphi_{T-1} u_{i(T-1)}+\left(1-\varphi_{1}-\ldots-\varphi_{T-1}\right) u_{i T} \\
& =u_{i T}-\varphi_{1}\left(u_{i T}-u_{i 1}\right)-\ldots-\varphi_{T-1}\left(u_{i T}-u_{i(T-1)}\right) .
\end{aligned}
$$

\footnotetext{
${ }^{6}$ Interestingly, $\left(\sigma_{1}^{2}, \sigma_{2}^{2}\right)$ are identified from the RML scores when $T=2$. In that case (4.12) determines $\left(\sigma_{1}^{2}+\sigma_{2}^{2}\right)$ and (4.14) determines $\varphi_{1}$. Note that when $T=2$ one of the two moments in (4.12) is redundant.
} 
Letting $\varphi_{o}=\left(\varphi_{1}, \ldots, \varphi_{T-1}\right)^{\prime}$ and $w_{i}=\left[\left(u_{i T}-u_{i 1}\right), \ldots,\left(u_{i T}-u_{i(T-1)}\right)\right]^{\prime}$, we have orthogonality between $w_{i}$ and $\bar{u}_{i}$

$$
E\left[w_{i}\left(u_{i T}-w_{i}^{\prime} \varphi_{o}\right)\right]=0
$$

which suggests the following OLS estimator of $\varphi_{o}$ for given $\alpha$ :

$$
\widetilde{\varphi}_{o}=\left(\sum_{i=1}^{N} w_{i} w_{i}^{\prime}\right)^{-1} \sum_{i=1}^{N} w_{i} u_{i T} .
$$

This estimator satisfies the adding-up constraint, but not necessarily the nonnegativity restrictions.

Given the $\widetilde{\varphi}_{t}$ 's, estimates of $\omega_{T}$ and the $\sigma_{t}^{2}$ 's can be obtained from

$$
\begin{aligned}
\widetilde{\omega}_{T} & =\frac{1}{(T-1) N} \sum_{i=1}^{N} \sum_{t=1}^{T} \widetilde{\varphi}_{t}\left(v_{i t}-\bar{v}_{i}\right)^{2} \\
\widetilde{\sigma}_{t}^{2} & =\frac{\widetilde{\omega}_{T}}{\widetilde{\varphi}_{t}} .
\end{aligned}
$$

\section{Estimation from the Data in Differences}

Until now, the starting point was an interest in the conditional distribution of $\left(y_{i 1}, \ldots, y_{i T}\right)$ given $y_{i}^{0}$ and $\eta_{i}$ under the assumption that $y_{i}^{0}$ was observed but $\eta_{i}$ was not. That is, that the data consisted on a random sample of the vectors $\left(y_{i}^{0 \prime}, y_{i 1}, \ldots, y_{i T}\right)$. In this section we maintain the interest in the same conditional distribution as before, but assume that only changes of the $y_{i t}$ variables are observed, so that the data on individual $i$ is $\left(\Delta y_{i(2-p)}, \ldots, \Delta y_{i T}\right)$. This situation is clearly relevant when the data source only provides information on changes, but it may also be interesting if it is thought that an analysis based on changes is more "robust" than one based on levels. An objective of this and the next section is to discuss the content of this intuition by relating ML in differences to the previous conditional and marginal methods. Maximum likelihood estimation of autoregressive models using first-differences has been considered by Hsiao, Pesaran, and Tahmiscioglu (2002).

As a matter of notation, note that observability of $\left(\Delta y_{i(2-p)}, \ldots, \Delta y_{i T}\right)$ is equivalent to observing $\left(y_{i(2-p)}^{\dagger}, \ldots, y_{i T}^{\dagger}\right)^{\prime}=\left(y_{i(2-p)}-y_{i(1-p)}, \ldots, y_{i T}-y_{i(1-p)}\right)^{\prime}$, since the 
former results from multiplying the latter by the nonsingular transformation matrix of order $(T+p-1)$ :

$$
D^{\dagger}=\left(\begin{array}{ccccc}
1 & 0 & \cdots & 0 & 0 \\
& & D & &
\end{array}\right)
$$

with $\operatorname{det}\left(D^{\dagger}\right)=1$. Also note that by construction $y_{i(1-p)}^{\dagger}=0$.

We shall use the notation $y_{i}^{\dagger}=y_{i}-y_{i(1-p)} \iota_{T}$ and $X_{i}^{\dagger}=X_{i}-y_{i(1-p)} \iota_{T} \iota_{p}^{\prime}$. Similarly, $\bar{y}_{i}^{\dagger}=y_{i}^{\dagger} \Phi_{\iota_{T}}=\bar{y}_{i}-y_{i(1-p)}$, etc. The following is an expression of $\bar{y}_{i}^{\dagger}$ that makes explicit the connection to the data in differences:

$$
\bar{y}_{i}^{\dagger}=\sum_{j=1}^{p} \Delta y_{i(1-p)+j}+\sum_{t=2}^{T}\left(\sum_{s=t}^{T} \varphi_{s}\right) \Delta y_{i t} .
$$

The original model can be written as

$$
\begin{aligned}
y_{i 1}^{\dagger} & =\alpha_{1} y_{i 0}^{\dagger}+\ldots+\alpha_{p-1} y_{i(2-p)}^{\dagger}+\eta_{i}^{\dagger}+v_{i 1} \\
y_{i t}^{\dagger} & =\alpha_{1} y_{i(t-1)}^{\dagger}+\ldots+\alpha_{p} y_{i(t-p)}^{\dagger}+\eta_{i}^{\dagger}+v_{i t} \quad(t=2, \ldots, T) .
\end{aligned}
$$

where

$$
\eta_{i}^{\dagger}=\eta_{i}-\left(1-\alpha_{1}-\ldots-\alpha_{p}\right) y_{i(1-p)} .
$$

Thus, the model for the deviations $y_{i t}^{\dagger}$ can be regarded as a version of the original model in which $y_{i(1-p)}^{\dagger}=0$ for all individuals and the effect is given by $\eta_{i}^{\dagger}$. From the point of view of this section, bundling together $y_{i(1-p)}$ and $\eta_{i}$ into $\eta_{i}^{\dagger}$ makes sense because they are both unobserved. The usefulness of this notation is that it allows us to easily obtain densities for the variables in first differences relying on the previous results for the levels.

Since the shocks $v_{i t}$ remain the same in representation (5.2)-(5.3), applying (3.13) we have

$$
\ln f\left(y_{i}^{\dagger} \mid y_{i}^{0 \dagger}, \eta_{i}^{\dagger}, \widehat{\eta}_{i}^{\dagger}\right)=-\frac{1}{2} \ln \operatorname{det}\left(D \Lambda D^{\prime}\right)-\frac{1}{2} v_{i}^{\prime} D^{\prime}\left(D \Lambda D^{\prime}\right)^{-1} D v_{i}
$$

where at true values

$$
\widehat{\eta}_{i}^{\dagger}=\bar{y}_{i}^{\dagger}-\alpha^{\prime} \bar{x}_{i}^{\dagger}=\eta_{i}^{\dagger}+\bar{v}_{i}=\bar{u}_{i}-\left(1-\alpha^{\prime} \iota_{p}\right) y_{i(1-p)},
$$

and following (3.12):

$$
\widehat{\eta}_{i}^{\dagger} \mid y_{i}^{0 \dagger}, \eta_{i}^{\dagger} \sim \mathcal{N}\left(\eta_{i}^{\dagger}, \omega_{T}\right)
$$


Also, mimicking the marginal density decomposition in (3.15):

$$
f\left(y_{i}^{\dagger} \mid y_{i}^{0 \dagger}\right)=f\left(y_{i}^{\dagger} \mid y_{i}^{0 \dagger}, \widehat{\eta}_{i}^{\dagger}\right) \int f\left(\widehat{\eta}_{i}^{\dagger} \mid y_{i}^{0 \dagger}, \eta_{i}^{\dagger}\right) d G\left(\eta_{i}^{\dagger} \mid y_{i}^{0 \dagger}\right) .
$$

Moreover, since $y_{i(1-p)}^{\dagger}=0$ with probability one, for $p=1$ densities conditioned on $y_{i}^{0 \dagger}$ coincide with unconditional densities, and for $p>1$ conditioning on $y_{i}^{0 \dagger}$ is equivalent to conditioning on $\Delta y_{i}^{0}=\left(\Delta y_{i(2-p)}, \ldots, \Delta y_{i 0}\right)^{\prime}$. Thus, for $p>1$, $f\left(y_{i}^{\dagger} \mid \Delta y_{i}^{0}\right)=f\left(y_{i}^{\dagger} \mid y_{i}^{0 \dagger}\right)$ and

$$
\widehat{\eta}_{i}^{\dagger} \mid \Delta y_{i}^{0}, \eta_{i}^{\dagger} \sim \mathcal{N}\left(\eta_{i}^{\dagger}, \omega_{T}\right),
$$

so that

$$
f\left(y_{i}^{\dagger} \mid \Delta y_{i}^{0}\right)=f\left(y_{i}^{\dagger} \mid \Delta y_{i}^{0}, \widehat{\eta}_{i}^{\dagger}\right) f\left(\widehat{\eta}_{i}^{\dagger} \mid \Delta y_{i}^{0}\right) .
$$

Recall that the density $f\left(y_{i}^{\dagger} \mid \Delta y_{i}^{0}\right)$ is also the density of the first-differences of the data $\left(\Delta y_{i 1}, \ldots, \Delta y_{i T}\right)$ conditioned on $\Delta y_{i}^{0}$, which we are expressing as the product of the usual within-group conditional density and the density of $\widehat{\eta}_{i}^{\dagger}$ conditioned on $\Delta y_{i}^{0}$. Therefore, in the absence of steady state assumptions about initial conditions, the form of the density of panel $\operatorname{AR}(p)$ data in first differences depends on the distribution of the effects. In the next section we shall see that this dependence vanishes under the assumption of mean stationarity.

Let $\left(\phi^{\dagger}, \sigma_{\varepsilon}^{2 \dagger}\right)$ denote the linear regression coefficients of $\widehat{\eta}_{i}^{\dagger}$ on $\Delta y_{i}^{0}$, so that $\sigma_{\varepsilon}^{2 \dagger}$ satisfies

$$
\sigma_{\varepsilon}^{2 \dagger}=\sigma_{\eta^{\dagger}}^{2}+\omega_{T}-\phi^{\dagger \prime} \operatorname{Var}\left(\Delta y_{i}^{0}\right) \phi^{\dagger}
$$

Under the normality assumption $G 2$

$$
\widehat{\eta}_{i}^{\dagger} \mid \Delta y_{i}^{0} \sim \mathcal{N}\left(\phi^{\dagger \prime} \Delta y_{i}^{0}, \sigma_{\varepsilon}^{2 \dagger}\right),
$$

we have the following "random effects" log density for the data in first differences

$$
\begin{aligned}
\ln f\left(\Delta y_{i 1}, \ldots, \Delta y_{i T} \mid \Delta y_{i}^{0}\right)= & -\frac{1}{2} \ln \operatorname{det}\left(D \Lambda D^{\prime}\right)-\frac{1}{2} v_{i}^{\prime} D^{\prime}\left(D \Lambda D^{\prime}\right)^{-1} D v_{i} \\
& -\frac{1}{2} \ln \sigma_{\varepsilon}^{2 \dagger}-\frac{1}{2 \sigma_{\varepsilon}^{2 \dagger}}\left(\bar{y}_{i}^{\dagger}-\bar{x}_{i}^{\dagger} \alpha-\phi^{\dagger \prime} \Delta y_{i}^{0}\right)^{2}(5
\end{aligned}
$$

Therefore, the random effects log likelihood for the data in first-differences is a function of $\left(\alpha, \sigma_{1}^{2}, \ldots, \sigma_{T}^{2}, \sigma_{\varepsilon}^{2 \dagger}, \phi^{\dagger}\right)$ given by

$$
L_{R D}=L_{C}-\frac{N}{2} \ln \sigma_{\varepsilon}^{2 \dagger}-\frac{1}{2 \sigma_{\varepsilon}^{2 \dagger}} \sum_{i=1}^{N}\left(\bar{y}_{i}^{\dagger}-\alpha^{\prime} \bar{x}_{i}^{\dagger}-\phi^{\dagger \prime} \Delta y_{i}^{0}\right)^{2} .
$$


Concentrating $L_{R D}$ with respect to $\sigma_{\varepsilon}^{2 \dagger}$ and $\phi^{\dagger}$, and letting $S_{\Delta}^{0}=I_{N}-Y_{\Delta}^{0}\left(Y_{\Delta}^{0 \prime} Y_{\Delta}^{0}\right)^{-1} Y_{\Delta}^{0 \prime}$ with $Y_{\Delta}^{0}=\left(\Delta y_{1}^{0}, \ldots, \Delta y_{N}^{0}\right)^{\prime}$, we obtain the following criterion function that only depends on $\alpha$ and $\theta$ :

$$
L_{R D}^{*}=L_{C}-\frac{N}{2} \ln \left(\bar{y}^{\dagger}-\alpha^{\prime} \bar{x}^{\dagger}\right)^{\prime} S_{\Delta}^{0}\left(\bar{y}^{\dagger}-\alpha^{\prime} \bar{x}^{\dagger}\right),
$$

which, in common with (4.17), can be regarded as a modified heteroskedastic within-group criterion with a small $T$ correction term.

The random effects ML estimator in first-differences (RML-dif) maximizes $L_{R D}^{*}$ and is consistent and asymptotically normal under assumption $A$ regardless of nonnormality or conditional heteroskedasticity.

In the $p=1$ case, the term $\Delta y_{i}^{0}$ does not occur, so that (5.12) becomes a marginal density for the data in first differences and the log likelihood is just a function of $\left(\alpha, \sigma_{1}^{2}, \ldots, \sigma_{T}^{2}, \sigma_{\varepsilon}^{2 \dagger}\right)$ given by

$$
L_{R D}=L_{C}-\frac{N}{2} \ln \sigma_{\varepsilon}^{2 \dagger}-\frac{1}{2 \sigma_{\varepsilon}^{2 \dagger}} \sum_{i=1}^{N}\left(\bar{y}_{i}^{\dagger}-\alpha \bar{x}_{i}^{\dagger}\right)^{2} .
$$

Underidentification in a $(2+p)$-Wave Panel $(T=2) \quad$ In common with BCS, RML-dif estimation is not possible from a $(2+p)$-wave panel because $\alpha$ is not identified from the expected scores of $L_{R D}$. In contrast, RML achieves identification by relying on the data in levels. The relationship between the two procedures is best illustrated by examining for $p=1$ the covariance matrix of the transformed series

$$
\operatorname{Var}\left(\begin{array}{c}
y_{i 0} \\
\Delta y_{i 1} \\
\Delta y_{i 2}
\end{array}\right)=\Omega^{*}=\left(\begin{array}{lll}
\gamma_{00} & \gamma_{0 \Delta 1} & \gamma_{0 \Delta 2} \\
\gamma_{0 \Delta 1} & & \\
\gamma_{0 \Delta 2} & & \Omega_{\Delta}
\end{array}\right)
$$

where $\Omega^{*}$ is a non-singular transformation of the covariance matrix in levels and $\Omega_{\Delta}$ is the covariance matrix in first-differences. Thus, a model of $\Omega_{\Delta}$ is equivalent to a model of $\Omega^{*}$ that leaves the coefficients $\gamma_{00}, \gamma_{0 \Delta 1}$ and $\gamma_{0 \Delta 2}$ unrestricted (Arellano, 2003a, p. 67). With $T=2$, the only identifying information about $\alpha$ is precisely the restriction $\gamma_{0 \Delta 2}=\alpha \gamma_{0 \Delta 1}$ satisfied by those coefficients, hence lack of identification from $\Omega_{\Delta}$. Under time series homoskedasticity, $\alpha$ is identifiable from $\Omega_{\Delta}$ when $T=2$, but in that case all the information comes from the homoskedasticity assumption. 
Efficiency Comparisons If the data are normally distributed RML is asymptotically more efficient than RML-dif, which in turn is more efficient than BCS. The relative efficiency of RML-dif with respect to BCS under normality is a consequence of the fact that both are statistics of the first differenced data, but the former is the maximum likelihood estimator.

In the absence of normality, the estimators cannot be ranked. However, regardless of normality, under Assumption $A$ estimates based on first-differences alone will never be more efficient than an optimal GMM estimator based on the full covariance structure for the data in levels.

\section{Estimation Under Stationarity in Mean}

In this section we consider conditional and marginal maximum likelihood estimators that allow for time series heteroskedasticity but exploit the stationarity in mean condition discussed in Section 2. Namely, that for every $t$ the mean of $y_{i t}$ conditioned on $\eta_{i}$ coincides with the steady state mean of the process $\mu_{i}=\eta_{i} /\left(1-\alpha^{\prime} \iota_{p}\right)$. Specifically, we assume:

$$
\gamma_{\eta 0}=\left(\begin{array}{c}
\operatorname{Cov}\left(\eta_{i}, y_{i(1-p)}\right) \\
\vdots \\
\operatorname{Cov}\left(\eta_{i}, y_{i 0}\right)
\end{array}\right)=\frac{\sigma_{\eta}^{2}}{\left(1-\alpha^{\prime} \iota_{p}\right)} \iota_{p} . \quad \text { (Assumption B) }
$$

Under assumptions $A$ and $B$ the correlation between $y_{i t}$ and $\eta_{i}$ does not depend on $t$, so that the first differenced data are orthogonal to the effects. This situation led to orthogonality conditions for errors in levels used in the "system" GMM methods considered by Arellano and Bover (1995) and Blundell and Bond (1998). System GMM remained consistent in the presence of time series heteroskedasticity, and the random effects estimator discussed below can be regarded as a likelihoodbased counterpart to these procedures.

\subsection{Conditional Maximum Likelihood Estimation}

In order to construct a likelihood conditioned on the ML estimator of the effects under mean stationarity, we consider the following conditional normality assumption for $y_{i}^{0}$ given the effects:

$$
y_{i}^{0} \mid \mu_{i} \sim \mathcal{N}\left(\mu_{i} \iota_{p}, \Sigma_{00}\right)
$$

(Assumption G3) 
where $\Sigma_{00}$ satisfies $\Sigma_{00}=\Gamma_{00}-\iota_{p} \iota_{p}^{\prime} \sigma_{\eta}^{2} /\left(1-\alpha^{\prime} \iota_{p}\right)^{2}$.

Under assumptions $G 1$ and $G 3$

$$
y_{i}^{T} \mid \mu_{i} \sim \mathcal{N}\left(\mu_{i} \bar{L}, V\right)
$$

where $y_{i}^{T}=\left(y_{i}^{0 \prime}, y_{i 1}, \ldots, y_{i T}\right)^{\prime}, \tau$ denotes a vector of ones of order $(T+p)$, and

$$
V=\Gamma \Lambda^{\dagger} \Gamma^{\prime}
$$

with

$$
\Lambda^{\dagger}=\left(\begin{array}{cc}
\Sigma_{00} & 0 \\
0 & \Lambda
\end{array}\right), \quad \Gamma=\left(\begin{array}{cc}
I_{p} & 0 \\
-B_{T}^{-1} B_{T p} & B_{T}^{-1}
\end{array}\right)
$$

and

$$
B_{T p}=\left(\begin{array}{cccc}
-\alpha_{p} & -\alpha_{p-1} & \ldots & -\alpha_{1} \\
0 & -\alpha_{p} & \ldots & -\alpha_{2} \\
0 & 0 & \ddots & \\
\vdots & \vdots & & \\
0 & 0 & \ldots & 0
\end{array}\right)
$$

Thus

$$
\ln f\left(y_{i}^{T} \mid \mu_{i}\right)=-\frac{1}{2} \ln \operatorname{det} V-\frac{1}{2}\left(y_{i}^{T}-\mu_{i} \bar{\iota}\right)^{\prime} V^{-1}\left(y_{i}^{T}-\mu_{i} \bar{\iota}\right) .
$$

The MLE of $\mu_{i}$ for given $\alpha$ and $\Lambda^{\dagger}$ is

$$
\widehat{\mu}_{i}=\left(\bar{\iota}^{\prime} V^{-1} \bar{\iota}\right)^{-1} \bar{\iota}^{\prime} V^{-1} y_{i}^{T} .
$$

Next, to obtain the density of $y_{i}^{T}$ conditioned on $\widehat{\mu}_{i}$ (at true values of $\alpha$ and $\Lambda^{\dagger}$ ), it is simpler to use the transformation matrix

$$
\mathcal{H}=\left(\bar{\iota}^{\left(\bar{\iota}^{\prime} V^{-1} \bar{l}\right)^{-1} \bar{\iota}^{\prime} V^{-1}}\right)
$$

which transforms $y_{i}^{T}$ into $\left(\widehat{\mu}_{i}, \bar{D} y_{i}^{T}\right)$, where $\bar{D}$ denotes the $(T+p-1) \times(T+p)$ first-difference matrix operator. Since $y_{i}^{T} \mid \mu_{i}$ is normal so is $\mathcal{H} y_{i}^{T} \mid \mu_{i}$. Moreover,

$$
\operatorname{Var}\left(\mathcal{H} y_{i}^{T} \mid \mu_{i}\right)=\left(\begin{array}{cc}
\left(\bar{\iota}^{\prime} V^{-1} \bar{\iota}\right)^{-1} & 0 \\
0 & \bar{D} V \bar{D}^{\prime}
\end{array}\right)
$$

so that $\widehat{\mu}_{i}$ and $\bar{D} y_{i}^{T}$ are conditionally independent. Therefore,

$$
f\left(y_{i}^{T} \mid \mu_{i}\right)=f\left(\mathcal{H} y_{i}^{T} \mid \mu_{i}\right)|\operatorname{det} \mathcal{H}|=f\left(\bar{D} y_{i}^{T}\right) f\left(\widehat{\mu}_{i} \mid \mu_{i}\right) .
$$


This is so because $\bar{D} y_{i}^{T}$ is independent of $\mu_{i}$ and the fact that $|\operatorname{det} \mathcal{H}|=1$ (Arellano, 2003a, p. 94).

Therefore, the density of $y_{i}^{T}$ conditional on $\widehat{\mu}_{i}$ does not depend on $\mu_{i}$ and coincides with the density for the data in first differences:

$$
f\left(y_{i}^{T} \mid \widehat{\mu}_{i}, \mu_{i}\right)=\frac{f\left(y_{i}^{T} \mid \mu_{i}\right)}{f\left(\widehat{\mu}_{i} \mid \mu_{i}\right)}=f\left(\bar{D} y_{i}^{T}\right)
$$

which is

$$
\ln f\left(\bar{D} y_{i}^{T}\right)=-\frac{1}{2} \ln \operatorname{det}\left(\bar{D} V \bar{D}^{\prime}\right)-\frac{1}{2} y_{i}^{T \prime} \bar{D}^{\prime}\left(\bar{D} V \bar{D}^{\prime}\right)^{-1} \bar{D} y_{i}^{T} .
$$

This result is similar to the one discussed by Lancaster (2002) for a homoskedastic stationary model with $p=1$.

\section{Comparison with the Marginal Likelihood for First Differenced Data}

Thus, the log likelihood conditioned on the ML estimates of the effects under mean stationarity is a function of $\left(\alpha, \sigma_{1}^{2}, \ldots, \sigma_{T}^{2}\right.$, vech $\left.\Sigma_{00}\right)$ given by

$$
L_{C S}=-\frac{N}{2} \ln \operatorname{det}\left(\bar{D} V \bar{D}^{\prime}\right)-\frac{1}{2} \sum_{i=1}^{N} y_{i}^{T \prime} \bar{D}^{\prime}\left(\bar{D} V \bar{D}^{\prime}\right)^{-1} \bar{D} y_{i}^{T} .
$$

In the previous section we obtained a random effects likelihood (5.13) for data in first-differences without assuming mean stationarity as a function of $\left(\alpha, \sigma_{1}^{2}, \ldots, \sigma_{T}^{2}, \sigma_{\varepsilon}^{2 \dagger}, \phi^{\dagger}\right)$. This likelihood was conditioned on $\Delta y_{i}^{0}($ unless $p=1)$, but adding to it the likelihood of $\Delta y_{i}^{0}$, we can write the likelihood of $\bar{D} y_{i}^{T}$ in the absence of mean stationarity as a function of $\left(\alpha, \sigma_{1}^{2}, \ldots, \sigma_{T}^{2}, \sigma_{\varepsilon}^{2 \dagger}, \phi^{\dagger}\right)$ and $\Sigma_{\Delta}=\operatorname{Var}\left(\Delta y_{i}^{0}\right)$ given by $^{7}$

$$
L_{R D U}=L_{R D}-\frac{N}{2} \ln \operatorname{det} \Sigma_{\Delta}-\frac{1}{2} \operatorname{tr}\left(\Sigma_{\Delta}^{-1} Y_{\Delta}^{0 \prime} Y_{\Delta}^{0}\right) .
$$

If $p=1$ the likelihood of $\bar{D} y_{i}^{T}$ in the absence of mean stationarity is just the expression for $L_{R D}$ in (5.15).

In general, $\sigma_{\varepsilon}^{2 \dagger}$ satisfies expression (5.11), which under mean stationarity becomes ${ }^{8}$

$$
\sigma_{\varepsilon}^{2 \dagger}=\left(1-\alpha^{\prime} \iota_{p}\right)^{2} \sigma_{00}+\omega_{T}-\sigma_{10}^{\prime} D_{p}^{\prime}\left(D_{p} \Sigma_{00} D_{p}^{\prime}\right)^{-1} D_{p} \sigma_{10}
$$

\footnotetext{
${ }^{7}$ Note that $\Sigma_{\Delta}=D_{p} \Gamma_{00} D_{p}^{\prime}$ where $D_{p}$ is the first-difference operator of order $(p-1) \times p$.

${ }^{8}$ When $p=1$ we just have $\sigma_{00}=\Sigma_{00}$ and $\sigma_{\varepsilon}^{2 \dagger}=(1-\alpha)^{2} \sigma_{00}+\omega_{T}$.
} 
where we are using the partition of $\Sigma_{00}$

$$
\Sigma_{00}=\left(\begin{array}{cc}
\sigma_{00} & \sigma_{10}^{\prime} \\
\sigma_{10} & \Sigma_{11}
\end{array}\right)
$$

Similarly, under mean stationarity

$$
\phi^{\dagger}=\left(D_{p} \Sigma_{00} D_{p}^{\prime}\right)^{-1} D_{p} \sigma_{10} .
$$

However, both $\sigma_{\varepsilon}^{2 \dagger}$ and $\phi^{\dagger}$ remain free parameters because so is $\Sigma_{00}$.

Thus, the restriction of mean stationarity is immaterial to the data in first differences. $L_{R D U}$ and $L_{C S}$ are different parameterizations of the same criterion. Depending on ones taste it can be regarded as a mean-stationary conditional likelihood or as a nonstationary random effects likelihood for the first differenced data. In particular the estimator that maximizes $L_{C S}$ ( or $L_{R D}$ ) will be consistent under Assumption $A$ regardless of mean stationarity. ${ }^{9}$

Note that under homoskedasticity or covariance stationarity the situation is different because $\Sigma_{00}$ is no longer a matrix of free parameters, but tied to $\alpha$ and the common variance $\sigma^{2}$.

\subsection{Random Effects}

If in addition to assumptions $G 1$ and $G 3$ we assume that $\mu_{i}$ is normally distributed (as implied by $G 2$ ), we can obtain the integrated density marginal on $\mu_{i}$ :

$$
f\left(y_{i}^{T}\right)=\int f\left(y_{i}^{T} \mid \mu_{i}\right) d G\left(\mu_{i}\right)
$$

whose $\log$ is given by

$$
\ln f\left(y_{i}^{T}\right)=-\frac{1}{2} \ln \operatorname{det} \Omega-\frac{1}{2} y_{i}^{T \prime} \Omega^{-1} y_{i}^{T}
$$

with

$$
\Omega=\sigma_{\mu}^{2} \overline{\iota \iota}^{\prime}+V .
$$

Therefore, the random effects log likelihood under mean stationarity is a function of $\left(\alpha, \sigma_{1}^{2}, \ldots, \sigma_{T}^{2}, \operatorname{vech} \Sigma_{00}, \sigma_{\eta}^{2}\right)$ given by

$$
L_{R S}=-\frac{N}{2} \ln \operatorname{det} \Omega-\frac{1}{2} \sum_{i=1}^{N} y_{i}^{T \prime} \Omega^{-1} y_{i}^{T} .
$$

\footnotetext{
${ }^{9} \mathrm{~A}$ conceptual difference is that since $\sigma_{\varepsilon}^{2 \dagger}$ and $\phi^{\dagger}$ do not depend on $\sigma_{\eta}^{2}$ under mean stationarity, they would remain constant as $\sigma_{\eta}^{2} \rightarrow \infty$.
} 
The random effects ML estimator subject to mean stationarity (RML-s) maximizes $L_{R S}$ and is consistent and asymptotically normal under assumptions $A$ and $B$ regardless of non-normality or conditional heteroskedasticity.

In a three-wave panel with $p=1(T=2)$, the mean stationarity assumption imposes one restriction in the data covariance matrix $\Omega$, which corresponds to the orthogonality conditions for the system GMM estimator simulated in Arellano and Bover (1995):

$$
\begin{aligned}
E\left[y_{i 0}\left(\Delta y_{i 2}-\alpha \Delta y_{i 1}\right)\right] & =0 \\
E\left[\Delta y_{i 1}\left(y_{i 2}-\alpha y_{i 1}\right)\right] & =0 .
\end{aligned}
$$

RML-s provides a one-step estimator based on $T+1+p(p+3) / 2$ moment conditions that is asymptotically equivalent to two-step GMM system estimators under conditional homoskedasticity, and more efficient than standard one-step system estimators under time series heteroskedasticity.

As in the previous sections, the comparison between conditional and marginal ML estimates under stationarity can be understood as a straightforward comparison between covariance matrices of data in levels and first-differences

Relation to RML without Mean Stationarity Equation (4.3) in Section 4 gave the random effects log likelihood conditioned on $y_{i}^{0}$. Adding to this expression the likelihood of $y_{i}^{0}$, we can write the likelihood of $y_{i}^{T}$ in the absence of mean stationarity as a function of $\left(\alpha, \sigma_{1}^{2}, \ldots, \sigma_{T}^{2}, \phi, \sigma_{\varepsilon}^{2}, v e c h \Gamma_{00}\right)$ given by

$$
L_{R U}=L_{R}-\frac{N}{2} \ln \operatorname{det} \Gamma_{00}-\frac{1}{2} \operatorname{tr}\left(\Gamma_{00}^{-1} Y_{0}^{\prime} Y_{0}\right) .
$$

If $p=1$, in the parameterization of $L_{R U}$, mean stationarity can be expressed as the restriction

$$
\sigma_{\varepsilon}^{2}=(1-\alpha) \phi(1-\phi) \gamma_{00}+\omega_{T}
$$

Thus, RML-s can also be obtained maximizing $L_{R U}$ subject to (6.22) in that case.

\section{Unit Roots}

In this section we discuss the possibility of identification failure when the autoregressive process has a unit root. We focus on the $p=1$ case, so that the unit root 
model is

$$
y_{i t}=t \eta_{i}+v_{i t}+v_{i(t-1)}+\ldots+v_{i 1}+y_{i 0} .
$$

For this process, if $\sigma_{\eta}^{2}=0$ the rank condition for GMM based on lagged levels as instruments for errors in differences fails, because changes in $y_{i t}$ are uncorrelated to lagged levels (e.g. Arellano and Honoré, 2001). ${ }^{10}$ Thus, $\alpha$ would not be identified from RML in a three-wave panel $(T=2)$ when the true value is one, since in that case RML coincides with the IV estimator based on

$$
E\left[y_{i 0}\left(\Delta y_{i 2}-\alpha \Delta y_{i 1}\right)\right]=0
$$

Since the estimating criteria for the previous estimators depend on the data exclusively through second moments, it is useful to look at the restrictions implied by the model on the data covariance matrix. Following Ahn and Schmidt (1995), for $T \geq 3$ these restrictions can be represented as

$$
\begin{gathered}
E\left[y_{i s}\left(\Delta y_{i t}-\alpha \Delta y_{i(t-1)}\right)\right]=0 \quad(t=2, \ldots, T ; s=0, \ldots, t-2) \\
E\left[\left(\Delta y_{i(t-1)}-\alpha \Delta y_{i(t-2)}\right)\left(y_{i t}-\alpha y_{i(t-1)}\right)\right]=0 \quad(t=3, \ldots, T) .
\end{gathered}
$$

When $T=3$ and the true values are $\bar{\alpha}=1$ and $\bar{\sigma}_{\eta}^{2}=0,(7.2)$ consists of just one quadratic equation

$$
a_{1} \alpha^{2}+b_{1} \alpha+c_{1}=0
$$

with coefficients given by

$$
\begin{aligned}
a_{1} & =E\left(y_{i 2} \Delta y_{i 1}\right)=\bar{\sigma}_{1}^{2} \\
b_{1} & =-E\left(y_{i 2} \Delta y_{i 2}+y_{i 3} \Delta y_{i 1}\right)=-\left(\bar{\sigma}_{1}^{2}+\bar{\sigma}_{2}^{2}\right) \\
c_{1} & =E\left(y_{i 3} \Delta y_{i 2}\right)=\bar{\sigma}_{2}^{2}
\end{aligned}
$$

where $\bar{\sigma}_{1}^{2}, \bar{\sigma}_{2}^{2}$ and $\bar{\sigma}_{3}^{2}$ denote the true values of the error variances.

Equation (7.3) has two roots given by

$$
\frac{\bar{\sigma}_{1}^{2}+\bar{\sigma}_{2}^{2} \pm\left(\bar{\sigma}_{2}^{2}-\bar{\sigma}_{1}^{2}\right)}{2 \bar{\sigma}_{1}^{2}}=\left\{\begin{array}{l}
\alpha_{1}^{*}=\bar{\sigma}_{2}^{2} / \bar{\sigma}_{1}^{2} \\
\bar{\alpha}=1
\end{array}\right.
$$

\footnotetext{
${ }^{10}$ When $\alpha=1$ and $\sigma_{\eta}^{2}=0$, heterogeneity only plays a role in the determination of the initial observations of the process. In contrast, if $\sigma_{\eta}^{2} \neq 0$ the model is a random walk with heterogeneous linear growth.
} 
Therefore, under time series heteroskedasticity there is local identification from (7.3) but not global identification. If $\bar{\sigma}_{1}^{2}=\bar{\sigma}_{2}^{2}$ there is global identification but first-order underidentification, because the first derivative of (7.3)

$$
2 a_{1} \alpha+b_{1}=0
$$

vanishes at $\alpha=1$. In that case there is second-order identification because $\alpha=1$ is the only solution to equation (7.5) and the second derivative does not vanish (Sargan, 1983).

In general, we get $T-2$ equations of the same form as (7.3), each one with a solution of the form $\alpha_{t}^{*}=\bar{\sigma}_{t+1}^{2} / \bar{\sigma}_{t}^{2}$, aside from unity. Thus, for $T>3$ there is both first-order and global identification from (7.2) under heteroskedasticity, unless the unconditional variances change at a constant rate of growth (i.e. $\bar{\sigma}_{t+1}^{2} / \bar{\sigma}_{t}^{2}$ is constant for $t=1, \ldots, T-2)$.

Heteroskedastic BCS and Unit Roots Next, we develop the local identification result for the bias-corrected CML scores when $T=3$. The expected BCS equations are given by

$$
\begin{gathered}
E\left[x_{i}^{\prime} D^{\prime}\left(D \Lambda D^{\prime}\right)^{-1} D v_{i}\right]=-h_{T}(\alpha, \varphi) \\
E\left[K^{\prime}\left(D \Lambda D^{\prime} \otimes D \Lambda D^{\prime}\right)^{-1} \operatorname{vec}\left(D v_{i} v_{i}^{\prime} D^{\prime}-D \Lambda D^{\prime}\right)\right]=0 .
\end{gathered}
$$

where

$$
\begin{gathered}
D x_{i}=\left(\begin{array}{c}
\Delta y_{i 1} \\
\Delta y_{i 2}
\end{array}\right), \quad D y_{i}=\left(\begin{array}{c}
\Delta y_{i 2} \\
\Delta y_{i 3}
\end{array}\right) \\
\left(D \Lambda D^{\prime}\right)^{-1}=\frac{1}{\left(\sigma_{1}^{2} \sigma_{2}^{2}+\sigma_{1}^{2} \sigma_{3}^{2}+\sigma_{2}^{2} \sigma_{3}^{2}\right)}\left(\begin{array}{cc}
\sigma_{2}^{2}+\sigma_{3}^{2} & \sigma_{2}^{2} \\
\sigma_{2}^{2} & \sigma_{1}^{2}+\sigma_{2}^{2}
\end{array}\right),
\end{gathered}
$$

and

$$
h_{T}(\alpha, \varphi)=\varphi_{2}+(1+\alpha) \varphi_{3}=\frac{\sigma_{1}^{2}}{\left(\sigma_{1}^{2} \sigma_{2}^{2}+\sigma_{1}^{2} \sigma_{3}^{2}+\sigma_{2}^{2} \sigma_{3}^{2}\right)}\left[\sigma_{3}^{2}+(1+\alpha) \sigma_{2}^{2}\right] .
$$

When the true values are $\bar{\alpha}=1$ and $\bar{\sigma}_{\eta}^{2}=0$, the first score (7.6) can be written as

$$
\operatorname{tr}\left[\left(\begin{array}{cc}
\sigma_{2}^{2}+\sigma_{3}^{2} & \sigma_{2}^{2} \\
\sigma_{2}^{2} & \sigma_{1}^{2}+\sigma_{2}^{2}
\end{array}\right)\left(\begin{array}{cc}
\alpha \bar{\sigma}_{1}^{2} & -\bar{\sigma}_{2}^{2} \\
0 & \alpha \bar{\sigma}_{2}^{2}
\end{array}\right)\right]=\sigma_{1}^{2}\left[\left(\sigma_{2}^{2}+\sigma_{3}^{2}\right)+\alpha \sigma_{2}^{2}\right]
$$


Moreover,

$$
E\left(D v_{i} v_{i}^{\prime} D^{\prime}\right)=\left(\begin{array}{cc}
\bar{\sigma}_{2}^{2}+\alpha^{2} \bar{\sigma}_{1}^{2} & -\alpha \bar{\sigma}_{2}^{2} \\
-\alpha \bar{\sigma}_{2}^{2} & \bar{\sigma}_{3}^{2}+\alpha^{2} \bar{\sigma}_{2}^{2}
\end{array}\right)
$$

Hence, in view of the second block of scores (7.7), we have

$$
\begin{aligned}
\bar{\sigma}_{2}^{2}+\alpha^{2} \bar{\sigma}_{1}^{2} & =\sigma_{1}^{2}+\sigma_{2}^{2} \\
\alpha \bar{\sigma}_{2}^{2} & =\sigma_{2}^{2} \\
\bar{\sigma}_{3}^{2}+\alpha^{2} \bar{\sigma}_{2}^{2} & =\sigma_{2}^{2}+\sigma_{3}^{2}
\end{aligned}
$$

Now, substituting in (7.8)

$\operatorname{tr}\left[\left(\begin{array}{cc}\bar{\sigma}_{3}^{2}+\alpha^{2} \bar{\sigma}_{2}^{2} & \alpha \bar{\sigma}_{2}^{2} \\ \alpha \bar{\sigma}_{2}^{2} & \bar{\sigma}_{2}^{2}+\alpha^{2} \bar{\sigma}_{1}^{2}\end{array}\right)\left(\begin{array}{cc}\alpha \bar{\sigma}_{1}^{2} & -\bar{\sigma}_{2}^{2} \\ 0 & \alpha \bar{\sigma}_{2}^{2}\end{array}\right)\right]=\left(\bar{\sigma}_{2}^{2}+\alpha^{2} \bar{\sigma}_{1}^{2}-\alpha \bar{\sigma}_{2}^{2}\right)\left(\bar{\sigma}_{3}^{2}+2 \alpha^{2} \bar{\sigma}_{2}^{2}\right)$,

which can be rearranged as

$$
(1-\alpha)\left(\bar{\sigma}_{2}^{2}-\bar{\sigma}_{1}^{2} \alpha\right)\left(\bar{\sigma}_{3}^{2}+2 \bar{\sigma}_{2}^{2} \alpha^{2}\right)=0 .
$$

Thus, as before there are two real roots: $\bar{\alpha}=1$ and $\alpha^{*}=\bar{\sigma}_{2}^{2} / \bar{\sigma}_{1}^{2}$. Corresponding to $\alpha=1$ we have

$$
\left(\begin{array}{l}
\sigma_{1}^{2} \\
\sigma_{2}^{2} \\
\sigma_{3}^{2}
\end{array}\right)=\left(\begin{array}{c}
\bar{\sigma}_{1}^{2} \\
\bar{\sigma}_{2}^{2} \\
\bar{\sigma}_{3}^{2}
\end{array}\right),
$$

and corresponding to $\alpha=\bar{\sigma}_{2}^{2} / \bar{\sigma}_{1}^{2}$

$$
\left(\begin{array}{c}
\sigma_{1}^{2} \\
\sigma_{2}^{2} \\
\sigma_{3}^{2}
\end{array}\right)=\left(\begin{array}{c}
\sigma_{1}^{2 *} \\
\sigma_{2}^{2 *} \\
\sigma_{3}^{2 *}
\end{array}\right) \equiv\left(\begin{array}{c}
\bar{\sigma}_{2}^{2} \\
\bar{\sigma}_{2}^{4} \\
\bar{\sigma}_{1}^{2} \\
\bar{\sigma}_{3}^{2}-\frac{\bar{\sigma}_{2}^{4}}{\bar{\sigma}_{1}^{4}}\left(\bar{\sigma}_{1}^{2}-\bar{\sigma}_{2}^{2}\right)
\end{array}\right) .
$$

Expected RML Likelihood Finally, we consider the expected random effects likelihood for one observation when $T=3, \bar{\alpha}=1$ and $\bar{\sigma}_{\eta}^{2}=0$. This is a function of $\left(\alpha, \sigma_{1}^{2}, \sigma_{2}^{2}, \sigma_{3}^{2}, \phi, \sigma_{\varepsilon}^{2}\right)$ given by

$$
\begin{aligned}
E\left(L_{R i}\right)= & -\frac{1}{2} \ln \operatorname{det}\left(D \Lambda D^{\prime}\right)-\frac{1}{2} \operatorname{tr}\left[\left(D \Lambda D^{\prime}\right)^{-1} E\left(D v_{i} v_{i}^{\prime} D^{\prime}\right)\right] \\
& -\frac{1}{2} \ln \sigma_{\varepsilon}^{2}-\frac{1}{2 \sigma_{\varepsilon}^{2}} E\left[\left(\bar{u}_{i}-\phi y_{i 0}\right)^{2}\right]
\end{aligned}
$$

Note that the true values of $\phi$ and $\sigma_{\varepsilon}^{2}$ are $\bar{\phi}=0$ and $\bar{\sigma}_{\varepsilon}^{2}=\bar{\omega}_{T}$. 
Maximizing $E\left(L_{R i}\right)$ with respect to $\phi, \sigma_{\varepsilon}^{2}$ for given $\left(\alpha, \sigma_{1}^{2}, \sigma_{2}^{2}, \sigma_{3}^{2}\right)$ we get

$$
\begin{gathered}
\phi=1-\alpha \\
\sigma_{\varepsilon}^{2}=E\left[\left(\bar{y}_{i}-\alpha \bar{x}_{i}-(1-\alpha) y_{i 0}\right)^{2}\right]=E\left[\left(\bar{y}_{i}^{\dagger}-\alpha \bar{x}_{i}^{\dagger}\right)^{2}\right] .
\end{gathered}
$$

Therefore, the concentrated expected likelihood for the data in levels and in differences coincide. An implication is that when $\bar{\alpha}=1$ and $\bar{\sigma}_{\eta}^{2}=0$, RML in levels and RML in differences are asymptotically equivalent.

Moreover, the maximum of $E\left(L_{R i}\right)$ is attained at

$$
\max E\left(L_{R i}\right)=-\frac{1}{2} \ln \left(\bar{\sigma}_{1}^{2} \bar{\sigma}_{2}^{2} \bar{\sigma}_{3}^{2}\right)-\frac{3}{2}
$$

by $\left(\bar{\alpha}, \bar{\sigma}_{1}^{2}, \bar{\sigma}_{2}^{2}, \bar{\sigma}_{3}^{2}, \bar{\phi}, \bar{\sigma}_{\varepsilon}^{2}\right)$ and $\left(\alpha^{*}, \sigma_{1}^{2 *}, \sigma_{2}^{2 *}, \sigma_{3}^{2 *}, \phi^{*}, \sigma_{\varepsilon}^{2 *}\right)$, where

$$
\begin{aligned}
\phi^{*} & =1-\alpha^{*}=1-\frac{\bar{\sigma}_{2}^{2}}{\bar{\sigma}_{1}^{2}} \\
\sigma_{\varepsilon}^{2 *} & =\frac{\bar{\sigma}_{1}^{2} \bar{\sigma}_{2}^{2} \bar{\sigma}_{3}^{2}}{\bar{\sigma}_{2}^{2} \bar{\sigma}_{3}^{2}+\bar{\sigma}_{2}^{4} \bar{\sigma}_{3}^{2}}+\frac{\bar{\sigma}_{2}^{10}}{\bar{\sigma}_{1}^{6}}
\end{aligned}
$$

which completes the characterization of the two observationally equivalent points.

\section{Calculations of Relative Asymptotic Variances}

We perform numerical calculations of the asymptotic variances for various estimators of the autoregressive coefficient. We report, for $p=1$, the asymptotic variances of both homoskedastic and heteroskedastic BCS and RML-dif estimators, relative to the corresponding RML in levels, calculated under the assumption of normality. Formulae for the asymptotic variances are derived in Appendix B.

The interest of the exercise is in providing information on the efficiency gains that can be expected from the levels of the data, relative to only using firstdifferences, when RML is the maximum likelihood estimator, and stationarity restrictions are not enforced. In addition, we also get to know about the magnitude of the asymptotic inefficiency of BCS relative to RML-dif under normality.

Figures 1 and 2 show values of the asymptotic standard deviations of the homoskedastic BCS and RML-dif estimators relative to the standard deviation 
of RML, for non-negative values of $\alpha$. The calculations are for $T=2,3$, and 9, under stationarity and homoskedasticity with $\sigma^{2}=1$. $^{11}$

The $T=2$ case is special because in that situation BCS and RML-dif coincide and their ability to identify $\alpha$ rests exclusively on the homoskedasticity restriction.

In Figure 1 the variance of the effects has been set to zero $\left(\lambda=\sigma_{\eta}^{2} / \sigma^{2}=0\right)$, whereas in Figure $2 \sigma_{\eta}^{2}$ and $\sigma^{2}$ are equal $(\lambda=1)$. The relative inefficiency of both estimators increases monotonically with $\alpha$ and decreases with $\lambda$ and T. Figure 1 shows potentially important efficiency gains from using the levels when $T=3$ and $\alpha$ is large, but the gains become much smaller when $\lambda=1$, as shown in Figure 2.

In Figure 3 we explore the impact of nonstationarity. We calculate the same relative inefficiency measures as in the previous figures for different values of the ratio of the actual to the steady state standard deviations of $y_{0}$. Thus, under stationarity $\kappa=1$, and a value of $\kappa=2$ means that the standard deviation of initial conditions is twice the standard deviation of the steady state standard deviation of the process. We set $T=3, \lambda=0$, and $\alpha=0.9$, so that we essentially calculate the maximal inefficiencies for each value of $\kappa$. For $\kappa<1$, the inefficiency of BCS can be enormous, whereas the inefficiency of RML-dif is much smaller and shows a non-monotonic pattern.

Turning to heteroskedastic estimators, Figures 4 and 5 display relative inefficiency ratios for heteroskedastic BCS and RML-dif, similar to those in the previous figures. The calculations are under homoskedasticity and stationarity, for $\lambda=0$ and $1, T=3$ and 9 , and $\sigma^{2}=1$. As before, the inefficiencies of heteroskedastic BCS and RML-dif increase with $\alpha$ and decrease with $\lambda$ and $T$, but they have larger magnitudes than those of their homoskedastic counterparts.

Table 1 illustrates the extent of these differences by showing the inefficiencies of homoskedastic and heteroskedastic estimators for selected values of the parameters. Some of the inefficiencies are quite large. For example, for the heteroskedastic estimators with $\alpha=0.8, T^{\circ}=4$ and $\lambda=0$, the standard error of RML-dif is more than twice that of RML-lev, and the standard error of BCS is more than three times as large.

Finally, Figure 6 reports asymptotic standard deviations of BCS and RML when $\alpha=1$ and $\lambda=0$ (in this case RML-dif and RML-lev are asymptotically equivalent) for $T=6$ and a single break in the error variance. Standard deviations

\footnotetext{
${ }^{11}$ Because of stationarity $\gamma_{00}=\sigma^{2} /\left(1-\alpha^{2}\right)$, so that it increases with $\alpha$.
} 
(scaled by $\sqrt{900}$ ) are given as a function of the percentage change in variance and for two different locations of the variance break (which takes place either during the last 2 or the last 4 periods). ${ }^{12}$ As expected, asymptotic standard deviations decrease with the strength of heteroskedasticity, and are smaller when the variance break is centrally located than when it only occurs during the last two periods.

\section{Empirical Illustration: Individual Earnings Dynamics}

In order to illustrate the properties of the previous methods, we estimate firstand second-order autoregressive equations for individual labour income using two different samples. The first one is a sample of Spanish men from the European Community Household Panel (ECHP) for the period 1994-1999. The second is a sample from PSID for the period 1977-1983 taken from Alvarez, Browning, and Ejrnæs (2001).

There are 632 individuals in the Spanish data set and 792 in the PSID sample. All individuals in both data sets are married males, who are aged 20-65 during the sample period, heads of household, and continuously employed. The earnings variable is similarly defined in the two samples as total annual labour income of the head.

The variables that we use in the estimation are log earnings residuals from firststage regressions on age, age squared, education and year dummies (see Alvarez, Browning, and Ejrnæs, 2001, for further details on the PSID sample, and tables A1 and A2 for the Spanish sample). Log earnings have a much higher variance in the PSID sample than in the Spanish one. Moreover, the PSID data show a sharp rise in the variance of earnings in 1982 (a widely documented fact), whereas there is no appreciable change in the variance in the Spanish sample during the (different) years that we observe.

The AR(1) results for the Spanish data are reported in the first part of Table 2. Heteroskedastic bias-corrected score (BCS) and random effects (RMLr) estimates of the autoregressive coefficient are very similar. They are also very close to the homoskedastic random effects estimate (RMLnr), which is not surprising given the absence of change in the period-specific variance estimates reported in the table.

\footnotetext{
${ }^{12}$ When $\alpha=1$, we considered choices of $\gamma_{00}$ and $\gamma_{\eta 0}$ of the form $\gamma_{00}=\kappa \bar{\sigma}^{2}+\kappa^{2} \sigma_{\eta}^{2}$ and $\gamma_{\eta 0}=\kappa \sigma_{\eta}^{2}$, where $\bar{\sigma}^{2}=T^{-1} \sum_{t=1}^{T} \sigma_{t}^{2}$. But for the calculations in Figure 6 , since $\sigma_{\eta}^{2}=0$ the results turn out to be invariant to the choice of $\kappa$.
} 
By comparison, the AR(1) GMM estimates (one- and two-step) are very small, given that GMM, BCS, and RMLr are all consistent under similar assumptions. The system GMM estimator, that relies on mean stationarity, is more in line with the likelihood-based estimates, although probably for the wrong reasons, given the rejection of mean stationarity that is apparent from the Sargan test. The RMLr estimate subject to mean stationarity is smaller than system-GMM, but a Wald test of the mean stationarity restriction rejects (with a " $t$ ratio" for $\sigma_{\varepsilon}^{2}$ of 2.54). Finally, within-groups (WG) and the random effects estimate that rules out correlation between the effects and initial observations (RML, $\phi=0$ ) exhibit, respectively, the downward and upward biases that would be predicted from theory.

The AR(1) results for the PSID sample, reported in Table 3, also show a marked discrepancy between the likelihood-based estimates and GMM, and a similar rejection of mean stationarity from the incremental Sargan test, although not from RML estimation (the " $t$ ratio" for $\sigma_{\varepsilon}^{2}$ is just 0.16 ). In the PSID data there is more state dependence than in the Spanish data, at least as measured by the first-order autoregressive coefficient. There is also more variation in the errors and substantial time series heteroskedasticity. The latter translate into a small but noticeable upward bias in the RML estimate calculated under the assumption of homoskedasticity.

Given the $\mathrm{AR}(1)$ estimates reported in the tables, the variance of the effects can be recovered from $\sigma_{\eta}^{2}=\sigma_{\varepsilon}^{2}+\phi^{2} \gamma_{00}-\omega_{T}$ (as explained in Section 4), which gives $\widehat{\sigma}_{\eta}^{2}=0.05$ for the Spanish data, and $\widehat{\sigma}_{\eta}^{2}=0.07$ for the PSID.

GMM estimates are known to be downward biased in finite samples, specially when the number of moments is large and the instruments are weak. However, the magnitude of the bias in our application (relative to the likelihood estimates) is puzzling for the values of $\alpha$ and $T / N$ that we have, suggesting misspecification as the most likely reason for these discrepancies. This impression is confirmed by the $\operatorname{AR}(2)$ estimates and the simulation results reported below.

The upshot from the $\operatorname{AR}(2)$ estimates reported in the second parts of tables 2 and 3 is that there is a positive autoregressive root, in the $(0.4,0.5)$ range for the Spanish panel and in the $(0.6,0.7)$ range for PSID, and a negative root of around -0.2 in both datasets (so that an $\operatorname{ARMA}(1,1)$ model would provide a similar fit).

$\operatorname{AR}(2)$ GMM estimates are still smaller than the likelihood-based estimates, 
and there is a discrepancy between BCS and RMLr (specially for PSID), all of which suggests that there may be some remaining misspecification. ${ }^{13}$ Mean stationarity is rejected in both datasets and, when enforced, leads to somewhat larger positive roots.

However, in contrast with other studies that either imposed or found a unit root in individual earnings (e.g. MaCurdy, 1982), we find no evidence of unit roots. The only way we managed to obtain a near-unit root is by imposing the restriction that the initial observations of earnings are orthogonal to the unobserved component (i.e. $\phi=0$ ). Doing this led to an estimated positive root of 0.95 in both panels. Clearly, if only heterogeneity that is orthogonal to initial observations is allowed, any nonorthogonal heterogeneity will be captured by the autoregressive part of the model as spurious state dependence.

Moving Average Errors We checked whether this conclusion was affected by adding a moving average component to the specification of PSID earnings. In such a case the autoregressive coefficients can no longer be interpreted as a model for the conditional expectation of earnings given past observations, but an ARMA model might lead to a more parsimonious specification. Moreover, models of earnings that specify a measurement error component imply a reduced form with moving average errors. Appendix D describes our ARMA specification and the random effects ML estimators that we used.

Table 4 reports $\operatorname{ARMA}(1,1), \operatorname{ARMA}(1,2)$, and $\operatorname{ARMA}(2,1)$ estimates from the PSID sample. As expected, the ARMA(1,1) estimates are similar to those obtained from the $\operatorname{AR}(2)$ specification. However, the $\operatorname{ARMA}(1,2)$ and the $\operatorname{ARMA}(2,1)$ estimates were very imprecise, suggesting that there is no enough variation in the data covariance matrix to support a three-parameter dynamic specification within this class of models.

Testing for Nonnormality The distributions of the effects and the autoregressive errors are nonparametrically identified and can be estimated using deconvolution techniques as in Horowitz and Markatou (1996).

Horowitz and Markatou carried out graphical tests of normality of the distri-

\footnotetext{
${ }^{13}$ We found that the BCS equations, in addition to the stable solution, had another solution with an explosive root.
} 
butions of errors and effects in a static earnings model using a two-wave panel from the CPS. ${ }^{14}$ We used their diagnostics and found very similar results for PSID autoregressive models. A normal probability plot of residuals in first-differences (Figure 7) indicates that the tails of the distribution of errors are thicker than those of the normal distribution. However, a plot of the log empirical characteristic function of the effects against minus the square of its argument is almost a straight line, hence showing no deviation from normality (Figure 8).

Monte Carlo Simulations To illustrate the properties of the estimators, we performed a small simulation exercise calibrated to the likelihood-based AR(1) estimates from PSID data. We generated 1000 replications with $N=792, T^{\circ}=7$, $\eta_{i} \sim \mathcal{N}\left(0, \sigma_{\eta}^{2}\right), v_{i t} \sim \mathcal{N}\left(0, \sigma_{t}^{2}\right), \sigma_{\eta}^{2}=0.07$, and mean stationarity.

In Table 5 we report means and standard deviations of the WG, GMM1, $\mathrm{RML}(\mathrm{nr}), \mathrm{RML}(\mathrm{r})$, and BCS estimators of the $\mathrm{AR}(1)$ model for $\alpha=0.4$ and 0.8 (with $\bar{\sigma}_{0}^{2}=0.11$ and 0.28 , respectively). The results show that both RML(r) and BCS are virtually unbiased. Those for $\alpha=0.4$ nicely reproduce the WG downward bias and the RML(nr) upward bias that we found in the PSID sample. However, the results fail to explain the performance of GMM with the real data, which reinforces the evidence of misspecification in the $\mathrm{AR}(1)$ earnings models.

\section{Concluding Remarks}

From a GMM perspective, a motivation for considering likelihood based estimators is to reduce the number of moments available for estimation. The number of orthogonality conditions of optimal GMM estimators in autoregressive panel models grows at a rate of $T(T-1) / 2$, whereas the number of score equations for the heteroskedastic likelihood estimators grows at a rate of $T$. An interesting question is to characterize the potential incidental parameter problem that occurs for these estimators as $T$ tends to infinity.

From ongoing work by the authors, we conjecture that in a double asymptotic setup where $T / N$ tends to a finite constant, the estimators with unrestricted time series variances remain consistent and asymptotically normal, but have a bias term in the asymptotic distribution when the data are not symmetrically distributed.

\footnotetext{
${ }^{14}$ Figures 1 and 5 in their paper
} 


\section{References}

Abowd, J.M. and D. Card (1989): "On the Covariance Structure of Earnings and Hours Changes", Econometrica, 57, 411-445.

Ahn, S. and P. Schmidt (1995): "Efficient Estimation of Models for Dynamic Panel Data", Journal of Econometrics, 68, 5-27.

Alvarez, J. and M. Arellano (2003): "The Time Series and Cross-Section Asymptotics of Dynamic Panel Data Estimators", Econometrica, 71, 1121-1159.

Alvarez, J., M. Browning, and M. Ejrnæs (2001): "Modelling Income Processes with Lots of Heterogeneity", University of Copenhagen, unpublished.

Anderson, T. W. and C. Hsiao (1981): "Estimation of Dynamic Models with Error Components", Journal of the American Statistical Association, 76, 598-606.

Arellano, M. and S. R. Bond (1991): "Some Tests of Specification for Panel Data: Monte Carlo Evidence and an Application to Employment Equations", Review of Economic Studies, 58, 277-297.

Arellano, M. and O. Bover (1995): "Another Look at the Instrumental-Variable Estimation of Error-Components Models", Journal of Econometrics, 68, 2951.

Arellano, M. and B. Honoré (2001): "Panel Data Models: Some Recent Developments", in J. J. Heckman and E. Leamer (eds.), Handbook of Econometrics, vol. 5, chapter 53, North-Holland.

Arellano, M. (2003a): Panel Data Econometrics, Oxford University Press, Oxford.

Arellano, M. (2003b): "Discrete Choices with Panel Data", Investigaciones Económicas, 27, 423-458.

Barro, R. J. and X. Sala-i-Martin (1995): Economic Growth, McGraw-Hill, New York.

Blundell, R. and S. Bond (1998): "Initial Conditions and Moment Restrictions in Dynamic Panel Data Models", Journal of Econometrics, 87, 115-143.

Blundell, R. and R. Smith (1991), "Initial Conditions and Efficient Estimation in Dynamic Panel Data Models", Annales d'Economie et de Statistique, 20/21, 109-123.

Chamberlain, G. (1980): "Analysis of Covariance with Qualitative Data", Reiew of Economic Studies, 47, 225-238. 
Cox, D. R. and N. Reid (1987): "Parameter Orthogonality and Approximate Conditional Inference" (with discussion), Journal of the Royal Statistical Society, Series B, 49, 1-39.

Gourieroux, C., A. Monfort, and A. Trognon (1984): "Pseudo Maximum Likelihood Methods: Theory", Econometrica, 52, 681-700.

Hahn, J. and G. Kuersteiner (2002): "Asymptotically Unbiased Inference for a Dynamic Panel Model with Fixed Effects When Both $n$ and $T$ are Large", Econometrica, 70, 1639-1657.

Hall, R. and F. Mishkin (1982): "The Sensitivity of Consumption to Transitory Income: Estimates from Panel Data on Households", Econometrica, 50, 461-481.

Hause, J. C. (1980): "The Fine Structure of Earnings and the On-the-Job Training Hypothesis", Econometrica, 48, 1013-1029.

Holtz-Eakin, D., W. Newey, and H. Rosen (1988): "Estimating Vector Autoregressions with Panel Data", Econometrica, 56, 1371-1395.

Horowitz, J. L. and M. Markatou (1996): "Semiparametric Estimation of Regression Models for Panel Data", Review of Economic Studies, 63, 145-168.

Hsiao, C., M. H. Pesaran, and A. K. Tahmiscioglu (2002): "Maximum Likelihood Estimation of Fixed Effects Dynamic Panel Data Models Covering Short Time Periods", Journal of Econometrics, 109, 107-150.

Kiviet, J.F. (1995), "On Bias, Inconsistency, and Efficiency of Various Estimators in Dynamic Panel Data Models", Journal of Econometrics, 68, 53-78.

Lancaster, T. (2002): "Orthogonal Parameters and Panel Data", Review of Economic Studies, 69, 647-666.

MaCurdy, T. E. (1982): "The Use of Time Series Processes to Model the Error Structure of Earnings in a Longitudinal Data Analysis", Journal of Econometrics, 18, 83-114.

Meghir, C. and L. Pistaferri (2004): "Income Variance Dynamics and Heterogeneity", Econometrica, 72, 1-32.

Nickell, S. (1981): "Biases in Dynamic Models with Fixed Effects", Econometrica, 49, 1417-1426.

Sargan, J. D. (1983): "Identification and Lack of Identification", Econometrica, $51,1605-1634$.

Sims, C. A. (2000): "Using a Likelihood Perspective to Sharpen Econometric Discourse: Three Examples", Journal of Econometrics, 95, 443-462. 
Table 1

Relative Inefficiency Ratios*

\begin{tabular}{|c|c|c|c|c|c|}
\hline & & \multicolumn{2}{|c|}{ Homosk. } & \multicolumn{2}{|c|}{ Heterosk. } \\
\hline & & BCS & RMLdif & BCS & RMLdif \\
\hline & & & $\alpha=0.6$ & & \\
\hline \multirow[t]{2}{*}{$T^{o}=4$} & $\lambda=0$ & 1.45 & 1.33 & 2.21 & 1.59 \\
\hline & $\lambda=1$ & 1.14 & 1.05 & 1.56 & 1.12 \\
\hline \multirow[t]{2}{*}{$T^{o}=10$} & $\lambda=0$ & 1.06 & 1.04 & 1.07 & 1.05 \\
\hline & $\lambda=1$ & 1.02 & 1.00 & 1.03 & 1.00 \\
\hline \multirow{3}{*}{$T^{o}=4$} & & & $\alpha=0.8$ & & \\
\hline & $\lambda=0$ & 1.93 & 1.70 & 3.16 & 2.15 \\
\hline & $\lambda=1$ & 1.22 & 1.07 & 1.69 & 1.15 \\
\hline \multirow[t]{2}{*}{$T^{o}=10$} & $\lambda=0$ & 1.22 & 1.13 & 1.28 & 1.15 \\
\hline & $\lambda=1$ & 1.08 & 1.01 & 1.12 & 1.01 \\
\hline
\end{tabular}


Table 2

Autoregressive Model of Earnings

AR(1) Estimates for Spanish Data, 1994-1999

$N=632, T^{0}=6$

\begin{tabular}{|c|c|c|c|c|c|}
\hline & WG & GMM1 & GMM2 & System-GMM & \\
\hline \multirow[t]{2}{*}{$\alpha$} & -0.022 & 0.042 & 0.038 & 0.183 & \\
\hline & $(-0.95)$ & $(0.93)$ & $(0.87)$ & $(7.00)$ & \\
\hline Sargan test (df) & & & $6.11(9)$ & $22.71(13)$ & \\
\hline$m 1$ & & -9.67 & -9.89 & -13.73 & \\
\hline \multirow[t]{4}{*}{$m 2$} & & 0.27 & 0.23 & 1.83 & \\
\hline & Lik & lihood-ba & ed Estimat & & \\
\hline & BCS & $\mathrm{RML}(\mathrm{r})$ & RML(nr) & RML(r) & $\mathrm{RML}(\mathrm{r})$ \\
\hline & (robust) & (robust) & (homosk.) & (mean stat.) & $(\phi=0)$ \\
\hline \multirow[t]{2}{*}{$\alpha$} & 0.218 & 0.200 & 0.207 & 0.164 & 0.926 \\
\hline & $(7.04)$ & $(7.07)$ & $(3.83)$ & $(5.32)$ & $(87.05)$ \\
\hline \multirow[t]{2}{*}{$\sigma_{1}^{2}(1995)$} & 0.025 & 0.023 & 0.023 & 0.023 & 0.049 \\
\hline & $(11.34)$ & $(11.91)$ & $(25.14)$ & $(11.65)$ & $(12.81)$ \\
\hline \multirow[t]{2}{*}{$\sigma_{2}^{2}(1996)$} & 0.022 & 0.021 & & 0.021 & 0.042 \\
\hline & $(8.55)$ & $(9.28)$ & & $(9.04)$ & $(14.40)$ \\
\hline \multirow[t]{2}{*}{$\sigma_{3}^{2}(1997)$} & 0.023 & 0.023 & & 0.023 & 0.039 \\
\hline & $(8.23)$ & $(9.55)$ & & $(9.16)$ & $(15.96)$ \\
\hline \multirow[t]{2}{*}{$\sigma_{4}^{2}(1998)$} & 0.023 & 0.023 & & 0.022 & 0.039 \\
\hline & $(10.26)$ & $(10.60)$ & & $(10.47)$ & $(14.74)$ \\
\hline \multirow[t]{2}{*}{$\sigma_{5}^{2}(1999)$} & 0.023 & 0.025 & & 0.025 & 0.047 \\
\hline & $(10.93)$ & (11.63) & & $(11.51)$ & $(14.80)$ \\
\hline \multirow[t]{2}{*}{$\phi$} & & 0.567 & 0.560 & 0.607 & $0 .^{\dagger}$ \\
\hline & & $(18.27)$ & $(11.72)$ & $(15.05)$ & \\
\hline \multirow[t]{2}{*}{$\sigma_{\varepsilon}^{2}$} & & 0.020 & 0.020 & $0.024^{\dagger}$ & 0.003 \\
\hline & & (10.37) & $(7.53)$ & & $(9.77)$ \\
\hline \multirow[t]{2}{*}{$\gamma_{00}$} & & 0.111 & & 0.100 & \\
\hline & & (14.35) & & (16.13) & \\
\hline
\end{tabular}

Data are log earnings residuals from a regression on age,

education and year dummies. $\gamma_{00}$ is the sample variance of $y_{0}$.

$t$-ratios robust to conditional heteroskedasticity.

$m 1$ and $m 2$ are serial correlation tests for differenced errors.

$\left(\phi, \sigma_{\varepsilon}^{2}\right)$ are regression coeffs. of $\left(\bar{y}-\alpha \bar{y}_{-1}\right)$ on $y_{0} .{ }^{\dagger}$ Implied by constraint. 
Table 2 (continued)

Autoregressive Model of Earnings

AR(2) Estimates for Spanish Data, 1994-1999

$N=632, T^{0}=6$

\begin{tabular}{|c|c|c|c|c|c|}
\hline & WG & GMM1 & GMM2 & System-GMM & \\
\hline \multirow[t]{2}{*}{$\alpha_{1}$} & -0.131 & 0.112 & 0.138 & 0.311 & \\
\hline & $(5.06)$ & $(1.20)$ & $(1.58)$ & $(7.91)$ & \\
\hline \multirow[t]{2}{*}{$\alpha_{2}$} & -0.118 & 0.051 & 0.070 & 0.176 & \\
\hline & $(3.78)$ & $(0.93)$ & $(1.41)$ & $(4.87)$ & \\
\hline Sargan test (df) & & & $4.21(7)$ & $16.02(11)$ & \\
\hline$m 1$ & & -6.41 & -7.02 & -11.56 & \\
\hline \multirow[t]{4}{*}{$m 2$} & & -0.75 & -0.87 & -1.55 & \\
\hline & & lihood-ba & ed Estimat & & \\
\hline & BCS & $\mathrm{RML}(\mathrm{r})$ & RML(nr) & RML(r) & $\operatorname{RML}(\mathrm{r})$ \\
\hline & (robust) & (robust) & (homosk.) & (mean stat.) & $(\phi=0)$ \\
\hline \multirow[t]{2}{*}{$\alpha_{1}$} & 0.218 & 0.201 & 0.210 & 0.300 & 0.600 \\
\hline & $(4.47)$ & $(4.89)$ & $(2.73)$ & $(4.69)$ & $(25.40)$ \\
\hline \multirow[t]{2}{*}{$\alpha_{2}$} & 0.104 & 0.094 & 0.100 & 0.102 & 0.338 \\
\hline & $(2.57)$ & $(2.47)$ & $(1.35)$ & $(2.16)$ & $(15.90)$ \\
\hline \multirow[t]{2}{*}{$\sigma_{1}^{2}(1996)$} & 0.022 & 0.022 & 0.023 & 0.026 & 0.037 \\
\hline & $(7.93)$ & $(8.69)$ & $(25.14)$ & $(7.17)$ & (11.90) \\
\hline \multirow[t]{2}{*}{$\sigma_{2}^{2}(1997)$} & 0.025 & 0.024 & & 0.026 & 0.035 \\
\hline & $(7.34)$ & $(9.15)$ & & $(8.84)$ & $(13.59)$ \\
\hline \multirow[t]{2}{*}{$\sigma_{3}^{2}(1998)$} & 0.023 & 0.023 & & 0.024 & 0.033 \\
\hline & $(8.85)$ & $(9.87)$ & & $(10.10)$ & $(12.85)$ \\
\hline \multirow[t]{2}{*}{$\sigma_{4}^{2}(1999)$} & 0.024 & 0.024 & & 0.034 & 0.035 \\
\hline & $(10.68)$ & (11.34) & & (6.13) & (13.04) \\
\hline \multirow[t]{2}{*}{$\phi_{1}$} & & 0.253 & 0.247 & & 0. \\
\hline & & $(5.39)$ & $(5.50)$ & & \\
\hline \multirow[t]{2}{*}{$\phi_{2}$} & & 0.334 & 0.326 & & 0. \\
\hline & & $(6.51)$ & (6.12) & & \\
\hline \multirow[t]{2}{*}{$\sigma_{\varepsilon}^{2}$} & & 0.016 & 0.015 & & 0.005 \\
\hline & & $(8.24)$ & $(7.57)$ & & (12.62) \\
\hline Root $_{1}$ & 0.450 & 0.424 & 0.437 & 0.503 & 0.954 \\
\hline Root $_{2}$ & -0.232 & -0.223 & -0.228 & -0.203 & -0.354 \\
\hline
\end{tabular}


Table 3

Autoregressive Model of Earnings

AR(1) Estimates for PSID Data, 1977-1983

\begin{tabular}{|c|c|c|c|c|c|}
\hline \multicolumn{6}{|c|}{$N=792, T^{0}=7$} \\
\hline & WG & GMM1 & GMM2 & System-GMM & \\
\hline \multirow[t]{2}{*}{$\alpha$} & 0.184 & 0.171 & 0.157 & 0.311 & \\
\hline & $(6.08)$ & $(3.37)$ & $(3.54)$ & $(9.76)$ & \\
\hline Sargan test (df) & & & $15.61(14)$ & $46.59(19)$ & \\
\hline$m 1$ & & -6.36 & -6.40 & -7.42 & \\
\hline \multirow[t]{4}{*}{$m 2$} & & 1.82 & 1.64 & 2.36 & \\
\hline & \multicolumn{4}{|c|}{ Likelihood-based Estimates } & \\
\hline & BCS & $\mathrm{RML}(\mathrm{r})$ & $\mathrm{RML}(\mathrm{nr})$ & RML(r) & $\operatorname{RML}(\mathrm{r})$ \\
\hline & (robust) & (robust) & (homosk.) & (mean stat.) & $(\phi=0)$ \\
\hline \multirow[t]{2}{*}{$\alpha$} & 0.387 & 0.367 & 0.416 & 0.366 & 0.902 \\
\hline & $(9.64)$ & $(10.09)$ & $(8.27)$ & $(10.04)$ & $(43.93)$ \\
\hline \multirow[t]{2}{*}{$\sigma_{1}^{2}(1978)$} & 0.061 & 0.059 & 0.068 & 0.059 & 0.113 \\
\hline & $(7.73)$ & $(7.83)$ & $(28.14)$ & $(7.83)$ & $(10.14)$ \\
\hline \multirow[t]{2}{*}{$\sigma_{2}^{2}(1979)$} & 0.062 & 0.058 & & 0.058 & 0.085 \\
\hline & $(6.10)$ & $(6.08)$ & & $(6.07)$ & $(8.73)$ \\
\hline \multirow[t]{2}{*}{$\sigma_{3}^{2}(1980)$} & 0.054 & 0.052 & & 0.052 & 0.079 \\
\hline & $(7.21)$ & $(7.55)$ & & $(7.54)$ & $(9.02)$ \\
\hline \multirow[t]{2}{*}{$\sigma_{4}^{2}(1981)$} & 0.046 & 0.046 & & 0.046 & 0.080 \\
\hline & $(6.62)$ & $(7.41)$ & & $(7.40)$ & $(8.79)$ \\
\hline \multirow{2}{*}{$\sigma_{5}^{2}(1982)$} & 0.094 & 0.096 & & 0.096 & 0.114 \\
\hline & $(3.55)$ & $(3.68)$ & & $(3.67)$ & $(4.66)$ \\
\hline \multirow[t]{2}{*}{$\sigma_{6}^{2}(1983)$} & 0.086 & 0.091 & & 0.091 & 0.132 \\
\hline & $(5.34)$ & $(5.31)$ & & $(5.31)$ & $(6.97)$ \\
\hline \multirow[t]{2}{*}{$\phi$} & & 0.385 & 0.352 & 0.384 & $0 .^{\dagger}$ \\
\hline & & $(11.84)$ & $(8.35)$ & $(11.75)$ & \\
\hline \multirow[t]{2}{*}{$\sigma_{\varepsilon}^{2}$} & & 0.045 & 0.042 & $0.046^{\dagger}$ & 0.008 \\
\hline & & $(9.35)$ & $(7.55)$ & & $(6.43)$ \\
\hline \multirow[t]{2}{*}{$\gamma_{00}$} & & 0.239 & & 0.237 & \\
\hline & & $(12.92)$ & & (13.34) & \\
\hline
\end{tabular}

Data are log earnings residuals from a regression on age, education and year dummies. $\gamma_{00}$ is the sample variance of $y_{0}$. * See notes to Table $1 .{ }^{\dagger}$ Value implied by constraint. 
Table 3 (continued)

Autoregressive Model of Earnings

AR(2) Estimates for PSID Data, 1977-1983

\begin{tabular}{|c|c|c|c|c|c|}
\hline \multicolumn{6}{|c|}{$N=792, T^{0}=7$} \\
\hline & WG & GMM1 & GMM2 & System-GMM & \\
\hline \multirow[t]{2}{*}{$\alpha_{1}$} & 0.135 & 0.227 & 0.250 & 0.433 & \\
\hline & $(3.61)$ & $(2.75)$ & $(3.37)$ & $(11.03)$ & \\
\hline \multirow[t]{2}{*}{$\alpha_{2}$} & -0.028 & 0.047 & 0.062 & 0.119 & \\
\hline & $(0.90)$ & $(1.17)$ & $(1.81)$ & $(3.93)$ & \\
\hline Sargan test (df) & & & $12.29(12)$ & $30.96(17)$ & \\
\hline$m 1$ & & -4.94 & -5.47 & -7.05 & \\
\hline \multirow[t]{3}{*}{$m 2$} & & 2.19 & 1.79 & 1.45 & \\
\hline & \multicolumn{4}{|c|}{ Likelihood-based Estimates } & \\
\hline & $\begin{array}{c}\text { BCS } \\
\text { (robust) }\end{array}$ & $\begin{array}{l}\mathrm{RML}(\mathrm{r}) \\
\text { (robust) }\end{array}$ & $\begin{array}{l}\text { RML(nr) } \\
\text { (homosk.) }\end{array}$ & $\begin{array}{c}\mathrm{RML}(\mathrm{r}) \\
\text { (mean stat.) }\end{array}$ & $\begin{array}{l}\mathrm{RML}(\mathrm{r}) \\
(\phi=0)\end{array}$ \\
\hline$\alpha_{1}$ & $\begin{array}{l}0.473 \\
(5.29)\end{array}$ & $\begin{array}{l}0.419 \\
(8.32)\end{array}$ & $\begin{array}{l}0.496 \\
(5.49)\end{array}$ & $\begin{array}{l}0.518 \\
(8.79)\end{array}$ & $\begin{array}{c}0.673 \\
(18.30)\end{array}$ \\
\hline$\alpha_{2}$ & $\begin{array}{l}0.157 \\
(2.78)\end{array}$ & $\begin{array}{l}0.115 \\
(3.14)\end{array}$ & $\begin{array}{l}0.176 \\
(2.55)\end{array}$ & $\begin{array}{l}0.159 \\
(3.56)\end{array}$ & $\begin{array}{l}0.260 \\
(8.26)\end{array}$ \\
\hline$\sigma_{1}^{2}(1979)$ & $\begin{array}{l}0.070 \\
(4.84)\end{array}$ & $\begin{array}{l}0.064 \\
(6.19)\end{array}$ & $\begin{array}{c}0.076 \\
(28.14)\end{array}$ & $\begin{array}{l}0.071 \\
(7.00)\end{array}$ & $\begin{array}{l}0.082 \\
(8.52)\end{array}$ \\
\hline$\sigma_{2}^{2}(1980)$ & $\begin{array}{l}0.061 \\
(5.50)\end{array}$ & $\begin{array}{l}0.056 \\
(7.48)\end{array}$ & & $\begin{array}{l}0.063 \\
(8.34)\end{array}$ & $\begin{array}{l}0.074 \\
(9.32)\end{array}$ \\
\hline$\sigma_{3}^{2}(1981)$ & $\begin{array}{l}0.057 \\
(5.21)\end{array}$ & $\begin{array}{l}0.051 \\
(7.01)\end{array}$ & & $\begin{array}{l}0.059 \\
(7.88)\end{array}$ & $\begin{array}{l}0.072 \\
(8.36)\end{array}$ \\
\hline$\sigma_{4}^{2}(1982)$ & $\begin{array}{l}0.092 \\
(3.69)\end{array}$ & $\begin{array}{l}0.097 \\
(3.71)\end{array}$ & & $\begin{array}{l}0.102 \\
(3.91)\end{array}$ & $\begin{array}{l}0.109 \\
(4.23)\end{array}$ \\
\hline$\sigma_{5}^{2}(1983)$ & $\begin{array}{l}0.091 \\
(4.88)\end{array}$ & $\begin{array}{l}0.090 \\
(5.28)\end{array}$ & & $\begin{array}{l}0.096 \\
(5.68)\end{array}$ & $\begin{array}{l}0.108 \\
(6.47)\end{array}$ \\
\hline$\phi_{1}$ & & $\begin{array}{l}0.096 \\
(2.95)\end{array}$ & $\begin{array}{l}0.065 \\
(2.06)\end{array}$ & & 0. \\
\hline$\phi_{2}$ & & $\begin{array}{l}0.262 \\
(4.73)\end{array}$ & $\begin{array}{l}0.174 \\
(3.23)\end{array}$ & & 0 . \\
\hline$\sigma_{\varepsilon}^{2}$ & & $\begin{array}{l}0.028 \\
(7.23)\end{array}$ & $\begin{array}{l}0.023 \\
(5.65)\end{array}$ & & $\begin{array}{l}0.012 \\
(8.38)\end{array}$ \\
\hline Root $_{1}$ & 0.698 & 0.607 & 0.736 & 0.735 & 0.947 \\
\hline $\operatorname{Root}_{2}$ & -0.225 & -0.189 & -0.240 & -0.217 & -0.274 \\
\hline
\end{tabular}


Table 4

ARMA Models of Earnings

RML Estimates for PSID Data, 1977-1983

\begin{tabular}{cccc}
\multicolumn{4}{c}{$N=792, T^{0}=7$} \\
\hline & ARMA $(1,1)$ & ARMA $(1,2)$ & ARMA $(2,1)$ \\
\hline$\alpha_{1}$ & 0.655 & 0.336 & 0.210 \\
& $(3.34)$ & $(1.74)$ & $(0.32)$ \\
$\alpha_{2}$ & & & 0.194 \\
& & & $(0.24)$ \\
$\psi_{1}$ & 0.205 & -0.068 & -0.175 \\
& $(1.69)$ & $(0.41)$ & $(0.18)$ \\
$\psi_{2}$ & & -0.139 & \\
& & $(2.62)$ & \\
$\sigma_{1978}^{2}$ & 0.069 & 0.057 & \\
& $(4.74)$ & $(6.63)$ & \\
$\sigma_{1979}^{2}$ & 0.063 & 0.062 & 0.065 \\
& $(6.69)$ & $(6.11)$ & $(0.48)$ \\
$\sigma_{1980}^{2}$ & 0.057 & 0.056 & 0.056 \\
& $(6.50)$ & $(7.85)$ & $(1.43)$ \\
$\sigma_{1981}^{2}$ & 0.055 & 0.049 & 0.048 \\
& $(4.80)$ & $(6.43)$ & $(1.52)$ \\
$\sigma_{1982}^{2}$ & 0.094 & 0.099 & 0.096 \\
& $(3.63)$ & $(3.68)$ & $(2.87)$ \\
$\sigma_{1983}^{2}$ & 0.093 & 0.092 & 0.089 \\
$\sigma_{\eta}^{2}$ & $(5.32)$ & $(5.36)$ & $(4.68)$ \\
& 0.021 & 0.073 & 0.064 \\
& $(0.90)$ & $(1.75)$ & $(1.20)$ \\
\hline & & &
\end{tabular}


Table 5

Simulations for the First-Order Autoregressive Model

Means and standard deviations of the estimators

\begin{tabular}{|c|c|c|c|c|c|}
\hline \multicolumn{6}{|c|}{$N=792, T^{0}=7$} \\
\hline & WG & GMM & RML(nr) & $\mathrm{RML}(\mathrm{r})$ & $\mathrm{BCS}$ \\
\hline \multicolumn{6}{|c|}{ True values: $\alpha=0.4, \bar{\sigma}_{0}^{2}=0.11$} \\
\hline$\alpha$ & $\begin{array}{l}0.178 \\
(0.015)\end{array}$ & $\begin{array}{l}0.396 \\
(0.035)\end{array}$ & $\begin{array}{l}0.430 \\
(0.021)\end{array}$ & $\begin{array}{l}0.400 \\
(0.020)\end{array}$ & $\begin{array}{l}0.400 \\
(0021)\end{array}$ \\
\hline$\sigma_{1}^{2}$ & & & & $\begin{array}{l}0.059 \\
(0.003)\end{array}$ & $\begin{array}{l}0.059 \\
(0,004)\end{array}$ \\
\hline$\sigma_{2}^{2}$ & & & & $\begin{array}{l}0.058 \\
(0.003)\end{array}$ & $\begin{array}{l}0.058 \\
(0.004)\end{array}$ \\
\hline$\sigma_{3}^{2}$ & & & & $\begin{array}{l}0.052 \\
(0.003)\end{array}$ & $\begin{array}{l}0.052 \\
(0.003)\end{array}$ \\
\hline$\sigma_{4}^{2}$ & & & & $\begin{array}{l}0.046 \\
(0.003)\end{array}$ & $\begin{array}{l}0.046 \\
(0.003)\end{array}$ \\
\hline$\sigma_{5}^{2}$ & & & & $\begin{array}{l}0.096 \\
(0.005)\end{array}$ & $\begin{array}{l}0.096 \\
(0.006)\end{array}$ \\
\hline$\sigma_{6}^{2}$ & & & & $\begin{array}{l}0.091 \\
(0.005)\end{array}$ & $\begin{array}{l}0.091 \\
(0.005)\end{array}$ \\
\hline
\end{tabular}

True values: $\alpha=0.8, \bar{\sigma}_{0}^{2}=0.28$

\begin{tabular}{llllll}
$\alpha$ & 0.488 & 0.772 & 0.882 & 0.804 & 0.804 \\
& $(0.016)$ & $(0.076)$ & $(0.028)$ & $(0.037)$ & $(0.040)$ \\
$\sigma_{1}^{2}$ & & & 0.059 & 0.059 \\
& & $(0.004)$ & $(0.004)$ \\
$\sigma_{2}^{2}$ & & 0.058 & 0.058 \\
& & $(0.004)$ & $(0.004)$ \\
$\sigma_{3}^{2}$ & & 0.052 & 0.052 \\
& & $(0.004)$ & $(0.004)$ \\
$\sigma_{4}^{2}$ & & 0.046 & 0.046 \\
& & & $(0.003)$ & $(0.003)$ \\
$\sigma_{5}^{2}$ & & 0.096 & 0.096 \\
& & & $(0.005)$ & $(0.006)$ \\
$\sigma_{6}^{2}$ & & 0.091 & 0.091 \\
& & & $(0.005)$ & $(0.005)$ \\
\hline
\end{tabular}

1000 replications. Variance values: $\sigma_{1}^{2}=0.059, \sigma_{2}^{2}=0.058$, $\sigma_{3}^{2}=0.052, \sigma_{4}^{2}=0.046, \sigma_{5}^{2}=0.096, \sigma_{6}^{2}=0.091, \sigma_{\eta}^{2}=0.07$. 
Figure 1

Relative Inefficiency Ratio (lamda $=0$ )

Homoskedastic Estimators

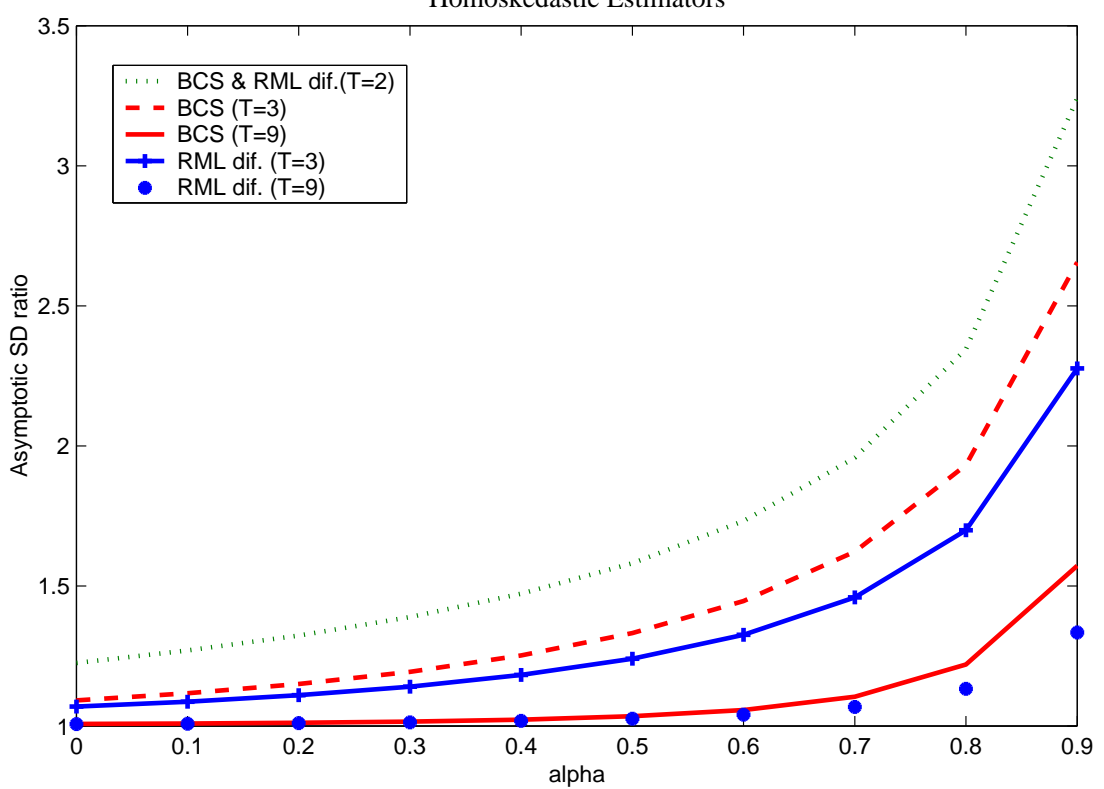

Figure 2

Relative Inefficiency Ratio (lamda $=1$ )

Homoskedastic Estimators

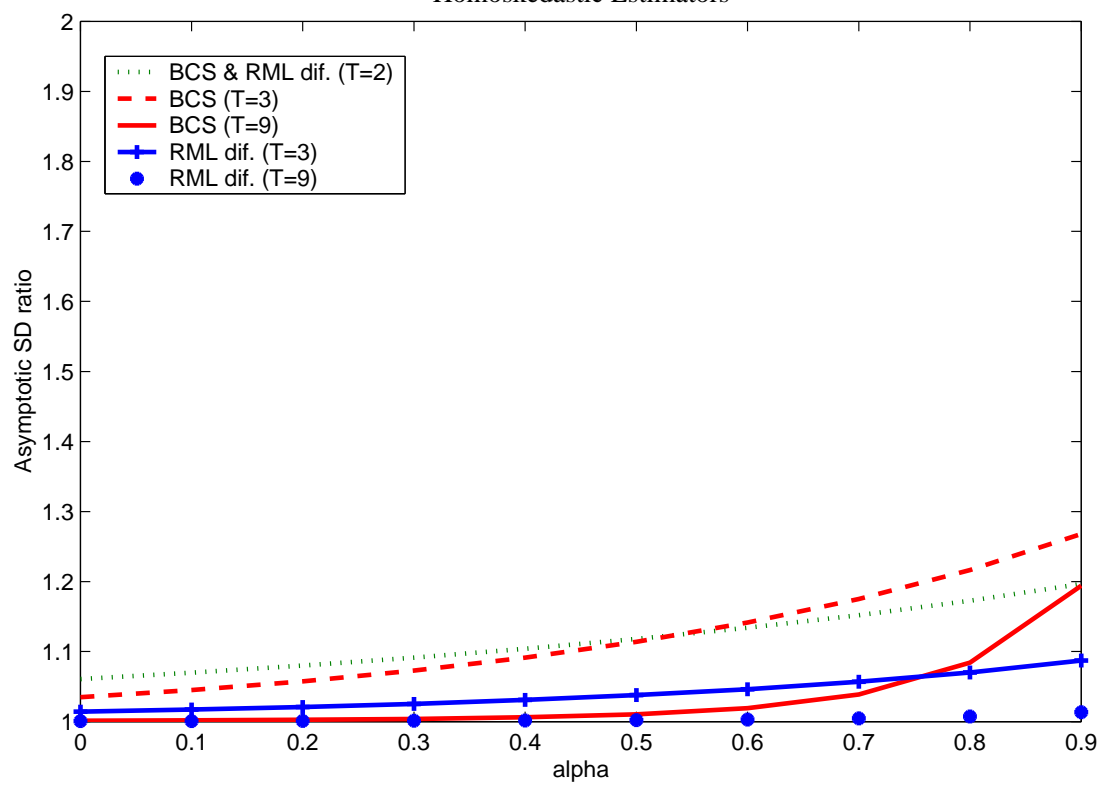


Figure 3

Relative Inefficiency Under Nonstationary Initial Variance $(\mathrm{T}=3$, alpha $=0.9$, lamda $=0$ ) Homoskedastic Estimators

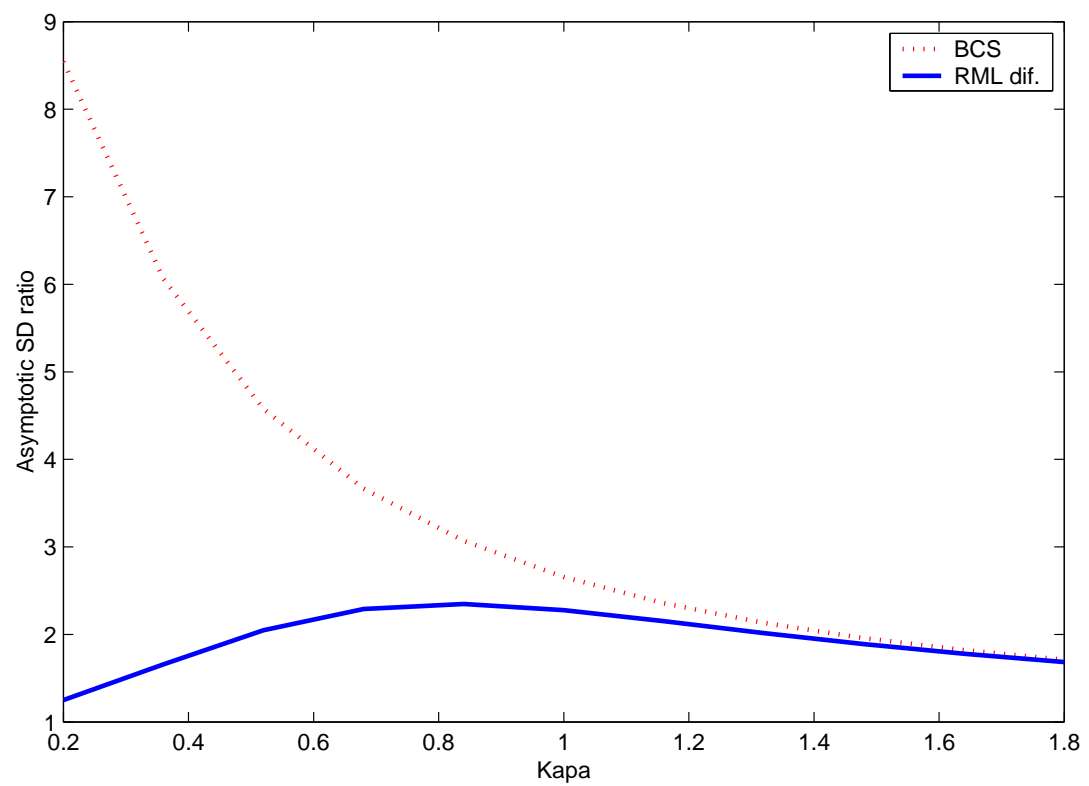

Figure 4

Relative Inefficiency Ratio (lamda=0)

Heteroskedastic Estimators

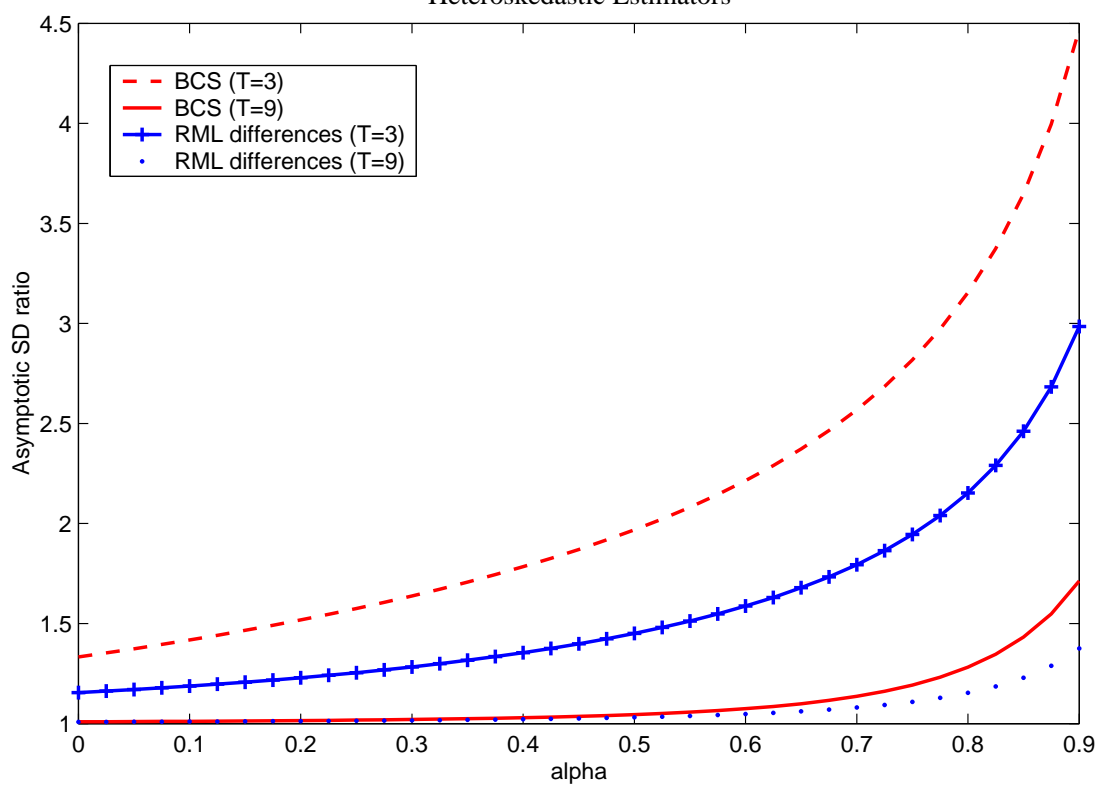


Figure 5

Relative Inefficiency Ratio (lamda=1)

Heteroskedastic Estimators

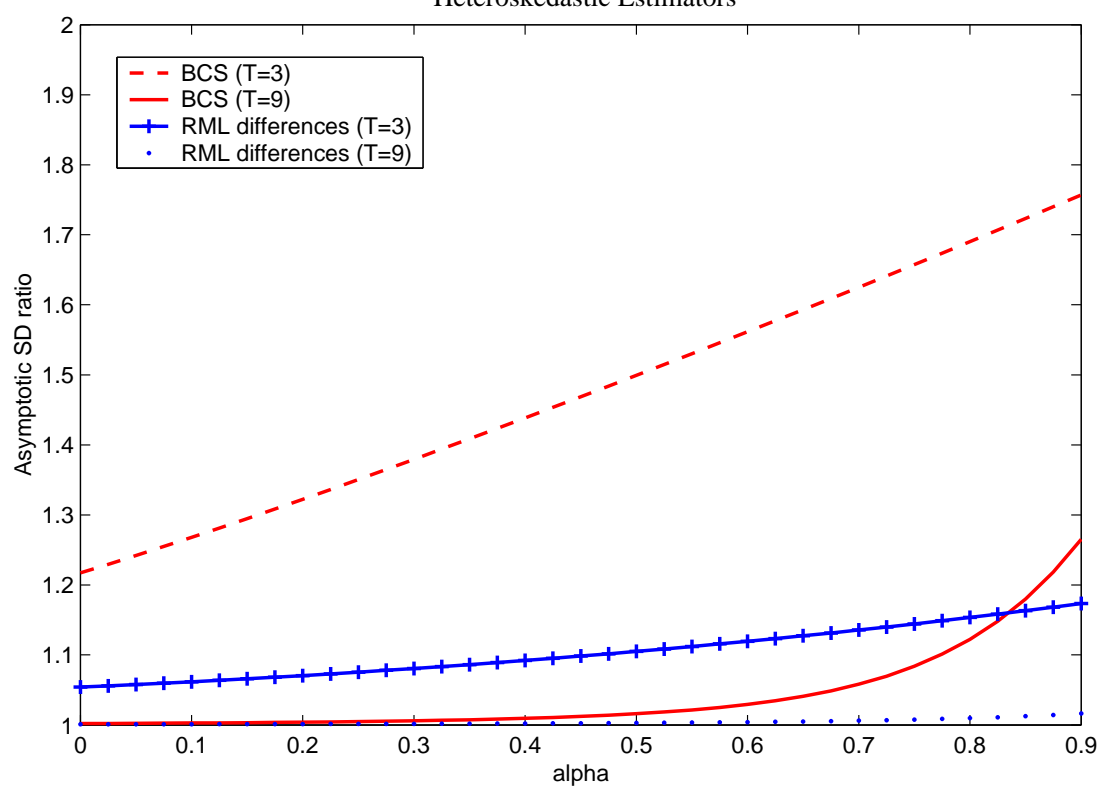

Figure 6

Asymptotic Standard Deviation Under Unit Root $(\mathrm{T}=6$, alpha $=1$, lamda $=0)$ By Location and Change in Single Break in Variances

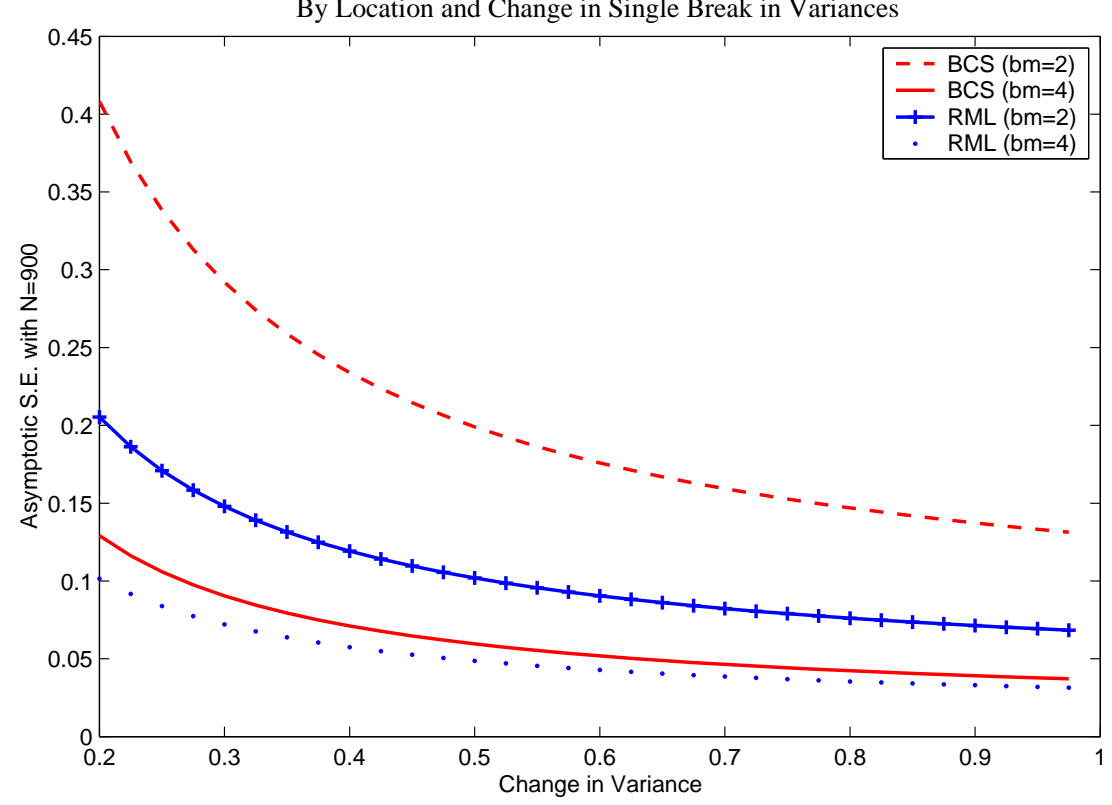


Figure 7

Distribution of Residuals in First Differences

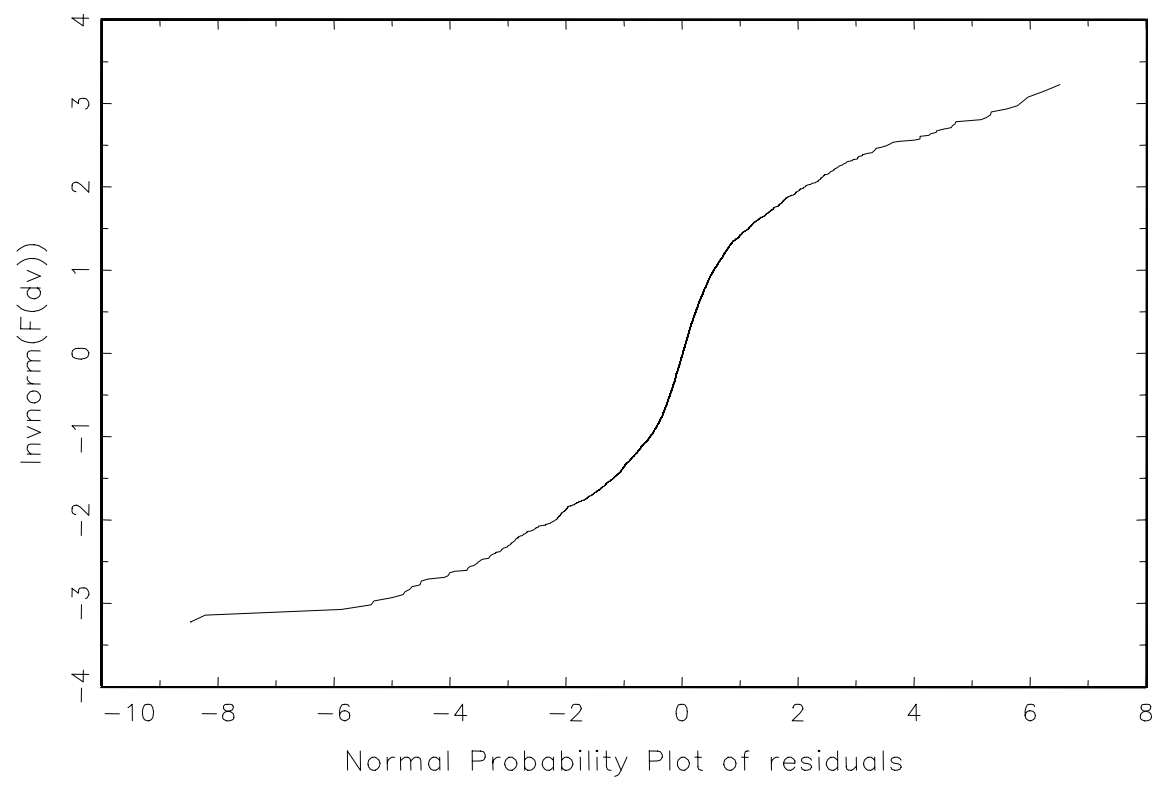

Figure 8

Graphical Test of Normality of Individual Effects

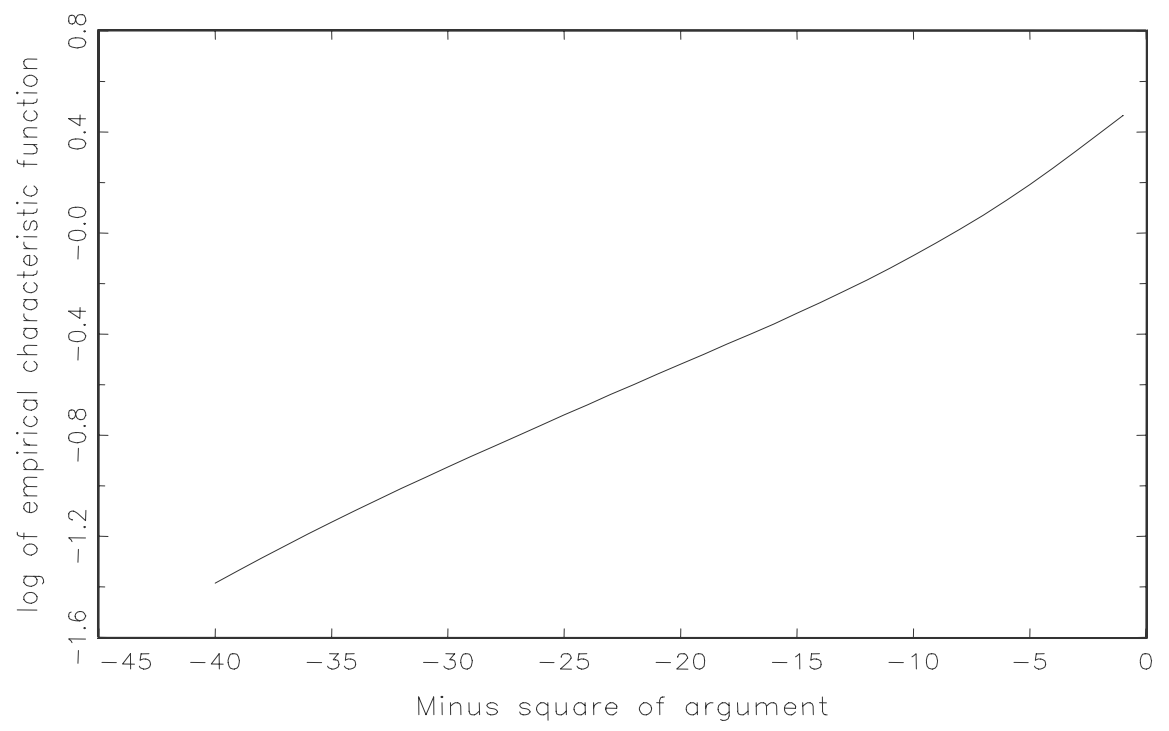


Appendix for Robust Likelihood Estimation of Dynamic Panel

Data Models by Javier Alvarez and Manuel Arellano

\section{A. Conditional Maximum Likelihood and Expected Scores}

\section{A.1. First-Order Conditions and Related Results}

Equations (3.8), (3.11): Note that $\bar{v}=v^{\prime} \Phi \iota$ and $\omega_{T}=\operatorname{Var}(\bar{v})=\left(\iota^{\prime} \Lambda^{-1} \iota\right)^{-1}$, so that $\Lambda^{-1}=\left(1 / \omega_{T}\right) \Phi$. Moreover, the equivalences in (3.7) also imply

$$
\ln \operatorname{det} \Lambda=\ln \operatorname{det}\left(D \Lambda D^{\prime}\right)+\ln \omega_{T} .
$$

Clearly $0 \leq \varphi_{t} \leq 1, \sum_{t=1}^{T} \varphi_{t}=1$, and under homoskedasticity $\varphi_{t}=1 / T$ for all $t$.

Regarding period-specific variances, taking into account that:

$$
E\left[\left(v_{t}-\bar{v}\right)^{2}\right]=\sigma_{t}^{2}+\omega_{T}-2 E\left(v_{t} \bar{v}\right)=\sigma_{t}^{2}+\omega_{T}-2 \varphi_{t} \sigma_{t}^{2}=\sigma_{t}^{2}+\omega_{T}-2 \omega_{T},
$$

we obtain expression (3.8), and also

$$
\sigma_{t}^{2}-\sigma_{t-1}^{2}=E\left[\left(v_{t}-\bar{v}\right)^{2}\right]-E\left[\left(v_{t-1}-\bar{v}\right)^{2}\right] \quad(t=2, \ldots T) .
$$

Finally, equation (3.11) is easily verified from (3.8).

Idempotent Matrices: Letting $Q=\Phi-\Phi \iota \iota^{\prime} \Phi$, note that the matrix $Q^{\dagger}=$ $I-\Phi^{1 / 2} \iota \iota^{\prime} \Phi^{1 / 2}$ is idempotent, and that $Q=\Phi^{1 / 2} Q^{\dagger} \Phi^{1 / 2}$. Also

$$
Q^{\dagger}=\Lambda^{1 / 2} D^{\prime}\left(D \Lambda D^{\prime}\right)^{-1} D \Lambda^{1 / 2}=I-\omega_{T} \Lambda^{-1 / 2} \iota \iota^{\prime} \Lambda^{-1 / 2}
$$

and $D^{\prime}\left(D \Lambda D^{\prime}\right)^{-1} D=\Lambda^{-1 / 2} Q^{\dagger} \Lambda^{-1 / 2}$. So that

$$
D^{\prime}\left(D \Lambda D^{\prime}\right)^{-1} D=\Lambda^{-1}-\omega_{T} \Lambda^{-1} \iota \iota^{\prime} \Lambda^{-1}=\omega_{T}^{-1} Q .
$$

Derivatives: Letting $\varphi=\left(\varphi_{1}, \ldots, \varphi_{T}\right)^{\prime}=\Phi \iota$, we have the following result:

$$
\frac{\partial \varphi}{\partial \theta^{\prime}}=-\left(\Phi-\Phi \iota \iota^{\prime} \Phi\right) \Lambda^{-1}=-D^{\prime}\left(D \Lambda D^{\prime}\right)^{-1} D \Phi
$$

To see this recall that $\varphi_{s}=\omega_{T} / \sigma_{T}^{2}$ and consider

$$
d \varphi=\omega_{T} \frac{\partial}{\partial \theta^{\prime}}\left(\begin{array}{c}
1 / \sigma_{1}^{2} \\
\vdots \\
1 / \sigma_{T}^{2}
\end{array}\right) d \theta+\left(\begin{array}{c}
1 / \sigma_{1}^{2} \\
\vdots \\
1 / \sigma_{T}^{2}
\end{array}\right) \frac{\partial \omega_{T}}{\partial \theta^{\prime}} d \theta
$$

Also using

$$
\frac{\partial \omega_{T}}{\partial \sigma_{s}^{2}}=\frac{1 / \sigma_{s}^{4}}{\left(\sigma_{1}^{-2}+\ldots+\sigma_{T}^{-2}\right)^{2}}=\varphi_{s}^{2}
$$


we get

$$
\begin{aligned}
\frac{\partial \varphi}{\partial \theta^{\prime}} & =-\omega_{T}\left(\begin{array}{ccc}
1 / \sigma_{1}^{4} & \ldots & 0 \\
\vdots & \ddots & \vdots \\
0 & \ldots & 1 / \sigma_{T}^{4}
\end{array}\right)+\left(\begin{array}{c}
1 / \sigma_{1}^{2} \\
\vdots \\
1 / \sigma_{T}^{2}
\end{array}\right)\left(\begin{array}{ccc}
\varphi_{1}^{2} & \ldots & \varphi_{T}^{2}
\end{array}\right) \\
& =-\frac{1}{\omega_{T}} \Phi \Phi-\frac{1}{\omega_{T}}\left(\begin{array}{c}
\varphi_{1} \\
\vdots \\
\varphi_{T}
\end{array}\right)\left(\begin{array}{lll}
\varphi_{1} & \ldots & \varphi_{T}
\end{array}\right) \Phi=-\frac{1}{\omega_{T}}\left(\Phi-\Phi \iota \iota^{\prime} \Phi\right) \Phi .
\end{aligned}
$$

First-Order Conditions Formulae (3.21)-(3.22): For a matrix $A=\left(a_{1}, \ldots, a_{n}\right)^{\prime}$, we use the notation $\operatorname{vec}(A)=\left(a_{1}^{\prime}, \ldots, a_{n}^{\prime}\right)^{\prime}$ and $A \otimes B=\left\{a_{j k} B\right\}$. The derivative of $L_{C}$ with respect $\omega_{T}$ is

$$
\frac{\partial L_{C}}{\partial \omega_{T}}=\frac{1}{\omega_{T}^{2}} \sum_{i=1}^{N}\left[v_{i}^{\prime}\left(\Phi-\Phi \iota \iota^{\prime} \Phi\right) v_{i}-(T-1) \omega_{T}\right] .
$$

The concentrated likelihood with respect to $\omega_{T}$ is

$$
L_{C}^{*}=\frac{N}{2} \sum_{t=1}^{T} \ln \varphi_{t}-\frac{N(T-1)}{2} \ln \sum_{i=1}^{N} \sum_{t=1}^{T} \varphi_{t}\left(v_{i t}-\bar{v}_{i}\right)^{2},
$$

and the Lagrangean

$$
\mathcal{L}=L_{C}^{*}+\lambda\left(1-\sum_{t=1}^{T} \varphi_{t}\right)
$$

so that

$$
\begin{aligned}
\frac{\partial \mathcal{L}}{\partial \varphi_{t}} & =\frac{N}{2} \frac{1}{\varphi_{t}}-\frac{1}{2 \widehat{\omega}_{T}} \sum_{i=1}^{N}\left[\left(v_{i t}-\bar{v}_{i}\right)^{2}-2 v_{i t} \bar{v}_{i}\left(1-\sum_{s=1}^{T} \varphi_{s}\right)\right]-\lambda \\
\frac{\partial \mathcal{L}}{\partial \lambda} & =1-\sum_{t=1}^{T} \varphi_{t} .
\end{aligned}
$$

Inserting the restriction, the first-order conditions for the weights are

$$
\frac{1}{\varphi_{t}}=\frac{1}{\widehat{\omega}_{T}} \frac{1}{N} \sum_{i=1}^{N}\left(v_{i t}-\bar{v}_{i}\right)^{2}+\lambda,
$$

and taking first-differences to eliminate the Lagrange multiplier

$$
\frac{\widehat{\omega}_{T}}{\varphi_{t}}-\frac{\widehat{\omega}_{T}}{\varphi_{t-1}}=\frac{1}{N} \sum_{i=1}^{N}\left[\left(v_{i t}-\bar{v}_{i}\right)^{2}-\left(v_{i(t-1)}-\bar{v}_{i}\right)^{2}\right] .
$$

Nonnegativity constraints: The nonnegativity constraints $\sigma_{t}^{2}>0$ may be enforced through the parameterization $\left(\omega_{T}, \varphi_{1}, \ldots, \varphi_{T}\right)$ imposing adding-up and non-negativity restrictions to the weights. Alternatively, transformed variances for errors in orthogonal deviations can be used, which confine nonnegativity restrictions to $\sigma_{T}^{2}$. This transformation is discussed next. 


\section{A.2. Heteroskedastic Orthogonal Deviations}

The following equivalences also hold

$$
\begin{gathered}
v^{\prime} D^{\prime}\left(D \Lambda D^{\prime}\right)^{-1} D v=\sum_{t=1}^{T-1} \frac{\widetilde{v}_{t}^{2}}{\widetilde{\sigma}_{t}^{2}} \\
\ln \operatorname{det}\left(D \Lambda D^{\prime}\right)=\sum_{t=1}^{T} \ln \sigma_{t}^{2}+\ln \left(\sigma_{1}^{-2}+\ldots+\sigma_{T}^{-2}\right)=\sum_{t=1}^{T-1} \ln \widetilde{\sigma}_{t}^{2}
\end{gathered}
$$

where the heteroskedastic orthogonal deviations are given by

$$
\begin{aligned}
& \widetilde{v}_{t}= \begin{cases}v_{T-1}-v_{T} & \text { for } t=T-1 \\
v_{t}-\frac{\sigma_{t+1}^{-2} v_{t+1}+\ldots+\sigma_{T}^{-2} v_{T}}{\sigma_{t+1}^{-2}+\ldots+\sigma_{T}^{-2}} & \text { for } t=T-2, \ldots, 1\end{cases} \\
& \tilde{\sigma}_{t}^{2}= \begin{cases}\sigma_{T-1}^{2}+\sigma_{T}^{2} & \text { for } t=T-1 \\
\sigma_{t}^{2}+\frac{1}{\sigma_{t+1}^{-2}+\ldots+\sigma_{T}^{-2}} & \text { for } t=T-2, \ldots, 1\end{cases}
\end{aligned}
$$

or

$$
\widetilde{v}_{t}= \begin{cases}v_{T-1}-v_{T} & \text { for } t=T-1 \\ \left(v_{t}-v_{t+1}\right)+\lambda_{t+1} \widetilde{v}_{t+1} & \text { for } t=T-2, \ldots, 1\end{cases}
$$

where $\lambda_{t}=\sigma_{t}^{2} / \widetilde{\sigma}_{t}^{2},(t=T-1, \ldots, 1)$.

To clarify the mapping between $\left(\sigma_{1}^{2}, \ldots, \sigma_{T}^{2}\right)$ and $\left(\widetilde{\sigma}_{1}^{2}, \ldots, \widetilde{\sigma}_{T-1}^{2}\right)$ note that

$$
\begin{gathered}
E\left[\left(v_{T-1}-v_{T}\right)\left(v_{T-2}-v_{T}\right)\right]=\sigma_{T}^{2} \\
E\left(\widetilde{v}_{t}\right)=\widetilde{\sigma}_{t}^{2} \quad(t=T-1, \ldots, 1) .
\end{gathered}
$$

So we identify $\sigma_{T}^{2}$ as a covariance between $\left(v_{T-1}-v_{T}\right)$ and $\left(v_{T-2}-v_{T}\right)$, and $\tilde{\sigma}_{T-1}^{2}$ as the variance of $\widetilde{v}_{T-1}=\left(v_{T-1}-v_{T}\right)$, so that $\sigma_{T-1}^{2}=\widetilde{\sigma}_{T-1}^{2}-\sigma_{T}^{2}$. We can get

$$
\lambda_{T-1}=\frac{\sigma_{T-1}^{2}}{\widetilde{\sigma}_{T-1}^{2}}=\frac{\sigma_{T-1}^{2}}{\sigma_{T-1}^{2}+\sigma_{T}^{2}}
$$

and use it to form

$$
\widetilde{v}_{T-2}=\left(v_{T-2}-v_{T-1}\right)+\lambda_{T-1} \widetilde{v}_{T-1},
$$

which allows us to get $\widetilde{\sigma}_{T-2}^{2}$. Now we can get $\sigma_{T-2}^{2}=\widetilde{\sigma}_{T-2}^{2}-1 /\left(\sigma_{T-1}^{-2}+\sigma_{T}^{-2}\right)$, $\lambda_{T-2}=\sigma_{T-2}^{2} / \widetilde{\sigma}_{T-2}^{2}$, and proceed recursively to obtain the remaining terms. Note that the $\widetilde{\sigma}_{t}^{2}$ will be nonnegative by construction, so that the non-negativity problem is confined to $\sigma_{T}^{2}$. 


\section{A.3. Score Bias Function}

Proof of (3.24): We have

$$
\begin{gathered}
E\left[X_{i}^{\prime} D^{\prime}\left(D \Lambda D^{\prime}\right)^{-1} D v_{i}\right]=E\left(X_{i}^{\prime} \Lambda^{-1} v_{i}\right)-\omega_{T} E\left(X_{i}^{\prime} \Lambda^{-1} \iota \iota^{\prime} \Lambda^{-1} v_{i}\right) \\
=-\omega_{T} E\left(\begin{array}{c}
x_{1 i}^{\prime} \Lambda^{-1} \iota \iota^{\prime} \Lambda^{-1} v_{i} \\
\vdots \\
x_{p i}^{\prime} \Lambda^{-1} \iota \iota^{\prime} \Lambda^{-1} v_{i}
\end{array}\right)=-\omega_{T}\left(\begin{array}{c}
\iota^{\prime} \Lambda^{-1} E\left(x_{1 i} v_{i}^{\prime}\right) \Lambda^{-1} \iota \\
\vdots \\
\iota^{\prime} \Lambda^{-1} E\left(x_{p i} v_{i}^{\prime}\right) \Lambda^{-1} \iota
\end{array}\right)
\end{gathered}
$$

To obtain an expression for $E\left(x_{j i} v_{i}^{\prime}\right)$ we need to develop a suitable notation. Let us write

$$
\left(\begin{array}{cc}
I_{p} & 0 \\
B_{T p} & B_{T}
\end{array}\right)\left(\begin{array}{c}
y_{i}^{0} \\
y_{i}
\end{array}\right)=\left(\begin{array}{c}
y_{i}^{0} \\
\eta_{i} \iota+v_{i}
\end{array}\right)
$$

where

$$
\left(\begin{array}{ll}
B_{T p} & B_{T}
\end{array}\right)=\left(\begin{array}{ccccccccccc}
-\alpha_{p} & -\alpha_{p-1} & \ldots & -\alpha_{1} & 1 & 0 & \ldots & 0 & \ldots & 0 & 0 \\
0 & -\alpha_{p} & \ldots & -\alpha_{2} & -\alpha_{1} & 1 & & 0 & \ldots & 0 & 0 \\
0 & 0 & \ddots & & -\alpha_{2} & -\alpha_{1} & \ddots & 0 & \ldots & 0 & 0 \\
\vdots & \vdots & & & & \ddots & \ddots & \ddots & & \vdots & \vdots \\
0 & 0 & & 0 & 0 & & & 0 & & 1 & 0 \\
0 & 0 & \ldots & 0 & 0 & 0 & \ldots & -\alpha_{p} & \ldots & -\alpha_{1} & 1
\end{array}\right) .
$$

Moreover,

$$
\left(\begin{array}{c}
y_{i}^{0} \\
y_{i}
\end{array}\right)=\left(\begin{array}{cc}
I_{p} & 0 \\
\bar{C}_{T p} & \bar{C}_{T}
\end{array}\right)\left(\begin{array}{c}
y_{i}^{0} \\
\eta_{i} \iota+v_{i}
\end{array}\right)
$$

where $\bar{C}_{T}=B_{T}^{-1}$ and $\bar{C}_{T p}=-B_{T}^{-1} B_{T p}$, so that

$$
y_{i}=\bar{C}_{T p} y_{i}^{0}+\eta_{i} \bar{C}_{T} \iota+\bar{C}_{T} v_{i} .
$$

Thus,

$$
E\left(y_{i} v_{i}^{\prime}\right)=\bar{C}_{T p} E\left(y_{i}^{0} v_{i}^{\prime}\right)+\bar{C}_{T} E\left(v_{i} v_{i}^{\prime}\right)=\bar{C}_{T} \Lambda .
$$

Let us consider now an expression for $x_{j i}=\left(y_{i(1-j)}, \ldots, y_{i 0}, y_{i 1}, \ldots, y_{i(T-j)}\right)^{\prime}$. Since we have

$$
\left(\begin{array}{c}
y_{i(1-j)} \\
\vdots \\
y_{i 0}
\end{array}\right)=\left(\begin{array}{ll}
0 & I_{j}
\end{array}\right) y_{i}^{0}
$$

and

$$
\left(\begin{array}{c}
y_{i 1} \\
\vdots \\
y_{i(T-j)}
\end{array}\right)=\bar{C}_{(T-j) p} y_{i}^{0}+\eta_{i} \bar{C}_{T-j} \iota_{T-j}+\bar{C}_{T-j}\left(\begin{array}{c}
v_{i 1} \\
\vdots \\
v_{i(T-j)}
\end{array}\right)
$$


we can write $x_{j i}$ as

$x_{j i}=\left(\begin{array}{cc}0 & I_{j} \\ \bar{C}_{(T-j) p}\end{array}\right) y_{i}^{0}+\eta_{i}\left(\begin{array}{cc}0 & 0 \\ \bar{C}_{T-j} & 0\end{array}\right)\left(\begin{array}{c}\iota_{T-j} \\ \iota_{j}\end{array}\right)+\left(\begin{array}{cc}0 & 0 \\ \bar{C}_{T-j} & 0\end{array}\right)\left[\begin{array}{c}\left(\begin{array}{c}v_{i 1} \\ \vdots \\ v_{i(T-j)}\end{array}\right) \\ \left(\begin{array}{c}v_{i(T-j+1)} \\ \vdots \\ v_{i T}\end{array}\right)\end{array}\right]$

or

$$
x_{j i}=C_{T p}^{j} y_{i}^{0}+\eta_{i} C_{j} \iota+C_{j} v_{i} \quad(j=1, \ldots, p)
$$

where

$$
C_{j}=\left(\begin{array}{cc}
0 & 0 \\
\bar{C}_{T-j} & 0
\end{array}\right) \quad C_{T p}^{j}=\left(\begin{array}{cc}
0 & I_{j} \\
\bar{C}_{(T-j) p}
\end{array}\right) .
$$

Therefore,

$$
E\left(x_{j i} v_{i}^{\prime}\right)=C_{j} \Lambda
$$

and in view of the previous expression

$$
E\left[X_{i}^{\prime} D^{\prime}\left(D \Lambda D^{\prime}\right)^{-1} D v_{i}\right]=-\omega_{T}\left(\begin{array}{c}
\iota^{\prime} \Lambda^{-1} C_{1} \iota \\
\vdots \\
\iota^{\prime} \Lambda^{-1} C_{p} \iota
\end{array}\right)=-\left(\begin{array}{c}
\varphi^{\prime} C_{1} \iota \\
\vdots \\
\varphi^{\prime} C_{p} \iota
\end{array}\right)
$$

Moreover, note that weighted averages are given by

$$
\bar{x}_{j i}=\varphi^{\prime} x_{j i}=\eta_{i}\left(\varphi^{\prime} C_{j} \iota\right)+\left(\varphi^{\prime} C_{T p}^{j}\right) y_{i}^{0}+\varphi^{\prime} C_{j} v_{i} \quad(j=1, \ldots, p) .
$$

Also note that the variance of the average error can be eliminated to give rise to moment conditions that only depend on $\alpha$ and the weights.

Integral (3.38) of the Bias Function when $p=1$ :

To see that the integral of $h_{T}(\alpha, \varphi)$ when $p=1$ is given by (3.38) note that using

$$
\begin{aligned}
h_{T}(\alpha, \varphi) & =\sum_{t=1}^{T-1}\left(1+\alpha+. .+\alpha^{t-1}\right) \varphi_{t+1} \\
& =\sum_{t=1}^{T-1} \varphi_{t+1}+\alpha \sum_{t=2}^{T-1} \varphi_{t+1}+\alpha^{2} \sum_{t=3}^{T-1} \varphi_{t+1}+\ldots+\alpha^{T-2} \varphi_{T}
\end{aligned}
$$

we can write

$$
\begin{aligned}
b_{T}(\alpha, \varphi) & =\alpha \sum_{s=1}^{T-1} \varphi_{s+1}+\frac{\alpha^{2}}{2} \sum_{s=2}^{T-1} \varphi_{s+1}+\frac{\alpha^{3}}{3} \sum_{s=3}^{T-1} \varphi_{s+1}+. .+\frac{\alpha^{T-1}}{T-1} \varphi_{T} \\
& =\sum_{t=1}^{T-1} \frac{\left(\varphi_{t+1}+\ldots+\varphi_{T}\right)}{t} \alpha^{t} .
\end{aligned}
$$


Derivatives of $b_{T}(\alpha, \varphi)$ with respect to $\varphi_{t}$ are:

$$
\frac{\partial b_{T}(\alpha, \varphi)}{\partial \varphi_{t}}= \begin{cases}0 & \text { for } t=1 \\ \sum_{s=1}^{t-1} \frac{\alpha^{s}}{s} & \text { for } t>1\end{cases}
$$

and in view of (A.2):

$$
\frac{\partial b_{T}(\alpha, \varphi)}{\partial \theta}=\left(\frac{\partial \varphi}{\partial \theta^{\prime}}\right)^{\prime} \frac{\partial b_{T}(\alpha, \varphi)}{\partial \varphi}=-\Phi D^{\prime}\left(D \Lambda D^{\prime}\right)^{-1} D\left(\begin{array}{c}
0 \\
\alpha \\
\alpha+\frac{\alpha^{2}}{2} \\
\alpha+\frac{\alpha^{2}}{2}+\frac{\alpha^{3}}{3} \\
\vdots
\end{array}\right)
$$

\section{Proof of (4.8) and (4.9) for the Random Effects Scores:}

Let $\xi_{i}=\eta_{i}-\phi y_{i 0}$, so that

$$
\sigma_{\varepsilon}^{2}=\operatorname{Var}\left(\bar{v}_{i}\right)+\operatorname{Var}\left(\xi_{i}\right) .
$$

Using this expression and (A.15) we have

$$
\begin{aligned}
\frac{1}{\sigma_{\varepsilon}^{2}} E\left[\bar{x}_{i}\left(\bar{u}_{i}-\phi^{\prime} y_{i}^{0}\right)\right] & =\frac{1}{\sigma_{\varepsilon}^{2}}\left\{E\left(\bar{x}_{i} \bar{v}_{i}\right)+E\left[\bar{x}_{i}\left(\eta_{i}-\phi^{\prime} y_{i}^{0}\right)\right]\right\} \\
& =\frac{1}{\sigma_{\varepsilon}^{2}}\left[\omega_{T}^{2} \Lambda^{-1} \iota^{\prime} E\left(v_{i} X_{i}^{\prime}\right) \Lambda^{-1} \iota+h_{T}(\alpha, \varphi) E\left(\eta_{i} \xi_{i}\right)\right] \\
& =\frac{1}{\sigma_{\varepsilon}^{2}}\left[\omega_{T} h_{T}(\alpha, \varphi)+h_{T}(\alpha, \varphi) \operatorname{Cov}\left(\eta_{i}, \xi_{i}\right)\right] \\
& =h_{T}(\alpha, \varphi) \frac{1}{\sigma_{\varepsilon}^{2}}\left[\operatorname{Var}\left(\bar{v}_{i}\right)+\operatorname{Var}\left(\xi_{i}\right)\right]=h_{T}(\alpha, \varphi) .
\end{aligned}
$$

This proves result (4.8). Turning to (4.9), we have

$$
\begin{aligned}
& \quad E\left[\frac{1}{\sigma_{\varepsilon}^{2}} \Phi D^{\prime}\left(D \Lambda D^{\prime}\right)^{-1} D v_{i}\left(\bar{u}_{i}-\phi^{\prime} y_{i}^{0}\right)\right]= \\
& =\frac{1}{\sigma_{\varepsilon}^{2}} \Phi D^{\prime}\left(D \Lambda D^{\prime}\right)^{-1} E\left(D v_{i} \bar{v}_{i}\right) \\
& =\frac{1}{\sigma_{\varepsilon}^{2}} \Phi D^{\prime}\left(D \Lambda D^{\prime}\right)^{-1} D E\left(v_{i} v_{i}^{\prime}\right) \Phi \iota \\
& =\frac{1}{\sigma_{\varepsilon}^{2}} \Phi D^{\prime}\left(D \Lambda D^{\prime}\right)^{-1} D \Lambda \Phi \iota \\
& =\frac{\omega_{T}}{\sigma_{\varepsilon}^{2}} \Phi D^{\prime}\left(D \Lambda D^{\prime}\right)^{-1} D \Lambda \Lambda^{-1} \iota=\frac{\omega_{T}}{\sigma_{\varepsilon}^{2}} \Phi D^{\prime}\left(D \Lambda D^{\prime}\right)^{-1} D \iota=0 .
\end{aligned}
$$




\section{B. Asymptotic Variances of Estimators Under Normality}

This Appendix presents the formulae for the asymptotic variances of RML and BCS estimators used for the inefficiency calculations reported in the main body of the paper. They are calculated under the assumption of normality for both homoskedastic and heteroskedastic estimators when $p=1$. These formulas are not suggested for empirical standard error calculations (for which we use robust sample expressions that remain consistent under conditional heteroskedasticity and nonnormality), but in order to facilitate numerical comparisons of relative efficiency among alternative estimators.

\section{B.1. Asymptotic Variance of the RML-dif Estimator}

Letting $\eta_{i}^{\dagger}=\eta_{i}-(1-\alpha) y_{i 0}$, the $\mathrm{AR}(1)$ model can be written as

$$
\begin{aligned}
\Delta y_{i 1} & =\eta_{i}^{\dagger}+v_{i 1} \\
\Delta y_{i t} & =\alpha \Delta y_{i(t-1)}+\Delta v_{i t} \quad(t=2, \ldots, T)
\end{aligned}
$$

or in vector notation

$$
B\left(\begin{array}{c}
\Delta y_{i 1} \\
\vdots \\
\Delta y_{i T}
\end{array}\right)=D^{\dagger}\left(\begin{array}{c}
\eta_{i}^{\dagger}+v_{i 1} \\
\vdots \\
\eta_{i}^{\dagger}+v_{i T}
\end{array}\right) \equiv D^{\dagger} u_{i}^{\dagger}
$$

where $B$ and $D^{\dagger}$ are $T \times T$ matrices of the form

$$
B=\left(\begin{array}{ccccc}
1 & 0 & \ldots & 0 & 0 \\
-\alpha & 1 & \ldots & 0 & 0 \\
\vdots & & \ddots & & \vdots \\
0 & 0 & \ldots & -\alpha & 1
\end{array}\right), \quad D^{\dagger}=\left(\begin{array}{ccccc}
1 & 0 & \ldots & 0 & 0 \\
& D & &
\end{array}\right)
$$

Moreover,

$$
\operatorname{Var}\left(D^{\dagger} u_{i}^{\dagger}\right)=D^{\dagger}\left(\sigma_{\eta \dagger}^{2} \iota \iota^{\prime}+\Lambda\right) D^{\dagger \prime}
$$

where $\sigma_{\eta \dagger}^{2}=\operatorname{Var}\left(\eta_{i}^{\dagger}\right)$ and under homoskedascity $\Lambda=\sigma^{2} I_{T}$.

Therefore,

$$
\operatorname{Var}\left(\begin{array}{c}
\Delta y_{i 1} \\
\vdots \\
\Delta y_{i T}
\end{array}\right)=B^{-1} D^{\dagger}\left(\sigma_{\eta \dagger}^{2} \iota \iota^{\prime}+\Lambda\right) D^{\dagger \prime} B^{-1 \prime} \equiv \Omega(\gamma)
$$

where $\gamma=\left(\alpha, \sigma_{1}^{2}, \ldots, \sigma_{T}^{2}, \sigma_{\eta \dagger}^{2}\right)^{\prime}$. 
Moreover, note that the heteroskedastic marginal MLE for the data in differences can be written as

$\left(\widehat{\alpha}_{D}, \widehat{\sigma}_{1}^{2} \ldots, \widehat{\sigma}_{T}^{2}, \widehat{\sigma}_{\eta \dagger}^{2}\right)=\arg \min \left[\ln \operatorname{det} \Omega(\gamma)+\frac{1}{N} \sum_{i=1}^{N}\left(\Delta y_{i 1}, \ldots, \Delta y_{i T}\right) \Omega^{-1}(\gamma)\left(\begin{array}{c}\Delta y_{i 1} \\ \vdots \\ \Delta y_{i T}\end{array}\right)\right]$.

Thus, under normality the asymptotic variance matrix of $\left(\widehat{\alpha}_{D}, \widehat{\sigma}_{1}^{2} \ldots, \widehat{\sigma}_{T}^{2}, \widehat{\sigma}_{\eta \dagger}^{2}\right)$ is given by ${ }^{15}$

$$
2\left\{H(\gamma)^{\prime} \mathcal{D}^{\prime}\left[\Omega^{-1}(\gamma) \otimes \Omega^{-1}(\gamma)\right] \mathcal{D} H(\gamma)\right\}^{-1}
$$

where

$$
H(\gamma)=\frac{\partial v e c h[\Omega(\gamma)]}{\partial \gamma^{\prime}}
$$

and $\mathcal{D}$ is the selection matrix

$$
\mathcal{D}=\frac{\partial \operatorname{vec} \Omega}{\partial(\operatorname{vech} \Omega)^{\prime}}
$$

A similar expression is valid for the homoskedastic RML-dif estimator, except that in that case the parameter vector is redefined as $\gamma=\left(\alpha, \sigma^{2}, \sigma_{\eta \dagger}^{2}\right)^{\prime}$.

\section{B.2. Asymptotic Variance of the RML-lev Estimator}

In order to exploit the previous result for the differences, we express the covariance structure corresponding to the levels using the transformation:

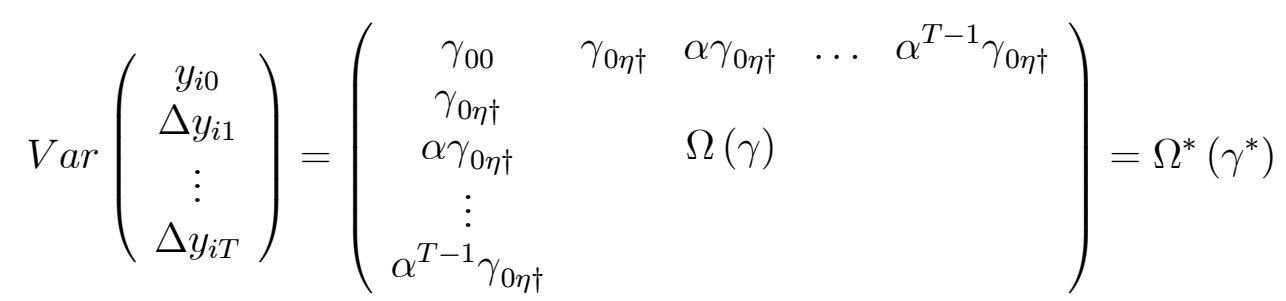

where $\gamma_{00}=\operatorname{Var}\left(y_{i 0}\right), \gamma_{0 \eta \dagger}=\operatorname{Cov}\left(y_{i 0}, \eta_{i}^{\dagger}\right)$, and $\gamma^{*}=\left(\alpha, \sigma_{1}^{2}, \ldots, \sigma_{T}^{2}, \sigma_{\eta \dagger}^{2}, \gamma_{0 \eta \dagger}, \gamma_{00}\right)^{\prime}$.

Arguing as in the previous case, the marginal MLE for the data in levels can be written as

$$
\left(\widehat{\alpha}_{L}, \widetilde{\sigma}_{1}^{2}, \ldots, \widetilde{\sigma}_{T}^{2}, \widetilde{\sigma}_{\eta \dagger}^{2}, \widetilde{\gamma}_{0 \eta \dagger}, \widetilde{\gamma}_{00}\right)=
$$

$$
\arg \min \left[\ln \operatorname{det} \Omega^{*}\left(\gamma^{*}\right)+\frac{1}{N} \sum_{i=1}^{N}\left(y_{i 0}, \Delta y_{i 1}, \ldots, \Delta y_{i T}\right) \Omega^{*-1}\left(\gamma^{*}\right)\left(\begin{array}{c}
y_{i 0} \\
\Delta y_{i 1} \\
\vdots \\
\Delta y_{i T}
\end{array}\right)\right] \text {. }
$$

\footnotetext{
${ }^{15}$ See for example Arellano (2003, p. 72).
} 
Thus, under normality the asymptotic variance matrix of $\left(\widehat{\alpha}_{L}, \widetilde{\sigma}_{1}^{2}, \ldots, \widetilde{\sigma}_{T}^{2}, \widetilde{\sigma}_{\eta \dagger}^{2}, \widetilde{\gamma}_{0 \eta \dagger}, \widetilde{\gamma}_{00}\right)$ is given by

$$
2\left\{H^{*}\left(\gamma^{*}\right)^{\prime} \mathcal{D}^{* \prime}\left[\Omega^{*-1}\left(\gamma^{*}\right) \otimes \Omega^{*-1}\left(\gamma^{*}\right)\right] \mathcal{D}^{*} H^{*}\left(\gamma^{*}\right)\right\}^{-1}
$$

where

$$
H^{*}\left(\gamma^{*}\right)=\frac{\partial \operatorname{vech}\left[\Omega^{*}\left(\gamma^{*}\right)\right]}{\partial \gamma^{* \prime}}
$$

and $\mathcal{D}^{*}$ is the selection matrix

$$
\mathcal{D}^{*}=\frac{\partial v e c \Omega^{*}}{\partial\left(\operatorname{vech} \Omega^{*}\right)^{\prime}}
$$

Note that in this parameterization, under stationary initial conditions, $\gamma_{00}$ remains a free parameter (which determines $\sigma_{\eta}^{2}$ ) given by

$$
\gamma_{00}=\frac{\sigma_{\eta}^{2}}{(1-\alpha)^{2}}+\bar{\sigma}_{0}^{2}
$$

and

$$
\begin{aligned}
\gamma_{0 \eta \dagger} & \equiv \operatorname{Cov}\left(y_{i 0}, \eta_{i}^{\dagger}\right)=-(1-\alpha) \bar{\sigma}_{0}^{2} \\
\sigma_{\eta \dagger}^{2} & \equiv \operatorname{Var}\left(\eta_{i}^{\dagger}\right)=(1-\alpha)^{2} \bar{\sigma}_{0}^{2}
\end{aligned}
$$

so that the restriction under mean stationarity is $\gamma_{0 \eta \dagger} / \sigma_{\eta \dagger}^{2}=-1 /(1-\alpha)$. Homoskedasticity further restricts these coefficients to satisfy $\bar{\sigma}_{0}^{2}=\sigma^{2} /\left(1-\alpha^{2}\right)$.

\section{B.3. Asymptotic Variance of the Homoskedastic BCS Estimator}

Because of the incidental parameters problem, the ML estimates of $\alpha$ and $\sigma^{2}$ estimated jointly with the effects are inconsistent for fixed $T$. However, as noted by Lancaster (2002), we can obtain score adjusted estimators that are consistent in view of the moment relationships:

$$
\begin{aligned}
& E\left(x_{i}^{* \prime} v_{i}^{*}\right)=-\sigma^{2} h_{T}(\alpha) \\
& E\left(v_{i}^{* \prime} v_{i}^{*}\right)=(T-1) \sigma^{2}
\end{aligned}
$$

where $x_{i}^{*}$ and $v_{i}^{*}$ denote orthogonal deviations of the original variables.

By substituting the second equation we can eliminate $\sigma^{2}$ and get

$$
E\left[\psi_{i}(\alpha)\right]=0
$$

where

$$
\psi_{i}(\alpha)=x_{i}^{* \prime} v_{i}^{*}+v_{i}^{* \prime} v_{i}^{*} \frac{h_{T}(\alpha)}{(T-1)}
$$


Under suitable regularity conditions, if there is a consistent root of the equation $\sum_{i=1}^{N} \psi_{i}(a)=0,{ }^{16}$ its asymptotic variance is given by

$$
v_{\alpha}=\frac{v}{d^{2}}
$$

where

$$
v=E\left[\psi_{i}^{2}(\alpha)\right]
$$

and

$$
d=E\left[\frac{\partial \psi_{i}(\alpha)}{\partial \alpha}\right]
$$

Because of

$$
\frac{\partial \psi_{i}(\alpha)}{\partial \alpha}=-x_{i}^{* \prime} x_{i}^{*}-2 x_{i}^{* \prime} v_{i}^{*} \frac{h_{T}(\alpha)}{(T-1)}+\frac{v_{i}^{* \prime} v_{i}^{*}}{(T-1)} h_{T}^{\prime}(\alpha),
$$

we have

$$
d=-E\left(x_{i}^{* \prime} x_{i}^{*}\right)+2 \sigma^{2} \frac{h_{T}^{2}}{(T-1)}+\sigma^{2} h_{T}^{\prime}
$$

where we are using $h_{T}$ and $h_{T}^{\prime}$ for shortness.

Similarly,

$$
v=E\left[\left(x_{i}^{* \prime} v_{i}^{*}\right)^{2}\right]+E\left[\left(v_{i}^{* \prime} v_{i}^{*}\right)^{2}\right] \frac{h_{T}^{2}}{(T-1)^{2}}+2 E\left[\left(x_{i}^{* \prime} v_{i}^{*}\right)\left(v_{i}^{* \prime} v_{i}^{*}\right)\right] \frac{h_{T}}{(T-1)} .
$$

The availability of expression (B.1) allows us to calculate the term $E\left(x_{i}^{* \prime} x_{i}^{*}\right)$ that appears in (B.6) as follows

$$
E\left(x_{i}^{* \prime} x_{i}^{*}\right)=E\left(x_{i}^{\prime} D^{\prime}\left(D D^{\prime}\right)^{-1} D x_{i}\right)=\operatorname{tr}\left[\left(D D^{\prime}\right)^{-1} \Omega_{\Delta 11}\right]
$$

where $\Omega_{\Delta 11}=E\left(D x_{i} x_{i}^{\prime} D^{\prime}\right)$ is the $(T-1) \times(T-1)$ north-west submatrix of $\Omega(\gamma)$ under homoskedasticity.

Next, under normality and homoskedasticity we have

$$
\begin{gathered}
E\left[\left(x_{i}^{* \prime} v_{i}^{*}\right)^{2}\right]=\sigma^{4} h_{T}^{2}+\sigma^{2} E\left(x_{i}^{* \prime} x_{i}^{*}\right)+\sigma^{4} \operatorname{tr}\left(Q C_{T} Q C_{T}\right) \\
E\left[\left(v_{i}^{* \prime} v_{i}^{*}\right)^{2}\right]=\sigma^{4}(T+1)(T-1) \\
E\left[\left(x_{i}^{* \prime} v_{i}^{*}\right)\left(v_{i}^{* \prime} v_{i}^{*}\right)\right]=-\sigma^{4} h_{T}(T+1)
\end{gathered}
$$

where $Q=I_{T}-\iota \iota^{\prime} / T$ and $C_{T}$ is such that $E\left(x_{i} v_{i}^{\prime}\right)=\sigma^{2} C_{T}$.

\footnotetext{
${ }^{16}$ A formal proof of consistency is given in Lancaster (2002), Theorem A1.
} 
Thus,

$$
v=\sigma^{4} h_{T}^{2}+\sigma^{2} E\left(x_{i}^{* \prime} x_{i}^{*}\right)+\sigma^{4} \operatorname{tr}\left(Q C_{T} Q C_{T}\right)-\sigma^{4} h_{T}^{2}\left(\frac{T+1}{T-1}\right)
$$

or

$$
v=\sigma^{2} E\left(x_{i}^{* \prime} x_{i}^{*}\right)+\sigma^{4} \operatorname{tr}\left(Q C_{T} Q C_{T}\right)-\frac{2}{(T-1)} \sigma^{4} h_{T}^{2} .
$$

To get the results (B.9)-(B.11) we have used the following intermediate formulae for moments of quadratic forms in normal variables:

$$
\begin{aligned}
E\left[\left(x_{i}^{* \prime} v_{i}^{*}\right)^{2}\right] & =\left[E\left(x_{i}^{* \prime} v_{i}^{*}\right)\right]^{2}+\operatorname{tr}\left[E\left(x_{i}^{*} x_{i}^{* \prime}\right) E\left(v_{i}^{*} v_{i}^{* \prime}\right)\right]+\operatorname{tr}\left[E\left(x_{i}^{*} v_{i}^{* \prime}\right) E\left(x_{i}^{*} v_{i}^{* \prime}\right)\right] \\
E\left[\left(v_{i}^{* \prime} v_{i}^{*}\right)^{2}\right] & =\operatorname{tr}^{2}\left[E\left(v_{i}^{*} v_{i}^{* \prime}\right)\right]+2 \operatorname{tr}\left[E\left(v_{i}^{*} v_{i}^{* \prime}\right) E\left(v_{i}^{*} v_{i}^{* \prime}\right)\right]=(T-1)^{2} \sigma^{4}+2 \sigma^{4}(T-1) \\
E\left[\left(x_{i}^{* \prime} v_{i}^{*}\right)\left(v_{i}^{* \prime} v_{i}^{*}\right)\right] & =E\left(x_{i}^{* \prime} v_{i}^{*}\right) E\left(v_{i}^{* \prime} v_{i}^{*}\right)+2 \operatorname{tr}\left[E\left(x_{i}^{*} v_{i}^{* \prime}\right) E\left(v_{i}^{*} v_{i}^{* \prime}\right)\right] \\
& =-\sigma^{4} h_{T}(T-1)-2 \sigma^{4} h_{T} .
\end{aligned}
$$

\section{B.4. Asymptotic Variance of the Heteroskedastic BCS Estimator}

The $i$-th unit $\log$ likelihood conditioned on the MLE of $\eta_{i}$ and $y_{i 0}$ is given by

$$
\ell_{i}=-\frac{1}{2} \ln \operatorname{det}\left(D \Lambda D^{\prime}\right)-\frac{1}{2} v_{i}^{\prime} D^{\prime}\left(D \Lambda D^{\prime}\right)^{-1} D v_{i}
$$

where $D$ is the $(T-1) \times T$ first-difference matrix operator and $\Lambda=\operatorname{diag}\left(\sigma_{1}^{2}, \ldots, \sigma_{T}^{2}\right)$. Also, let $d_{t}$ be the $t$-th column of $D$, so that $D \Lambda D^{\prime}=\sum_{t=1}^{T} \sigma_{t}^{2} d_{t} d_{t}^{\prime}$.

Using for shortness the notation $\Omega=D \Lambda D^{\prime}$, the first and second derivatives of $\ell_{i}$ with respect to $\alpha$ and $\sigma_{t}^{2}$ are given by ${ }^{17}$

$$
\begin{gathered}
\frac{\partial \ell_{i}}{\partial \alpha}=x_{i}^{\prime} D^{\prime} \Omega^{-1} D v_{i} \\
\frac{\partial \ell_{i}}{\partial \sigma_{t}^{2}}=\frac{1}{2} d_{t}^{\prime} \Omega^{-1}\left(D v_{i} v_{i}^{\prime} D^{\prime}-\Omega\right) \Omega^{-1} d_{t} \quad(t=1, \ldots, T) \\
\frac{\partial^{2} \ell_{i}}{\partial \alpha^{2}}=-x_{i}^{\prime} D^{\prime} \Omega^{-1} D x_{i} \\
\frac{\partial^{2} \ell_{i}}{\partial \sigma_{t}^{2} \partial \alpha}=-d_{t}^{\prime} \Omega^{-1} D x_{i} v_{i}^{\prime} D^{\prime} \Omega^{-1} d_{t} \quad(t=1, \ldots, T)
\end{gathered}
$$

\footnotetext{
${ }^{17}$ Note that

$$
\frac{\partial d_{t}^{\prime} \Omega^{-1} d_{t}}{\partial \sigma_{s}^{2}}=-\left(d_{t}^{\prime} \Omega^{-1} d_{s}\right)^{2}
$$

and

$$
\frac{\partial}{\partial \sigma_{s}^{2}} d_{t}^{\prime} \Omega^{-1}\left(D v_{i} v_{i}^{\prime} D^{\prime}\right) \Omega^{-1} d_{t}=-2\left(d_{t}^{\prime} \Omega^{-1} d_{s}\right)\left(d_{s}^{\prime} \Omega^{-1} D v_{i} v_{i}^{\prime} D^{\prime} \Omega^{-1} d_{t}\right) .
$$
}




$$
\frac{\partial^{2} \ell_{i}}{\partial \sigma_{t}^{2} \partial \sigma_{s}^{2}}=-\left(d_{t}^{\prime} \Omega^{-1} d_{s}\right)\left(d_{t}^{\prime} \Omega^{-1} D v_{i} v_{i}^{\prime} D^{\prime} \Omega^{-1} d_{s}\right)+\frac{1}{2}\left(d_{t}^{\prime} \Omega^{-1} d_{s}\right)^{2} .
$$

Let $\ell_{1 i}=\partial \ell_{i} / \partial \alpha, \ell_{2 i t}=\partial \ell_{i} / \partial \sigma_{t}^{2}, \ell_{11 i}=\partial^{2} \ell_{i} / \partial \alpha^{2}$, etc., $\gamma=\left(\alpha, \sigma_{1}^{2}, \ldots, \sigma_{T}^{2}\right)^{\prime}$, and $h=-E\left(\ell_{1 i}\right), h_{1}=\partial h / \partial \alpha, h_{2 t}=\partial h / \partial \sigma_{t}^{2}$. BCS is the GMM estimator based on the moments

$$
\psi_{i}=\left(\begin{array}{c}
\psi_{1 i} \\
\psi_{2 i}
\end{array}\right)=\left(\begin{array}{c}
\ell_{1 i}+h \\
\ell_{2 i}
\end{array}\right)
$$

whose asymptotic variance is

$$
V_{B C S}=\left(D^{\prime} V^{-1} D\right)^{-1}
$$

where

$$
D=E\left(\frac{\partial \psi_{i}}{\partial \gamma^{\prime}}\right)=E\left(\begin{array}{cc}
\ell_{11 i} & \ell_{12 i} \\
\ell_{21 i} & \ell_{22 i}
\end{array}\right)+\left(\begin{array}{cc}
h_{1} & h_{2}^{\prime} \\
0 & 0
\end{array}\right)
$$

and

$$
V=E\left(\psi_{i} \psi_{i}^{\prime}\right)=E\left(\begin{array}{cc}
\ell_{1 i}^{2} & \ell_{1 i} \ell_{2 i}^{\prime} \\
\ell_{2 i} \ell_{1 i} & \ell_{2 i} \ell_{2 i}^{\prime}
\end{array}\right)-\left(\begin{array}{cc}
h^{2} & 0 \\
0 & 0
\end{array}\right) .
$$

Letting $\Omega_{\Delta 11}=E\left(D x_{i} x_{i}^{\prime} D^{\prime}\right)$, the expected second derivatives are

$$
\begin{gathered}
E\left(\ell_{11 i}\right) \equiv E\left(\frac{\partial^{2} \ell_{i}}{\partial \alpha^{2}}\right)=-\operatorname{tr}\left(\Omega^{-1} \Omega_{\Delta 11}\right) \\
E\left(\ell_{21 i t}\right) \equiv E\left(\frac{\partial^{2} \ell_{i}}{\partial \sigma_{t}^{2} \partial \alpha}\right)=-d_{t}^{\prime} \Omega^{-1} D C_{1} \Lambda D^{\prime} \Omega^{-1} d_{t} \\
E\left(\ell_{22 i t s}\right) \equiv E\left(\frac{\partial^{2} \ell_{i}}{\partial \sigma_{t}^{2} \partial \sigma_{s}^{2}}\right)=-\frac{1}{2}\left(d_{t}^{\prime} \Omega^{-1} d_{s}\right)^{2}
\end{gathered}
$$

where $E\left(x_{i} v_{i}^{\prime}\right)=C_{1} \Lambda$, and

$$
C_{1}=\left(\begin{array}{cc}
0 & 0 \\
B_{T-1}^{-1} & 0
\end{array}\right)
$$

Finally, the outer product terms are given by

$$
\begin{aligned}
E\left(\ell_{1 i}^{2}\right) & =E\left[\left(x_{i}^{\prime} D^{\prime} \Omega^{-1} D v_{i}\right)^{2}\right] \\
E\left(\ell_{2 i t} \ell_{1 i}\right) & =\frac{1}{2} E\left[\left(d_{t}^{\prime} \Omega^{-1} D v_{i}\right)^{2}\left(x_{i}^{\prime} D^{\prime} \Omega^{-1} D v_{i}\right)\right]+\frac{1}{2}\left(d_{t}^{\prime} \Omega^{-1} d_{t}\right) h \\
E\left(\ell_{2 i t} \ell_{2 i s}\right) & =\frac{1}{4} E\left[\left(d_{t}^{\prime} \Omega^{-1} D v_{i}\right)^{2}\left(d_{s}^{\prime} \Omega^{-1} D v_{i}\right)^{2}\right]-\frac{1}{4}\left(d_{t}^{\prime} \Omega^{-1} d_{t}\right)\left(d_{s}^{\prime} \Omega^{-1} d_{s}\right) .
\end{aligned}
$$


Under normality:

$$
\begin{aligned}
E\left(\ell_{1 i}^{2}\right) & =\operatorname{tr}\left(\Omega^{-1} \Omega_{\Delta 11}\right)+\operatorname{tr}\left(D^{\prime} \Omega^{-1} D C_{1} \Lambda D^{\prime} \Omega^{-1} D C_{1} \Lambda\right)+h^{2} \\
E\left(\ell_{2 i t} \ell_{1 i}\right) & =d_{t}^{\prime} \Omega^{-1} D C_{1} \Lambda D^{\prime} \Omega^{-1} d_{t} \\
E\left(\ell_{2 i t} \ell_{2 i s}\right) & =\frac{1}{2}\left(d_{t}^{\prime} \Omega^{-1} d_{s}\right)^{2}
\end{aligned}
$$

Proof: Note that under normality:

$$
\begin{aligned}
E\left[\left(d_{t}^{\prime} \Omega^{-1} D v_{i}\right)^{2}\left(d_{s}^{\prime} \Omega^{-1} D v_{i}\right)^{2}\right]= & E\left[\left(d_{t}^{\prime} \Omega^{-1} D v_{i}\right)^{2}\right] E\left[\left(d_{s}^{\prime} \Omega^{-1} D v_{i}\right)^{2}\right] \\
& +2\left\{E\left[\left(d_{t}^{\prime} \Omega^{-1} D v_{i}\right)\left(d_{s}^{\prime} \Omega^{-1} D v_{i}\right)\right]\right\}^{2} \\
= & \left(d_{t}^{\prime} \Omega^{-1} d_{t}\right)\left(d_{s}^{\prime} \Omega^{-1} d_{s}\right)+2\left(d_{t}^{\prime} \Omega^{-1} d_{s}\right)^{2}
\end{aligned}
$$

which proves (B.18) and also shows that $E\left(\ell_{2 i t} \ell_{2 i s}\right)=-E\left(\ell_{22 i t s}\right)$.

To prove (B.16), let $v_{i}^{*}=\Omega^{-1 / 2} D v_{i}, x_{i}^{*}=\Omega^{-1 / 2} D x_{i}$ and note that

$$
\begin{aligned}
E\left(\ell_{1 i}^{2}\right) & =E\left[\left(x_{i}^{* \prime} v_{i}^{*}\right)^{2}\right] \\
& =\left[E\left(x_{i}^{* \prime} v_{i}^{*}\right)\right]^{2}+\operatorname{tr}\left[E\left(x_{i}^{*} x_{i}^{* \prime}\right) E\left(v_{i}^{*} v_{i}^{* \prime}\right)\right]+\operatorname{tr}\left[E\left(x_{i}^{*} v_{i}^{* \prime}\right) E\left(x_{i}^{*} v_{i}^{* \prime}\right)\right] \\
& =h^{2}+\operatorname{tr}\left(\Omega^{-1} \Omega_{\Delta 11}\right)+\operatorname{tr}\left(\Omega^{-1} D C_{1} \Lambda D^{\prime} \Omega^{-1} D C_{1} \Lambda D^{\prime}\right) .
\end{aligned}
$$

Finally, (B.17) can be proved as follows:

$$
\begin{aligned}
& E\left[d_{t}^{\prime} \Omega^{-1} D v_{i} v_{i}^{\prime} D^{\prime} \Omega^{-1} d_{t}\left(x_{i}^{\prime} D^{\prime} \Omega^{-1} D v_{i}\right)\right]=E\left[d_{t}^{\prime} \Omega^{-1 / 2 \prime} v_{i}^{*} v_{i}^{* \prime} \Omega^{-1 / 2} d_{t}\left(x_{i}^{* \prime} v_{i}^{*}\right)\right] \\
& =E\left(d_{t}^{\prime} \Omega^{-1 / 2 \prime} v_{i}^{*} v_{i}^{* \prime} \Omega^{-1 / 2} d_{t}\right) E\left(x_{i}^{* \prime} v_{i}^{*}\right)+2 E\left(d_{t}^{\prime} \Omega^{-1 / 2 \prime} v_{i}^{*} x_{i}^{* \prime}\right) E\left(v_{i}^{*} v_{i}^{* \prime} \Omega^{-1 / 2} d_{t}\right) \\
& =-\left(d_{t}^{\prime} \Omega^{-1} d_{t}\right) h+2\left(d_{t}^{\prime} \Omega^{-1} D C_{1} \Lambda D^{\prime} \Omega^{-1} d_{t}\right)
\end{aligned}
$$

To see this, letting $\widetilde{v}_{i t}=d_{t}^{\prime} \Omega^{-1 / 2 \prime} v_{i}^{*}$, note that

$$
\begin{gathered}
E\left[d_{t}^{\prime} \Omega^{-1 / 2 \prime} v_{i}^{*} v_{i}^{* \prime} \Omega^{-1 / 2} d_{t}\left(x_{i}^{* \prime} v_{i}^{*}\right)\right]=\sum_{s} E\left(\widetilde{v}_{i t}^{2} x_{i s}^{*} v_{i s}^{*}\right) \\
=\sum_{s} E\left(\widetilde{v}_{i t}^{2}\right) E\left(x_{i s}^{*} v_{i s}^{*}\right)+2 \sum_{s} E\left(\widetilde{v}_{i t} x_{i s}^{*}\right) E\left(\widetilde{v}_{i t} v_{i s}^{*}\right) \\
=E\left(d_{t}^{\prime} \Omega^{-1 / 2 \prime} v_{i}^{*} v_{i}^{* \prime} \Omega^{-1 / 2} d_{t}\right) E\left(x_{i}^{* \prime} v_{i}^{*}\right)+2 E\left(d_{t}^{\prime} \Omega^{-1 / 2 \prime} v_{i}^{*} x_{i}^{* \prime}\right) E\left(v_{i}^{*} v_{i}^{* \prime} \Omega^{-1 / 2} d_{t}\right) .
\end{gathered}
$$

Thus, the information equality $E\left(\ell_{2 i t} \ell_{1 i}\right)=-E\left(\ell_{21 i t}\right)$ also holds. 


\section{Modified Conditional ML Score Interpretation of BCS}

For the heteroskedastic $\mathrm{AR}(1)$ model we saw that $\mathrm{BCS}$ can be given a modified conditional likelihood interpretation when the weights $\varphi$ are known. More generally, we show here that for a heteroskedastic $\operatorname{AR}(p)$ model with unknown weights, the BCS estimating equations coincide with the modified score vector discussed in Arellano (2003b), which is first reviewed for convenience.

The Modified CML Score Let $\ell_{i}\left(\beta, \eta_{i}\right)$ be an individual log-likelihood conditioned on $z_{i}$, and let $d_{\beta i}\left(\beta, \eta_{i}\right), d_{\eta i}\left(\beta, \eta_{i}\right), d_{\eta \eta i}\left(\beta, \eta_{i}\right)$ and $d_{\beta \eta i}\left(\beta, \eta_{i}\right)$ be first and second partial derivatives. The first argument is a vector common parameter $\beta$ and $\eta_{i}$ is a scalar individual effect. Let $\ell_{i}\left(\beta, \hat{\eta}_{i}(\beta)\right)$ be the concentrated log likelihood, so that $d_{\beta i}\left(\beta, \widehat{\eta}_{i}(\beta)\right)$ is the concentrated score.

The modified score discussed in Arellano (2003b) is given by

$$
d_{M i}(\beta)=d_{\beta i}\left(\beta, \widehat{\eta}_{i}(\beta)\right)-\frac{1}{2} \frac{\partial}{\partial \beta} \ln \left[-d_{\eta \eta i}\left(\beta, \widehat{\eta}_{i}(\beta)\right)\right]+q_{\eta i}\left(\beta, \widehat{\eta}_{i}(\beta)\right)
$$

where

$$
\begin{aligned}
q_{\eta i}\left(\beta, \eta_{i}\right) & =\frac{\partial}{\partial \eta_{i}} q_{i}\left(\beta, \eta_{i}\right) \\
q_{i}\left(\beta, \eta_{i}\right) & =\frac{\kappa_{\beta \eta i}\left(\beta, \eta_{i}\right)}{\kappa_{\eta \eta i}\left(\beta, \eta_{i}\right)}
\end{aligned}
$$

and

$$
\begin{aligned}
\kappa_{\beta \eta i}\left(\beta_{0}, \eta_{i}\right) & =E\left[\frac{1}{T} d_{\beta \eta i}\left(\beta_{0}, \eta_{i}\right) \mid x_{i}, \eta_{i}\right] \\
\kappa_{\eta \eta i}\left(\beta_{0}, \eta_{i}\right) & =E\left[\frac{1}{T} d_{\eta \eta i}\left(\beta_{0}, \eta_{i}\right) \mid x_{i}, \eta_{i}\right] .
\end{aligned}
$$

The first modification term provides a "degrees of freedom adjustment", whereas the second corrects for nonorthogonality between $\beta$ and $\eta_{i}$. Note that if $\beta$ and $\eta_{i}$ are information orthogonal $\kappa_{\beta \eta i}\left(\beta, \eta_{i}\right)=0$, so that $q_{\eta i}\left(\beta, \eta_{i}\right)=0$ as well.

If there exists a scalar function $c_{i}\left(\beta, \eta_{i}\right)$ such that

$$
\frac{\partial}{\partial \beta} c_{i}\left(\beta, \eta_{i}\right)=q_{\eta i}\left(\beta, \eta_{i}\right)
$$

the modified score corresponds to the objective function

$$
\ell_{i}\left(\beta, \widehat{\eta}_{i}(\beta)\right)-\frac{1}{2} \ln \left[-d_{\eta \eta i}\left(\beta, \widehat{\eta}_{i}(\beta)\right)\right]+c_{i}\left(\beta, \eta_{i}\right),
$$

which coincides with the Cox and Reid modified profile likelihood based on an orthogonal reparameterization of the effects. If $c_{i}\left(\beta, \eta_{i}\right)$ does not exist, there is no orthogonal reparameterization but the modified score $d_{M i}(\beta)$ may still achieve bias reduction relative to $d_{\beta i}\left(\beta, \widehat{\eta}_{i}(\beta)\right)$. 

and

Application to $\operatorname{AR}(p)$ models In the $\operatorname{AR}(p)$ model, $\beta=\left(\alpha^{\prime}, \theta^{\prime}\right)^{\prime}, z_{i}=y_{i}^{0}$,

$$
\begin{gathered}
\ell_{i}\left(\beta, \eta_{i}\right)=-.5 \ln \operatorname{det} \Lambda-.5 v_{i}^{\prime} \Lambda^{-1} v_{i}, \quad d_{\eta \eta i}\left(\beta, \eta_{i}\right)=-1 / \omega_{T}, \\
d_{\beta \eta i}\left(\beta, \eta_{i}\right)=-\left(\frac{1}{\omega_{T}} \bar{x}_{1 i}, \ldots, \frac{1}{\omega_{T}} \bar{x}_{p i}, \frac{1}{\sigma_{1}^{4}} v_{i 1}, \ldots, \frac{1}{\sigma_{T}^{4}} v_{i T}\right)^{\prime} .
\end{gathered}
$$

Thus, $\kappa_{\eta \eta i}\left(\beta, \eta_{i}\right)=-1 /\left(T \omega_{T}\right)$,

$$
\begin{aligned}
\kappa_{\beta \eta i}\left(\beta_{0}, \eta_{i}\right) & =E\left[\frac{1}{T} d_{\beta \eta i}\left(\beta_{0}, \eta_{i}\right) \mid y_{i}^{0}, \eta_{i}\right] \\
& =-\frac{1}{T \omega_{T}}\left(E\left(\bar{x}_{1 i} \mid y_{i}^{0}, \eta_{i}\right), \ldots, E\left(\bar{x}_{p i} \mid y_{i}^{0}, \eta_{i}\right), 0, \ldots, 0\right)^{\prime},
\end{aligned}
$$

and

$$
q_{i}\left(\beta, \eta_{i}\right)=\left(\begin{array}{c}
\eta_{i}\left(\varphi^{\prime} C_{1} \iota\right)+\left(\varphi^{\prime} C_{T p}^{1}\right) y_{i}^{0} \\
\vdots \\
\eta_{i}\left(\varphi^{\prime} C_{p} \iota\right)+\left(\varphi^{\prime} C_{T p}^{1}\right) y_{i}^{0} \\
0 \\
\vdots \\
0
\end{array}\right), \quad q_{\eta i}\left(\beta, \eta_{i}\right)=\left(\begin{array}{c}
\varphi^{\prime} C_{1} \iota \\
\vdots \\
\varphi^{\prime} C_{p} \iota \\
0 \\
\vdots \\
0
\end{array}\right)
$$

Therefore, the modified score vector is

$$
d_{M i}(\beta)=d_{\beta i}\left(\beta, \widehat{\eta}_{i}(\beta)\right)+\frac{1}{2} \frac{\partial}{\partial \beta} \ln \omega_{T}+\left(\varphi^{\prime} C_{1} \iota, \ldots, \varphi^{\prime} C_{p} \iota, 0, \ldots, 0\right)^{\prime}
$$

where

$$
d_{\beta i}\left(\beta, \widehat{\eta}_{i}(\beta)\right)=\frac{\partial}{\partial \beta}\left[-\frac{1}{2} \ln \operatorname{det} \Phi-\frac{T}{2} \ln \omega_{T}-\frac{1}{2 \omega_{T}} v_{i}^{\prime}\left(\Phi-\Phi \iota \iota^{\prime} \Phi\right) v_{i}\right],
$$

which shows that BCS can be regarded as the solution to the estimating equations $\sum_{i=1}^{N} d_{M i}(\beta)=0$.

In this case it does not exist a function $c_{i}\left(\beta, \eta_{i}\right)$ such that

$$
(\partial / \partial \beta) c_{i}\left(\beta, \eta_{i}\right)=\left(\varphi^{\prime} C_{1} \iota, \ldots, \varphi^{\prime} C_{p} \iota, 0, \ldots, 0\right)^{\prime} .
$$

This can be easily seen when $p=1$. In that case $h_{T}(\alpha, \varphi)=\partial b_{T}(\alpha, \varphi) / \partial \alpha$ where $b_{T}(\alpha, \varphi)=\sum_{t=1}^{T-1}\left(\varphi_{t+1}+\ldots+\varphi_{T}\right) \alpha^{t} / t$, so that possible solutions for $c_{i}\left(\beta, \eta_{i}\right)$ would be of the form $b_{T}(\alpha, \varphi)+c(\theta)$. However, since $\partial b_{T}(\alpha, \varphi) / \partial \sigma_{t}^{2}$ depends on $\alpha$ and varies with $t,{ }^{18}$ there is no $c(\theta)$ that can make $\partial c_{i}\left(\beta, \eta_{i}\right) / \partial \sigma_{t}^{2}$ equal to zero for any $\alpha$ and $t$ as required.

Thus, in the heteroskedastic $\operatorname{AR}(p)$ setting, despite the lack of existence of an orthogonal transformation, a first-order bias adjustment to the score is an exact correction that removes fully the bias, hence leading to fixed- $T$ consistent estimation.

\footnotetext{
${ }^{18}$ The expression is $\partial b_{T}(\alpha, \varphi) / \partial \sigma_{t}^{2}=-\varphi_{t}^{2}\left[b_{T}(\alpha, \varphi)+\alpha+. .+\alpha^{t-1}\right] / \omega_{T}$.
} 


\section{ARMA Models}

Consider the model

$$
y_{i t}=\alpha_{1} y_{i(t-1)}+\ldots+\alpha_{p} y_{i(t-p)}+\eta_{i}+v_{i t} \quad(t=1, \ldots, T ; i=1, \ldots, N)
$$

where $v_{i t}$ is a moving average error of order $q$.

Following the notation introduced in (A.9), we can write

$$
\left(\begin{array}{cc}
I_{p} & 0 \\
B_{T p} & B_{T}
\end{array}\right)\left(\begin{array}{c}
y_{i}^{0} \\
y_{i}
\end{array}\right)=\left(\begin{array}{c}
y_{i}^{0} \\
\eta_{i} \iota+v_{i}
\end{array}\right) \text {. }
$$

For an $\operatorname{AR}(p)$ process we have

$$
\operatorname{Var}\left(\begin{array}{c}
y_{i}^{0} \\
\eta_{i} \iota+v_{i}
\end{array}\right)=\left(\begin{array}{cc}
\Gamma_{00} & \gamma_{0 \eta^{\prime}}^{\prime} \\
\iota_{T} \gamma_{0 \eta}^{\prime} & \sigma_{\eta}^{2} \iota_{T} \iota_{T}^{\prime}+\Lambda
\end{array}\right)
$$

where $\Lambda=\operatorname{diag}\left(\sigma_{1}^{2}, \ldots, \sigma_{T}^{2}\right)$.

Similarly, for an $\operatorname{ARMA}(p, q)$ process

$$
\operatorname{Var}\left(\begin{array}{c}
y_{i}^{0} \\
\eta_{i} \iota+v_{i}
\end{array}\right)=\left(\begin{array}{ccc}
\Gamma_{00} & \Upsilon_{p q} & \gamma_{0 \eta} \iota_{T-q}^{\prime} \\
\Upsilon_{p q}^{\prime} & \sigma_{\eta}^{2} \iota_{T} \iota_{T}^{\prime}+\Lambda_{\psi} \\
\iota_{T-q} \gamma_{0 \eta}^{\prime} &
\end{array}\right)
$$

If $p \leq q$, the elements of $\Upsilon_{p q}$ are all unrestricted. However, if $p>q$ only the last $q$ rows are unrestricted, and the $(p-q)$ first elements of the columns of $\Upsilon_{p q}$ coincide with those of $\gamma_{0 \eta}$. Moreover, $\Lambda_{\psi}$ is a moving average covariance matrix whose first $q$ subdiagonals contain nonzero elements.

We adopt the following heteroskedastic moving-average specification for the errors in (D.1):

$$
\begin{gathered}
v_{i t}=\sigma_{t} v_{i t}^{\dagger} \\
v_{i t}^{\dagger}=\zeta_{i t}-\psi_{1} \zeta_{i(t-1)}-\ldots-\psi_{q} \zeta_{i(t-q)}
\end{gathered}
$$

where $\zeta_{i t}$ is an $i i d(0,1)$ random error. In this way, we allow for arbitrary time series heteroskedasticity and at the same time specify a stationary serial correlation pattern for $v_{i t}$. Thus,

$$
v_{i}=\Lambda^{1 / 2} \Psi\left(\zeta_{i(1-q)}, \ldots, \zeta_{i T}\right)^{\prime}
$$

and

$$
\Lambda_{\psi}=\Lambda^{1 / 2} \Psi \Psi^{\prime} \Lambda^{1 / 2}
$$

where $\Psi$ is the $T \times(T+q)$ matrix

$$
\Psi=\left(\begin{array}{ccccccccccc}
-\psi_{q} & -\psi_{q-1} & \ldots & -\psi_{1} & 1 & 0 & \ldots & 0 & \ldots & 0 & 0 \\
0 & -\psi_{q} & \ldots & -\psi_{2} & -\psi_{1} & 1 & & 0 & \ldots & 0 & 0 \\
\vdots & \vdots & & & & \ddots & \ddots & \ddots & & \vdots & \vdots \\
0 & 0 & \ldots & 0 & 0 & 0 & \ldots & -\psi_{q} & \ldots & -\psi_{1} & 1
\end{array}\right)
$$


Therefore, the covariance matrix of $y_{i}^{T}=\left(y_{i}^{0 \prime}, y_{i}^{\prime}\right)^{\prime}$ is given by

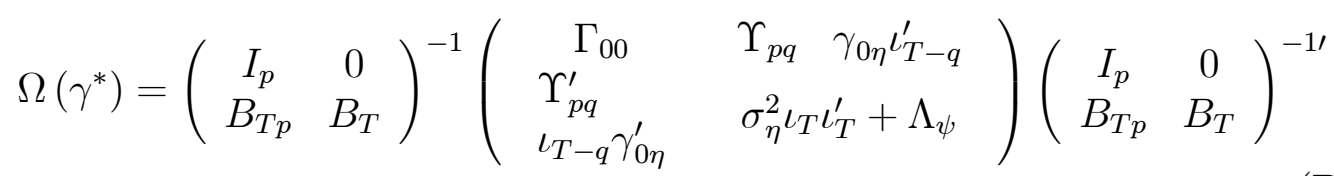

where the parameter vector $\gamma^{*}$ consists of the autoregressive and moving average coefficients, $\gamma_{0 \eta}, \sigma_{\eta}^{2}, \sigma_{1}^{2}, \ldots, \sigma_{T}^{2}$, and the unrestricted elements in $\Gamma_{00}$ and $\Upsilon_{p q}$.

The $\operatorname{ARMA}(p, q)$ log likelihood is given by

$$
L_{R S}=-\frac{N}{2} \ln \operatorname{det} \Omega\left(\gamma^{*}\right)-\frac{1}{2} \sum_{i=1}^{N} y_{i}^{T \prime} \Omega\left(\gamma^{*}\right)^{-1} y_{i}^{T} .
$$

Noting that

$$
\operatorname{det}\left(\begin{array}{cc}
I_{p} & 0 \\
B_{T p} & B_{T}
\end{array}\right)=1
$$

and letting $u_{i}=\eta_{i} \iota+v_{i}, \Omega_{11}=\sigma_{\eta}^{2} \iota \iota^{\prime}+\Lambda_{\psi}, \Gamma_{01}=\left(\begin{array}{ll}\Upsilon_{p q} & \gamma_{0 \eta} \iota_{T-q}^{\prime}\end{array}\right)$, and

where

$$
\left(\begin{array}{ll}
\Gamma_{00} & \Gamma_{01} \\
\Gamma_{01}^{\prime} & \Omega_{11}
\end{array}\right)^{-1}=\left(\begin{array}{cc}
\Gamma^{00} & \Gamma^{01} \\
\Gamma^{01 \prime} & \Omega^{11}
\end{array}\right)
$$

$$
\begin{gathered}
\Omega_{11}^{-1}=\Omega^{11}-\Gamma^{01 \prime}\left(\Gamma^{00}\right)^{-1} \Gamma^{01} \\
\operatorname{det} \Omega\left(\gamma^{*}\right)=\left(\operatorname{det} \Omega_{11}\right) /\left(\operatorname{det} \Gamma^{00}\right),
\end{gathered}
$$

we have

$$
\begin{aligned}
y_{i}^{T \prime} \Omega\left(\gamma^{*}\right)^{-1} y_{i}^{T} & =\left(y_{i}^{0 \prime}, u_{i}^{\prime}\right)\left(\begin{array}{cc}
\Gamma^{00} & \Gamma^{01} \\
\Gamma^{01 \prime} & \Omega^{11}
\end{array}\right)\left(\begin{array}{c}
y_{i}^{0} \\
u_{i}
\end{array}\right) \\
& =u_{i}^{\prime} \Omega_{11}^{-1} u_{i}+\left(y_{i}^{0}+\left(\Gamma^{00}\right)^{-1} \Gamma^{01} u_{i}\right)^{\prime} \Gamma^{00}\left(y_{i}^{0}+\left(\Gamma^{00}\right)^{-1} \Gamma^{01} u_{i}\right)
\end{aligned}
$$

Therefore, letting $\Psi_{00}=\left(\Gamma^{00}\right)^{-1}$ and $\Pi_{01}=-\left(\Gamma^{00}\right)^{-1} \Gamma^{01}=\Gamma_{01} \Omega_{11}^{-1}$, we obtain the following expression for $L_{R S}$ :

$$
\begin{aligned}
L_{R S}= & -\frac{N}{2} \ln \operatorname{det} \Omega_{11}-\frac{1}{2} \sum_{i=1}^{N} u_{i}^{\prime} \Omega_{11}^{-1} u_{i} \\
& -\frac{N}{2} \ln \operatorname{det} \Psi_{00}-\frac{1}{2} \sum_{i=1}^{N}\left(y_{i}^{0}-\Pi_{01} u_{i}\right)^{\prime} \Psi_{00}^{-1}\left(y_{i}^{0}-\Pi_{01} u_{i}\right) .
\end{aligned}
$$

Concentrating the likelihood with respect to $\Psi_{00}$ (which is unrestricted), we get

$$
L_{R S}^{*}=-\frac{N}{2} \ln \operatorname{det} \Omega_{11}-\frac{1}{2} \sum_{i=1}^{N} u_{i}^{\prime} \Omega_{11}^{-1} u_{i}-\frac{N}{2} \ln \operatorname{det} \sum_{i=1}^{N}\left(y_{i}^{0}-\Pi_{01} u_{i}\right)\left(y_{i}^{0}-\Pi_{01} u_{i}\right)^{\prime},
$$

which we found computationally very useful. 
Table A1

Sample characteristics: Spanish Data, 1994-1999

\begin{tabular}{llll}
\multicolumn{4}{c}{$N=632, T^{0}=6$} \\
\hline & Mean & Min & Max \\
\hline age & 43.5 & 23 & 65 \\
tenure (years of exp in the job) & 13.4 & 0 & 20 \\
real labor income (euros) & 13296.8 & 3529.1 & 72825.8 \\
real capital income (euros) & 276.6 & 0 & 27761.8 \\
\% less than sec educ & 28.3 & & \\
\% secondary educ & 46.3 & & \\
\% university educ & 25.4 & & \\
\% industry & 37.0 & & \\
\% service & 63.0 & & \\
\% private sector & 65.0 & & \\
\hline
\end{tabular}

Table A2

Regression results first-step

Dependent variable: log of real labor income

Spanish Data, 1994-1999

\begin{tabular}{lcc}
\hline & Coefficient & t-ratio \\
\hline constant & 7.269 & 54.98 \\
age & 0.076 & 12.79 \\
age2 & -0.001 & -11.47 \\
sec educ & 0.267 & 19.98 \\
univ educ & 0.717 & 46.48 \\
private sector & 0.073 & 5.73 \\
services & -0.006 & -0.50 \\
d94 & -0.040 & -2.15 \\
d95 & -0.051 & -2.79 \\
d96 & -0.054 & -2.95 \\
d97 & -0.049 & -2.68 \\
d98 & -0.027 & -1.50 \\
\hline
\end{tabular}

